\title{
Weyl's Law: Spectral Properties of the Laplacian in Mathematics and Physics
}

Wolfgang Arendt, Robin Nittka, Wolfgang Peter, ${ }^{1)}$ Frank Steiner

\section{1 \\ Introduction}

Weyl's law is in its simplest version a statement on the asymptotic growth of the eigenvalues of the Laplacian on bounded domains with Dirichlet and Neumann boundary conditions. In the typical applications in physics one deals either with the Helmholtz wave equation describing the vibrations of a string, a membrane (drum), a mass of air in a concert hall, the heat radiation from a body in thermal equilibrium, the fluctuations of the gravitational field in cosmology, or the Schrödinger equation of a quantum system which may be a simple quantum billiard, an atom, a molecule or a compound nucleus. While Weyl's seminal work was provoked by the famous black body radiation problem, i.e. an electromagnetic cavity problem, in particular by a conjecture put forward independently by Sommerfeld and Lorentz in 1910, Weyl's law has its roots in music and, respectively, acoustics. Already in 1877, Lord Rayleigh had, in his famous book, "The Theory of Sound" treated the overtones of a violin or piano string and the natural notes of an organ pipe or the air contained within a room. For a room of cubical shape he derived the correct asymptotic behavior for the overtones. The trick used by Rayleigh to count the vibrational modes was to reduce the problem to a three-dimensional lattice-point problem from which he could derive that the number of overtones with frequency between $v$ and $v+\mathrm{d} v$ grows at high frequencies, $v \rightarrow \infty$, asymptotically as $V \cdot v^{3}$ (Weyl's law!), where $V$ is the volume of the room or analogously of an organ pipe. In 1900, Rayleigh realized that the same formula can be applied to a physically completely different, but mathematically equivalent problem: the heat radiation from a body in thermal equilibrium with radiation, the importance of which had been pointed out already in 1859 by Kirchhoff. The amount of energy emitted by a body is determined by the high-frequency spectrum of standing electromagnetic waves and that spectrum should be essentially the same as for the high overtones of an organ pipe, say.

1) Corresponding author.

Mathematical Analysis of Evolution, Information, and Complexity.

Edited by Wolfgang Arendt and Wolfgang P. Schleich

Copyright (C) 2009 WILEY-VCH Verlag GmbH \& Co. KGaA, Weinheim

ISBN: 978-3-527-40830-6 
In the crucial black body radiation experiments carried out in the 1890 s, which led Planck, in 1900, to the famous radiation law named after him and to the discovery of quantum theory, one measures the energy density emitted rather than the energy itself, i.e. the energy divided by the volume $V$. Thus it follows from Rayleigh's asymptotic result $V \cdot v^{3}$, derived for a cubical geometry, that the volume factor is canceled if one considers the energy density, in accordance with the expectations using physical arguments and, very importantly, in complete agreement with the experimental findings. It was realized, however, and emphasized by Sommerfeld and Lorentz in 1910 that there arises the mathematical problem to prove that the number of sufficiently high overtones which lie between $v$ and $v+\mathrm{d} v$ is independent of the shape of the enclosure and is simply proportional to its volume. It was a great achievement when Weyl proved in 1911 that, by applying the FredholmHilbert theory of integral equations, the Sommerfeld-Lorentz conjecture holds! From then on, Weyl himself and many other mathematicians and physicists have studied and generalized Weyl's law by deriving corrections or even complete expressions for the remainder term.

The Weyl asymptotics as discussed above in the three-dimensional case is particularly striking if considered as an inverse spectral problem, which became quite popular after Kac's talk in 1966 entitled "Can one hear the shape of a drum?".

Subsequently, several partially affirmative answers to this question have been given. But, on the other hand, a particularly striking counterexample by Gordon, Webb and Wolpert from 1992 shows that not all geometric information about the domain is contained in the spectrum.

This chapter is organized as follows. In Section 1.2 we give a historical account of Weyl's law. The following two chapters are devoted to Weyl's law with remainder term and the statistical behavior of the latter using trace formulae. We discuss the Laplacian on the torus in Section 1.3 and the Laplace-Beltrami operator on Riemann surfaces in Section 1.4. Then two generalizations of Weyl's law to Robin boundary conditions and for unbounded quantum billiards are presented in Section 1.5. In Section 1.6 we provide a self-contained proof of Weyl's law for bounded Euclidean domains and Dirichlet boundary conditions; the case Weyl himself treated in his first article on this topic. However, we follow a different, very fruitful, approach based on heat kernels. In Section 1.7 we give an account on what is known today about Kac's question. In particular we show under which precise regularity assumptions one can hear whether a body is a ball.

\section{2}

\section{A Brief History of Weyl's Law}

\subsection{1}

\section{Weyl's Seminal Work in 1911-1915}

In February 1911, David Hilbert presented to a meeting of the Royal Academy of Sciences of Göttingen a short note [1] written by Hermann Weyl. This note contains 
for the first time a rigorous proof of the asymptotic behavior of the eigenvalues $\lambda_{n}$ of the two-dimensional (scalar) Helmholtz wave equation

$$
(\Delta+\lambda) u(x)=0 \quad(x \in \Omega)
$$

satisfying the Dirichlet boundary condition

$$
u(x)=0 \quad(x \in \partial \Omega),
$$

where $\Omega \in \mathbb{R}^{2}$ is an arbitrary bounded domain with area $|\Omega|$, boundary $\partial \Omega$, and

$$
\Delta=\frac{\partial^{2}}{\partial x_{1}^{2}}+\frac{\partial^{2}}{\partial x_{2}^{2}}
$$

denotes the Laplacian on $\Omega$. The "membrane problem" $(1.1,1.2)$ has nontrivial solutions $u$ only for a discrete set of eigenvalues $\left\{\lambda_{n}\right\}_{n \in \mathbb{N}}$. The corresponding eigenfunctions $\left\{u_{n}\right\}_{n \in \mathbb{N}}$ provide an orthonormal basis of $L^{2}(\Omega)$, and we may enumerate the eigenvalues in increasing order $0<\lambda_{1} \leq \lambda_{2} \leq \ldots$

Note that the eigenvalues $\lambda_{n}$ can have different physical interpretations. In the case of a vibrating membrane with clamped edge, where $u$ describes the transversal vibrations of the membrane, one has $\lambda_{n}=k_{n}^{2}$, where $k_{n}=(2 \pi / c) v_{n}$ is the wave number which is proportional to the eigenfrequency $v_{n}$, i.e. to the pure tones which the membrane is capable of producing. The constant $c$ is the sound velocity depending on the physical properties of the membrane, i.e. on the mass density and the tension under which the membrane is held. In the case of quantum mechanics, where $u$ is the wave function having the meaning of a probability amplitude, Equation (1.1) is the time independent Schrödinger equation of a freely moving particle with mass $m$, and $\lambda_{n}=\left(2 m / \hbar^{2}\right) E_{n}$ is proportional to the quantal energy levels $E_{n}$. ( $\hbar$ denotes Planck's constant.)

Since explicit analytical expressions for the eigenvalues are known only for a few membranes with simple shape (for example equilateral triangles, rectangles, circles) and their numerical computation for large $n$ is very difficult for general domains, it is natural to study their asymptotic distribution as $n \rightarrow \infty$. Applying the Fredholm-Hilbert theory of linear integral equations, Weyl proved that

$$
\lim _{n \rightarrow \infty} \frac{n}{\lambda_{n}}=\frac{|\Omega|}{4 \pi}
$$

Defining the counting function $N(\lambda):=\#\left\{\lambda_{n} \leq \lambda\right\}$, (1.3) is equivalent to the asymptotic behavior

$$
N(\lambda)=\frac{|\Omega|}{4 \pi} \lambda+o(\lambda) \quad(\lambda \rightarrow \infty) .
$$

These results are now called Weyl's law. Shortly afterwards, Weyl submitted three papers [2-4] which contain the details of his proof, a generalization of (1.4) to the three-dimensional scalar wave equation $\left(\Omega \subset \mathbb{R}^{3}\right)$,

$$
N(\lambda)=\frac{|\Omega|}{6 \pi^{2}} \lambda^{3 / 2}+o\left(\lambda^{3 / 2}\right) \quad(\lambda \rightarrow \infty)
$$


and the extension to the vector Helmholtz wave equation describing the vibrations of the electric field $E$ in an empty cavity $\Omega$ with perfectly reflecting walls $\partial \Omega$. As we shall discuss in more detail in Sections 1.2.3-1.2.8, it is exactly this electrodynamic cavity problem, studied extensively in those years by theoretical physicists, which was one of the open problems that provoked Weyl to start his seminal work.

The electromagnetic cavity problem requires of the electric field vector boundary conditions which are more involved than the simple boundary condition (1.2). In his first papers $[2,4]$ on this problem, Weyl considered some nonphysical boundary conditions; following a suggestion of Levi-Civita, in his subsequent paper [5] the correct boundary conditions $\boldsymbol{E} \times \boldsymbol{n}=0$ and $\nabla \boldsymbol{E}=0$ on $\partial \Omega$ were taken into account. However, the Gauss law $\nabla E=0$ on $\Omega$ i.e. throughout the interior of the cavity, was still discarded.

In his paper [5] Weyl went even one step further and conjectured the existence of a second asymptotic term

$$
N(\lambda)=\frac{|\Omega|}{4 \pi} \lambda \mp \frac{|\partial \Omega|}{4 \pi} \sqrt{\lambda}+o(\sqrt{\lambda}) \quad(\lambda \rightarrow \infty)
$$

for the two-dimensional problem (1.1), where $|\partial \Omega|$ denotes the length of the circumference of the membrane and the $(-)$ sign refers to the Dirichlet boundary condition (1.2) and the $(+)$ sign to the Neumann boundary condition $(\partial u / \partial n=0$, $x \in \partial \Omega)$, and

$$
N(\lambda)=\frac{|\Omega|}{6 \pi^{2}} \lambda^{3 / 2} \mp \frac{|\partial \Omega|}{16 \pi} \lambda+o(\lambda) \quad(\lambda \rightarrow \infty)
$$

for the three-dimensional case, where $|\partial \Omega|$ now denotes the surface area of $\partial \Omega$. The formulae (1.6) and (1.7) became known as Weyl's conjecture. It was justified (under certain conditions on $\Omega$ ) by Ivrii [6] and Melrose [7] only in 1980.

In 1915, Weyl concluded his work on the asymptotic behavior of eigenvalues with a study [8] of the elastic vibrations $\boldsymbol{u}$ of a homogeneous body with volume $|\Omega|$ which are determined by the solutions of the differential equation

$$
B \Delta \boldsymbol{u}+A \operatorname{grad} \operatorname{div} \boldsymbol{u}+\lambda \boldsymbol{u}=0 .
$$

Here $\lambda$ is related to the frequency $v$ by $\lambda=(2 \pi v)^{2}$, and $A, B$ are positive constants (related to the Lamé coefficients characterizing the elastomechanical properties of the body). Imposing the boundary conditions $\nabla \boldsymbol{u}=0$ and $\boldsymbol{n} \times \boldsymbol{u}=0$ on the boundary $\partial \Omega$ of the body, Weyl proved for arbitrary shapes of the body

$$
N(\lambda)=\frac{|\Omega|}{6 \pi^{2}} F \lambda^{3 / 2}+o\left(\lambda^{3 / 2}\right) \quad(\lambda \rightarrow \infty),
$$

where $F$ is a function of the elastic constants, $F=2 / c_{\mathrm{T}}^{3}+1 / c_{\mathrm{L}}^{3}$ with $c_{\mathrm{T}}=\sqrt{B}$ the transverse and $c_{\mathrm{L}}=\sqrt{A+B}$ the longitudinal sound velocity.

The Weyl formulae (1.3)-(1.7) and (1.9) are very striking since they tell us that the coefficient of the leading asymptotic term is determined only by the area, resp., 
the volume of the domain and is independent of its shape. That is one can "hear" the area of a drum (a membrane, held fixed along its boundary) or the volume of a cavity following Marc Kac's [9] acoustic rephrasing of the problem. We refer to Sections 1.2.8, 1.3.6, 1.3.7 and 1.7 for more details on Kac's problem.

In his papers [1,2], Weyl mentions that his asymptotic formulae "provide in particular the solution to a problem the importance of which has recently been emphasized by Sommerfeld and Lorentz" (here and in the following citations, we employ free translations from the original German).

\subsection{2}

\section{The Conjecture of Sommerfeld (1910)}

In September 1910, Arnold Sommerfeld delivered a talk at the "82. NaturforscherVersammlung" in Königsberg [10]. In this talk he studied the solution of the inhomogeneous differential equation in one, two and three dimensions

$$
\left(\Delta_{\Omega}+\lambda\right) v=f
$$

describing forced vibrations. For this purpose, he introduced the resolvent kernel (called the "Green function")

$$
G_{\Omega}(x, y ; \lambda):=\sum_{m} \frac{u_{m}(x) u_{m}(y)}{\lambda-\lambda_{m}} \quad(x, y \in \Omega)
$$

where $u_{m}(x)$ are the eigenfunctions of (1.1). In addition to the Dirichlet boundary condition (1.2), Sommerfeld also considered Neumann boundary conditions and, "as in the theory of heat conduction", Robin boundary conditions $\left(h_{1} u+h_{2}(\partial u / \partial n)=\right.$ 0 on $\partial \Omega, h_{1}, h_{2}$ constant or arbitrary functions on $\partial \Omega$ ). A formal application of the operator $\left(\Delta_{\Omega}+\lambda\right)$ to $G_{\Omega}(x, y ; \lambda)$ (acting on the first argument $x$ ) gives $\left(\Delta_{\Omega}+\lambda\right) G_{\Omega}(x, \gamma ; \lambda)=\sum_{m} u_{m}(x) u_{m}(\gamma)$, and Sommerfeld remarks that this expression is zero for $x \neq y$, but is infinite for $x=\gamma$. He calls this expression "Zackenfunktion" ("spike function"), the physical interpretation of it is a "unit source" (point source). This is, of course, an early attempt to introduce the Dirac delta distribution, since the above expression is nothing other than the completeness relation of the orthonormal eigenfunctions $u_{m} \in L^{2}(\Omega)$

$$
\sum_{m} u_{m}(x) u_{m}(\gamma)=\delta(x-\gamma)
$$

The solution of the inhomogeneous problem (1.10) then reads

$$
v(x)=\int_{\Omega} G_{\Omega}(x, y ; \lambda) f(\gamma) \mathrm{d} \gamma .
$$

This result is quite remarkable since it allows one to reduce the problem (1.10) of the forced vibrations on $\Omega$ to the problem (1.1) of the free vibrations on the same domain $\Omega$. "As some material is fully characterized by its spectral lines i.e. 
by its free vibrational frequencies, so is also the behavior of a domain for arbitrary vibratory motions completely determined by the spectrum of its free vibrational possibilities." [10]

Sommerfeld [10] then discusses the convergence of the series (1.11): "In the one-dimensional case the series (1.11) is absolutely convergent, in the two- and three-dimensional case only conditionally convergent. In the first case the growth of the denominator $\lambda-\lambda_{m}$ is sufficient for convergence, since $[\ldots]$ the denominator becomes infinite, as $m^{2}$. In the latter cases, will $\lambda_{m, n}$, resp. $\lambda_{m, n, l}$, equally well always approach infinity quadratically in $m, n$, resp. $l$, as I do not doubt (1). However, such a growth is not sufficient, as is well known, to render the double sum over $m, n$ resp., the triple sum over $m, n, l$, convergent. Rather, here the change of sign of the nominator $u_{m, n}(x) u_{m, n}(\gamma)$ plays an essential role as it is guaranteed in its natural ordering by the oscillatory character of the series." In the above footnote (1) Sommerfeld adds: "The general and rigorous proof of this asymptotic behavior of the eigenvalues seems to me an important and grateful mathematical problem."

Here we have Sommerfeld's conjecture which was one of the motives for the pioneering work of Weyl.

Sommerfeld considers, as an application of his method, the "problem of acoustics of rooms" (using Neumann boundary conditions on the walls), and he emphasizes that his "method is fundamentally different from the classical method introduced in mathematical physics by Fourier", whereby he refers to Fourier's famous work "Théorie [analytique] de la chaleur" from 1822.

Here two remarks are in order. i) In his conjecture, Sommerfeld takes for granted that the eigenvalues depend for example in the three-dimensional case on three integers ("quantum numbers") $(m, n, l)$ i.e. $\lambda_{m, n, l}$, "which each run from 0 to $\infty$ and have the meaning of the number of divisions of the domain by nodal surfaces with respect to the three dimensions. (One may think of the known cases of the parallelepiped or the sphere.)" Consequently, he considers the sum in Equation (1.11) as a triple sum running over $m, n$, and $l$. It is known, however, that the situation envisaged by Sommerfeld holds in general only for domains for which the wave equation (1.1) is separable in coordinates $\left(q_{1}, q_{2}, q_{3}\right)$ i.e. where the particular solutions can be written as a product $u_{m, n, l}=u_{m}\left(q_{1}\right) v_{n}\left(q_{2}\right) w_{l}\left(q_{3}\right)$. In the generic case, however, i.e. for a cavity with arbitrary shape, the eigenvalues depend on a single positive integer only, which just counts the eigenvalues in increasing order, as assumed in (1.11). ii) Sommerfeld points out that the Green function (1.11) "degenerates $(G=\infty)$ " at the points $\lambda=\lambda_{m}$ "according to the general resonance principle, except at special positions of the point source, if, for example, $u_{m}(x)=0$, and therefore the critical eigenvibration is not excited." In the physics literature, the Green function (1.11) is considered as a distribution by adding a small positive imaginary part $(\varepsilon>0)$ to $\lambda \in \mathbb{R}$, i.e. one considers the kernel of the regularized resolvent operator $(\lambda+i \varepsilon+\Delta)^{-1}$. We refer also to Sections 1.3.4, 1.4 and 1.6 where expressions similar to (1.11) are given for the Green's function, for example for the heat kernel. 


\section{2 .3}

\section{The Conjecture of Lorentz (1910)}

At the end of October 1910, i.e. one month after Sommerfeld's talk, Hendrik Antoon Lorentz delivered six lectures at Göttingen under the title "Old and new problems of physics" published [11] from notes taken by Max Born and talked over with Lorentz. See also the letter dated October 28, 1929, sent by Max Born to Einstein, together with Born's comment to this letter from 1969 [12].

Lorentz, who had already received in 1902 the second Nobel prize in physics, was at that time probably the most famous living theoretical physicist. He was invited to Göttingen by the Wolfskehl commission of the Göttingen Academy which was to confer a prize for proving Fermat's last theorem. As long as the prize was not awarded, the proceeds from the principal should be used to invite eminent scientists to lecture at Göttingen. (Paul Wolfskehl (1856-1906), originally a physician, fell ill with multiple sclerosis and then became a mathematician working mainly on number theory; he taught at the Technical University of Darmstadt. The first Wolfskehl lecture was given by Poincaré in 1908 and later lectures were given, among others, by Einstein and Planck; in 1922 Niels Bohr delivered his legendary Wolfskehl lectures on his theory of the atom which later became known as the "Bohr-Festspiele". In 1997 the Wolfskehl prize was given to Andrew Wiles.)

In his last three lectures, Lorentz discussed "the phenomenon of radiating heat". The end of the fourth lecture reads as follows. "In conclusion there is a mathematical problem worth mentioning which perhaps will arouse the interest of mathematicians who are present. It originates in the radiation theory of Jeans. In an enclosure with a perfectly reflecting surface there can form standing electromagnetic waves analogous to tones of an organ pipe; we shall confine our attention only to the very high overtones. Jeans asks for the energy in the frequency interval $\mathrm{d} v$. To this end he first of all calculates the number of overtones which lie between the frequencies $v$ and $v+\mathrm{d} v$ and then multiplies this number by the energy which belongs to the frequency $v$, and which according to a theorem of statistical mechanics is the same for all frequencies. In this manner he gets, indeed, the correct law of the radiation at long wavelengths."

"It is here that there arises the mathematical problem to prove that the number of sufficiently high overtones which lie between $v$ and $v+\mathrm{d} v$ is independent of the shape of the enclosure and is simply proportional to its volume. For several simple shapes, for which the calculation can be carried out, this theorem will be verified in a Leiden dissertation. There is no doubt that it holds in general even for multiply connected spaces. Analogous theorems will also hold for other vibrating structures like elastic membranes and air masses etc."

Weyl, who was present at Lorentz's lectures, writes in a footnote of his second paper [2]: "Lorentz has stated the theorem proven here in Section 1.6 as a plausible conjecture on physical grounds. The simplest cases, for which the proof can be achieved by a direct computation of the eigenvalues, are treated in the Leiden dissertation of Fräulein Reudler." Actually Johanna Reudler verified [13] that the 
asymptotic number of modes depends only on the volume for three special cases, the parallelepiped, the sphere, and the cylinder.

There is an apocryphal report that Hilbert predicted that the theorem would not be proved in his lifetime [9]. Well, as we have seen, he was wrong by many, many years.

Forty years after Lorentz's lectures, Weyl came back to the "eigenvalue problem" [14]: "H.A. Lorentz had impressed upon the mathematicians the urgency for physics of a settlement of this question. For a pupil of Hilbert around 1910 it was natural to visualize the question as one concerning integral equations." In the next section of this paper [14] Weyl draws attention to a more difficult problem by saying: "The physicist will not be satisfied with a knowledge of the asymptotic behavior of the eigenvalues alone; that of the eigenfunctions should also be investigated." And Weyl mentions in this connection Carleman's law, see Section 1.4.3.

Further on in this paper we read the following sentences: "I feel that these informations about the proper oscillations of a membrane, valuable as they are, are still very incomplete. I have certain conjectures on what a complete analysis of their asymptotic behavior should aim at; but since for more than 35 years I have made no serious attempt to prove them, I think I had better keep them to myself."

\subsection{4}

\section{Black Body Radiation: From Kirchhoff to Wien's Law}

The study of the heat radiation from a body in thermal equilibrium with radiation has played an eminent role in the history of physics and mathematics for it led Planck in 1900 to the discovery of the quantum theory and Weyl in 1911 to a first proof of the eigenvalue asymptotics. (There are several historical studies on this subject. Here we rely on the excellent account given by Pais [15] who, however, does not discuss the aspects concerning Weyl's law.) The importance of the heat radiation problem was realized already in 1859 by Gustav Kirchhoff [16]. Let the radiation energy which a body absorbs be converted to thermal energy only, not to any other energy form, and denote by $\mathcal{E}_{v} \mathrm{~d} v$ the amount of energy emitted by the body per unit time per $\mathrm{cm}^{2}$ in the frequency interval $\mathrm{d} v$. (Actually, Kirchhoff uses the wavelength $\lambda$ instead of the frequency $v$.) Furthermore, let $A_{v}$ be its absorption coefficient for frequency $v$. Kirchhoff showed that the ratio $\mathcal{E}_{v} / A_{v}$ is a universal function which depends only on $v$ and the equilibrium (absolute) temperature $T$ and is independent of the shape and any other properties of the body i.e.

$$
\frac{\mathcal{E}_{v}}{A_{v}}=J(v, T) .
$$

A general proof of (1.14) was given much later by Hilbert using the theory of linear integral equations and his "axiomatic method" [17-19].

Kirchhoff called a body perfectly black or just black for short if $A_{v}=1$. Thus $J(v, T)$ is the emitted power of a black body which can be measured if we assume (with Kirchhoff) that a perfect black body can be realized by "a space enclosed by bodies of equal temperature, through which no radiation can penetrate" [16], i.e. by 
an enclosure with perfectly reflecting walls. Kirchhoff challenged theorists and experimentalists alike: "It is a highly important task to find this function $J$. Great difficulties stand in the way of its experimental determination; nevertheless, there appear grounds for the hope that it can be found by experiments because there is no doubt that it has a simple form, as do all functions which do not depend on the properties of individual bodies and which one has become acquainted with before now." [16]

It is worthwhile to mention that Kirchhoff reports in the same paper about his experiments carried out with sunlight, interpreted as heat radiation of very high temperature produced in the atmosphere of the sun, and about his discovery of sodium there. He concludes: "Thus a way is found to ascertain the chemical nature of the atmosphere of the sun, and the same way promises also some information on the chemical nature of the brighter fixed stars." [16]

It will be seen later that Kirchhoff's statement about the shape-independence of $J(v, T)$ implicitly implies part of Weyl's law (1.7) stating that the leading term of the counting function is proportional to the volume $V:=|\Omega|$ of the cavity by which the black body is realized. At this point it is convenient to express $J$ in terms of the spectral energy density $\varrho(v, T)$ which gives the energy per unit volume of the heat radiation in thermal equilibrium at temperature $T$ for frequency $v$ :

$$
J(v, T)=\frac{c}{8 \pi} \varrho(\nu, T)
$$

( $c$ is the velocity of light in vacuo.) It was conjectured by Josef Stefan on experimental grounds in 1879 and proved theoretically by Ludwig Boltzmann in 1884 [20] that the mean total energy $\langle E\rangle(T)$ radiated by the black body is given by the StefanBoltzmann law

$$
\langle E\rangle(T)=V \int_{0}^{\infty} \varrho(v, T) \mathrm{d} v=V \sigma T^{4},
$$

where $\sigma$ is a universal constant (now called the Stefan-Boltzmann constant, whose universal value could only be calculated after the discovery of Planck's law). Boltzmann's proof involves thermodynamics and the electromagnetic theory of Maxwell according to which the mean radiation pressure $\langle p\rangle$ obeys the equation of state

$$
\langle p\rangle=\frac{1}{3} \frac{\langle E\rangle}{V} .
$$

Important progress was made by Wilhelm Wien who proved in 1893 that $\varrho(v, T)$ has to be of the following form (Wien's displacement law) [21]

$$
\varrho(v, T)=v^{3} f(v / T) .
$$

Thus the heat radiation problem was reduced to determining, instead of $J(v, T)$, the universal function $f(x)$ of the single scaling variable $x=v / T$. (Obviously, from (1.17) one immediately derives the Stefan-Boltzmann law (1.16) with $\sigma=\int_{0}^{\infty} x^{3} f(x) \mathrm{d} x$.) Over the years, many proposals for the correct form of $f$ have appeared, see for example the four different forms discussed in [22]. Still, 20 years 
later, Einstein wrote in 1913: "It would be edifying if we could weigh the brain substance which has been sacrificed by the theoretical physicists on the altar of this universal function $f$; and the end of these cruel sacrifices is not yet in sight!" [23]

In 1896 Wien proposed [24] the exponential form $f_{W}(x):=\alpha e^{-\beta x}(\alpha, \beta$ positive constants), that is (Wien's law)

$$
\varrho_{W}(v, T)=\alpha v^{3} e^{-\beta v / T} .
$$

At the same time, Friedrich Paschen carried out precise measurements [22,25] in the near-infrared (for wavelengths $\lambda=c / v=1-8 \mu \mathrm{m}, T=400-1600 \mathrm{~K}$ ) which were in such a good agreement with Wien's law (1.18) that he concluded: "It would seem very difficult to find another function of the two variables $v$ and $T$ [Equation (1.18)] that represents the observations with as few constants." [25]. Thus it appeared that Wien's law was the final answer to the black-body problem.

\subsection{5}

\section{Black Body Radiation: Rayleigh's Law}

In June 1900, that is several months before Planck's revolutionary discovery, Lord Rayleigh made another proposal [26] which for the first time introduces into the black body radiation problem the density of states $D(v)$, that is the density of the vibrational modes of a black body cavity. This step played an important role since Rayleigh's proposal relies on an assumption which 10 years later led to the conjectures of Sommerfeld and Lorentz, and finally to Weyl's law (as already discussed in Sections 1.2.1-1.2.3).

Rayleigh's starting point is the observation that Wien's law (1.18) "viewed from the theoretical side $[. .$.$] appears to me little more than a conjecture ...", and "...$ the law seems rather difficult of acceptance, especially the implication that as the temperature is raised, the radiation of given frequency approaches a limit." [26] Indeed, one obtains from (1.18) $\lim _{T \rightarrow \infty} \varrho_{W}(v, T)=\alpha v^{3}$. He continues: "the question is one to be settled by experiment; but in the meantime I venture to suggest a modification of (1.18), which appears to me more probable a priori." [26]

Without further explanation, Rayleigh assumes, first of all, that the equilibrium distribution $\varrho$ is proportional to the density of the vibrational modes of the cavity per unit volume, that is $\varrho(v, T) \sim D(v) / V$, where

$$
D(v):=\frac{\mathrm{d} \overline{\mathcal{N}}}{\mathrm{d} v} \text { with } \overline{\mathcal{N}}(v):=\bar{N}\left((2 \pi / c v)^{2}\right)
$$

and $\bar{N}(\lambda)$ denotes the leading asymptotic term of the counting function expressed in terms of the frequency $\nu$. Secondly, he assumes according to the "BoltzmannMaxwell doctrine of the partition of energy" (that is the equipartition theorem) that "every mode of vibration should be alike favored ...". Thus he assumes that "the energy should be equally divided among all the modes.... Since the energy in each mode is proportional to $T$ " (that is proportional to $k_{\mathrm{B}} T$ in modern notation, where $k_{\mathrm{B}}$ denotes Boltzmann's constant, introduced actually only later by Planck!), 
Rayleigh's assumption amounts to $\varrho(v, T) \sim(D(v) / V) \cdot T$. As an "illustration" he first considers "the case of a stretched string vibrating transversely" and derives the correct Weyl asymptotics $\bar{N}(\lambda) \sim \sqrt{\lambda}$, that is $\overline{\mathcal{N}}(v) \sim v$ and thus $D(v)=$ constant ("when $v$ is large enough"). Then he continues: "When we pass from one dimension to three dimensions, and consider for example the vibrations of a cubic mass of air, we have ('Theory of Sound', paragraph 267) as the equation for $v^{2}$, $v^{2}=p^{2}+q^{2}+r^{2}$, where $p, q, r$ are integers representing the number of subdivisions in the three directions. If we regard $p, q, r$ as the coordinates of points forming a cubic array, $v$ is the distance of any point from the origin. Accordingly the number of points for which $v$ lies between $v$ and $v+\mathrm{d} v$, proportional to the volume of the corresponding spherical shell, may be represented by $v^{2} \mathrm{~d} v$, and this expresses the distribution of energy according to the Boltzmann-Maxwell law, so far as regards the wavelength or frequency. If we apply this result to radiation, we shall have, since the energy in each mode is proportional to $T, T v^{2} \mathrm{~d} v \ldots$.. [26] Thus Rayleigh obtains (apart from the numerical coefficient) the correct Weyl asymptotics for a three-dimensional cavity, that is $\bar{N}(\lambda) \sim V \lambda^{3 / 2}$, see (1.5), which leads to $\overline{\mathcal{N}}(v) \sim V v^{3}$ or $D(v) / V \sim v^{2}$, and thus to

$$
\varrho_{\mathrm{REJ}}(v, T)=c_{1} v^{2} T
$$

which is commonly known as the Rayleigh-Jeans law but which should rather be referred to as the Rayleigh-Einstein-Jeans law. (We shall discuss below Einstein's, Rayleigh's second, and Jeans' derivation of (1.19) which includes also the explicit expression for the coefficient $c_{1}$.)

Here several remarks are in order. i) It is obvious that Rayleigh did not worry about the fact that he used the scalar wave equation in his derivation of the mode asymptotics, by referring to his famous book on acoustics [27], instead of the vector Maxwell equations, which were studied only later by Weyl $[2,4,5]$. ii) In deriving the vibrational mode asymptotics for a cubical box, Rayleigh takes for granted that the result $\overline{\mathcal{N}}(v) \sim V v^{3}$ holds for any shape of the cavity and thus concludes that $D(v) / V$ is independent of the shape. In other words, Rayleigh assumes to be true what 10 years later was formulated as a conjecture by Sommerfeld and Lorentz. iii) Although his derivation of $D(v) / V \sim v^{2}$ holds only asymptotically for $v \rightarrow \infty$, he derives from this result the law (1.19) stating that it may have the proper form when $v / T$ is small! iv) Rayleigh observes that (1.19) is of the general scaling form (1.17) (with $f_{\mathrm{REJ}}(x)=c_{1} / x$ ), and he regards this "as some confirmation of the suitability of (1.19)." v) Without further comment, Rayleigh writes in his paper [26]: "If we introduce the exponential factor, the complete expression will be

$$
\varrho_{\mathrm{R}}=c_{1} v^{2} \mathrm{Te}^{-c_{2} v / T}
$$

It is this expression which became known as the Rayleigh law. vi) There is no doubt that Rayleigh must have realized that (1.19) is entirely unacceptable since the quadratic dependence of $v$ leads to a physically meaningless divergence (later called "ultraviolet catastrophe" by Ehrenfest) of the total radiation energy (see (1.16)). Of course, by multiplying with the exponential "convergence factor" taken over from 
Wien's law (1.18), the divergence is avoided and the Stefan-Boltzmann law (1.16) holds with $\sigma=2 c_{1} / c_{2}^{3}$.

At the end of his paper Rayleigh writes [26]: "Whether (1.20) represents the facts of observation as well as (1.18) I am not in a position to say. It is to be hoped that the question may soon receive an answer at the hands of the distinguished experimenters who have been occupied with this subject."

We can assume that Rayleigh was well informed about the two teams working in Berlin on black body radiation experiments. The first of these, Otto Lummer and Ernst Pringsheim, had already shown in February 1900 that Wien's law (1.18) fails in the wavelength region $\lambda=12-18 \mu \mathrm{m}$ (for $T=300-1650 \mathrm{~K}$ ) [28]. The second team, Heinrich Rubens and Ferdinand Kurlbaum, presented their measurements in the even further infrared $\left(\lambda=30-60 \mu \mathrm{m}, T=-188-1500^{\circ} \mathrm{C}\right)$ to the Prussian Academy on October 25, 1900 [29]. In a famous figure, they plotted $\varrho$ as a function of $T$ at the fixed wavelength $\lambda=51.2 \mu \mathrm{m}$ and compared their data with some theoretical curves. One of these was the Wien curve, another the Rayleigh curve. Both curves did not work! But then we read in the paper [29] that they had compared their data with a "fifth formula, given by Herr M. Planck after our experiments had already been concluded ..." and which "reproduces our observation within the limits of error."

\subsection{6}

\section{Black Body Radiation: Planck's Law and the Classical Limit}

According to Pais [15], Planck probably discovered his law in the early evening of Sunday, October 7, 1900, Rubens and his wife had called on the Plancks on the afternoon of that day. In the course of conversation, Rubens mentioned to Planck that he had found $\varrho(v, T)$ to be proportional to $T$ for small $v$. Planck went to work after the visitors left and found an interpolation between his results and Wien's law, Equation (1.18). He communicated his formula by postcard to Rubens the same evening and stated it publicly [30] in a discussion remark on October 19, following the presentation of a paper by Kurlbaum. Expressed in notations introduced by Planck two months later, Planck's law reads:

$$
\varrho_{P}(v, T)=\frac{8 \pi h v^{3}}{c^{3}} \frac{1}{e^{h v / k_{\mathrm{B}} T}-1},
$$

where $h$ denotes Planck's constant and $k_{\mathrm{B}}$ is Boltzmann's constant.

Let us consider two limits of Planck's law. First, in the high-frequency or lowtemperature regime, which is now identified as the quantum limit in which the photon energy $h v$ is much larger than the thermal energy $k_{\mathrm{B}} T$, that is $h v / k_{\mathrm{B}} T \gg$ 1 , we recover Wien's law (1.18) with $\alpha=(8 \pi h) / c^{3}$ and $\beta=h / k_{\mathrm{B}}$. This explains why Paschen's experiments [22, 25], for which $h v / k_{\mathrm{B}} T \approx 15$ holds, were in such a good agreement with Wien's law, as already mentioned. At the other extreme of low frequency or high temperature, $h v / k_{\mathrm{B}} T \ll 1$, which is obtained from Planck's law in the formal limit when Planck's constant approaches zero, $h \rightarrow 0$, and is now identified as the semiclassical limit, we recover the Rayleigh-Einstein-Jeans 
law (1.19)

$$
\varrho_{\mathrm{P}}(v, T)=\frac{8 \pi v^{2}}{c^{3}}\left(k_{\mathrm{B}} T\right)[1+O(h)] \quad(h \rightarrow 0) .
$$

A comparison with (1.19) gives the correct value for the constant $c_{1}$ left undetermined by Rayleigh, that is

$$
c_{1}=\frac{8 \pi k_{\mathrm{B}}}{c^{3}}=\frac{8 \pi}{c^{3}} \frac{R}{N_{\mathrm{A}}},
$$

which does not depend on $h$, and where $R$ is the gas constant and $N_{\mathrm{A}}$ is Avogadro's number.

Since our main interest here is to understand the role played by Weyl's law, we are not discussing at this point the arguments using "resonators" which led Planck to his formula. (Planck's original derivation does not refer to the vibrations of the cavity and thus does not involve the density of states.) Using the fact that the correct formula for the radiating heat, that is Planck's formula, in the classical limit exactly takes the form of the Rayleigh-Einstein-Jeans law, we can interpret the latter in purely classical terms by making the general ansatz (valid only for $h=0$ !)

$$
\varrho_{\text {class }}(v, T):=\lim _{V \rightarrow \infty}\left(\frac{D_{\mathrm{em}}(v)}{V}\right) k_{\mathrm{B}} T,
$$

where $D_{\mathrm{em}}(v)$ denotes the density of states of the electromagnetic vibrations in a cavity of volume $V$. Furthermore, we have taken care of the fact that the predictions of thermodynamics involve the so-called thermodynamic limit $V \rightarrow \infty$. Here

$$
D_{\mathrm{em}}(v):=\frac{\mathrm{d} \overline{\mathcal{N}}_{\mathrm{em}}(v)}{\mathrm{d} v} \text { with } \overline{\mathcal{N}}_{\mathrm{em}}(v)=2 \overline{\mathcal{N}}(v)=2 \bar{N}\left((2 \pi / c v)^{2}\right)
$$

where $\bar{N}(\lambda)$ denotes the two asymptotic terms of the counting function (1.7) for the three-dimensional case, and the factor 2 comes from the two polarizations of the photon. We then obtain

$$
\overline{\mathcal{N}}_{\mathrm{em}}(v)=V \frac{8 \pi}{3 c^{3}} v^{3}+O_{|\partial \Omega|}\left(v^{2}\right)
$$

which leads to

$$
\lim _{V \rightarrow \infty}\left(\frac{D_{\mathrm{em}}(v)}{V}\right)=\frac{8 \pi}{c^{3}} v^{2}
$$

since $\lim _{V \rightarrow \infty}(|\partial \Omega| / V)=0$, where $|\partial \Omega|$ denotes the surface area of the cavity.

In his famous book, originally published in 1928 in German under the title "Gruppentheorie und Quantenmechanik" [31, p. 103-104 and p. 402] Weyl treats the black-body radiation and proves that it "is mathematically equivalent to a system of infinitely many oscillators." He then states, without proof: "For high frequencies $v$ there are approximately $V\left(8 \pi v^{2} \mathrm{~d} v / c^{3}\right)$ modes of oscillation in the frequency interval $v, v+\mathrm{d} v$. We are interested above all in the limiting case of an 
infinitely large cavity; the spectrum then becomes continuous and our formula for the density of frequencies becomes exact." In a footnote he adds: "This result is easily obtained by elementary methods for a rectangular parallelepiped. For the general proof see $\mathrm{H}$. Weyl $[4,5,8]$." It is clear that the limit $V \rightarrow \infty$ is an idealization which can never be realized in a physical experiment. Rather the "assumption must always hold that the linear dimensions of all cavities considered and also the curvature of the radii of all surfaces considered must be large compared with the wavelengths of the radiation. Then we are allowed, without making a noticeable error, to neglect the influences of the form of the boundaries caused by diffraction." [32, p. 2]

Inserting (1.26) into (1.24), one obtains

$$
\varrho_{\text {class }}(v, T)=\frac{8 \pi k_{\mathrm{B}}}{c^{3}} v^{2} T
$$

which is precisely the Rayleigh-Einstein-Jeans law (1.19) with the correct power behavior in $v$ and the same coefficient (1.23) as obtained from the exact Planck formula. It is thus seen that heat radiation (in the classical limit) is indeed independent of the shape of the cavity due to Weyl's law and the performance of the thermodynamical limit.

As shown above, Planck's radiation law (1.21) from October 1900 can be considered as a simple interpolation formula which smoothly interpolates between the Rayleigh-Einstein-Jeans law (1.27) and Wien's law (1.18). In fact, it differs from Wien's law only by the -1 in the denominator. It has rightly been said [15], that even if Planck had stopped after October 19, he would forever be remembered as the discoverer of the radiation law. It is a true measure of his greatness that he went further. He wanted to interpret (1.21). That made him to discover the quantum theory. Already on December 14, 1900, Planck presented a theoretical derivation of his formula to the German Physical Society in Berlin [33] and shortly afterwards (7 January 1901) submitted his famous paper [34]. More and more precise measurements carried out during the following years established Planck's formula as the correct phenomenological law of black body radiation. It is thus quite astonishing to learn that several excellent theoretical physicists, in particular Lorentz, Lord Rayleigh, and Jeans, worked on alternative theories leading to formulae different from Planck's. Ironically, since Planck's derivation does not rely on the density of states, the origin of Weyl's law lies just in these alternative approaches. Therefore, a history of Weyl's law without a discussion of these differing theories would be incomplete.

\subsection{7}

\section{Black Body Radiation: The Rayleigh-Einstein-Jeans Law}

First of all, one should understand why some theorists were seeking for different theories of black body radiation despite the great empirical success of Planck's formula. The explanation is quite obvious: they realized that Planck's radiation theory was not satisfactory from a theoretical point of view; in fact, it was inconsistent! As 
the above quotation from Einstein [23] shows, the problem still existed in 1913; the ultimate derivation of Planck's formula was only provided in 1924 using the correct Bose-Einstein quantum statistics.

In 1903, Lorentz [35] derived (1.19) in the low-frequency limit together with the value $c_{1}=\left(16 \pi \alpha / 3 c^{3}\right)$ for the coefficient $c_{1}$ where $\alpha$ is a constant such that $\alpha T$ represents the mean kinetic energy of a molecule of a gas. Comparing (1.19) with the low-frequency limit of Planck's formula (1.21), he obtained $\alpha=(3 / 2) k_{\mathrm{B}}$ (see also (1.23)) and states: "Now the mean kinetic energy of a molecule of a gas would be $(3 / 2) k T$ according to Planck ... there appears therefore to be full agreement between the two theories in the case of long waves, certainly a remarkable conclusion, as the fundamental assumptions are widely different."

The year 1905 is one of the most amazing ones in the history of science: it marks, first of all, Einstein's annus mirabilis with his five seminal papers, where only the first one on the famous light quantum hypothesis [36] concerns us here, since it deals with the radiation problem, and, secondly, the series of papers published by Rayleigh [37, 38] and Jeans [39-42] on the radiation problem using the Weyl asymptotics.

From reading these papers it becomes clear that Einstein is the only one who takes Planck's formula serious since it "agrees with all experiments to date" [36]. But in Section 1.1 of this paper entitled "On a difficulty concerning the theory of the «black radiation »" [36] he implicitly expresses his doubts on Planck's derivation by showing that Planck should have obtained (1.27) instead of his formula (1.21)! The argument is very simple. Planck's starting point in his derivation is the formula

$$
\varrho(v, T)=\frac{8 \pi v^{2}}{c^{3}}\langle E\rangle(v, T),
$$

where $\langle E\rangle(v, T)$ is the average energy of a Planck resonator of frequency $v$ at the joint equilibrium of matter and radiation at temperature $T$. Furthermore, the equilibrium energy of a one-dimensional resonator is according to the equipartition theorem given by $\langle E\rangle(v, T)=k_{\mathrm{B}} T$, and inserting this into (1.28), Einstein obtains (1.27). We thus see that the radiation law (1.27), commonly known as the Rayleigh-Jeans law, ought to be called the Rayleigh-Einstein-Jeans law [15]. Many years later Einstein said: "If Planck had drawn this conclusion, he probably would not have made his great discovery, because the foundation would have been withdrawn from his deductive reasoning." [43]

Years later Planck himself presented two derivations of (1.27) in his famous book “Theorie der Wärmestrahlung" [32] and concluded: "It is not too much asserted if we say in generalizing: The classical theory leads of necessity to Rayleigh's radiation law."

Einstein's paper [36] was submitted on 17 March 1905, and thus is the earliest among the above mentioned papers by Rayleigh and Jeans. (Rayleigh's first paper [37] was submitted on 6 May 1905; Jeans' first paper on radiation [39] on 20 May 1905.)

As discussed in Section 1.2.5, Rayleigh was the first [26] to have already counted in 1900 "the number of modes corresponding to any finite space occupied by 
radiation" [37] and to obtain the law (1.19), however, without determining the coefficient $c_{1}$. "Elicited by the very clear statement of his view which Mr. Jeans gives in NATURE of April 27 (1900) [44]", he repeats the arguments of his former paper [26] "with an extension designed to determine the coefficient as well as the law of radiation" [37]. By counting the modes within a cube of length $l$ (Weyl's law), he obtains again (1.19) "as probably representing the truth when $v$ is small." He remarks that this formula agrees with Planck's in the limit when $v$ is small apart from the fact that his value for $c_{1}$ "is eight times as large as that found by Planck." Rayleigh adds: "A critical comparison of the two processes would be of interest, but not having succeeded in following Planck's reasoning I am unable to undertake it. As applying to all wavelengths, his formula would have the greater value if satisfactorily established. On the other hand, the reasoning leading to (1.19) is very simple, and this formula appears to me a necessary consequence of the law of equipartition as laid down by Boltzmann and Maxwell. My difficulty is to understand how another process, also based on Boltzmann's ideas, can lead to a different result." [37]

Two days after Rayleigh's letter [37] Jeans submitted a short letter [39] in reply to Rayleigh. His main point was "the general question of the applicability of the theorem of equipartition to the energy of the ether" as opened up by Rayleigh. He takes up "Lord Rayleigh's example of a stretched string, say a piano wire" and then discusses the "vibrations of the ether in a finite enclosure". He writes: "It is easily seen that the number of slow vibrations is approximately proportional to the volume of the enclosure, so that roughly the energy of ether must be measured per unit volume in order to be independent of the size of the enclosure." He then arrives at (1.19), but without determining the value for $c_{1}$. On June 7, Jeans adds a "postscript" to his paper [40] and calculates again "the number of degrees of freedom of the æther" by referring to Rayleigh's book [27](!). From this he obtains the radiation law (1.19) together with the correct value (1.23) for the coefficient $c_{1}$. "This is one-eighth of the amount found by Lord Rayleigh, but agrees exactly with that given by Planck for large values of $\lambda$. It seems to me that Lord Rayleigh has introduced an unnecessary factor 8 by counting negative as well as positive values of his integers $p, q, r . "$ (See the discussion before equation (1.19).) A month later, Rayleigh replies to Jeans [38]: "In NATURE, May 18, I gave a calculation of the coefficient of complete radiation at a given absolute temperature for waves of great length on principles laid down in 1900, and it appeared that the result was eight times as great as that deduced from Planck's formula for this case. In connection with similar work of his own, Mr. Jeans has just pointed out that I have introduced a redundant factor 8 by counting negative as well as positive values of my integers $p, q$, $r$ - I hasten to admit the justice of this correction. But while the precise agreement of results in the case of very long waves is satisfactory so far as it goes, it does not satisfy the wish expressed in my former letter for a comparison of processes. In the application to waves that are not long, there must be some limitation on the principle of equipartition. Is there any affinity in this respect between the ideas of Prof. Planck and those of Mr. Jeans?"

On July 27, Jeans published another letter [41]: "On two occasions (NATURE, May 18 and July 13) Lord Rayleigh has asked for a critical comparison of two the- 
ories of radiation, the one developed by Prof. Planck and the other by myself, following the dynamical principles laid down by Maxwell and Lord Rayleigh. It is with the greatest hesitation that I venture to express my disagreement with some points in the work of so distinguished a physicist as Prof. Planck, but Lord Rayleigh's second demand for a comparison of the two methods leads me to offer the following remarks, which would not otherwise have been published, on the theory of Prof. Planck." Jeans then criticises Planck's concept of the "entropy of a single resonator" given by the formula $S=k_{\mathrm{B}} \log W+$ constant by saying: "The function $W$, as at present defined, seems to me to have no meaning. Planck (in common, I know, with many other physicists) speaks of the 'probability' of an event, without specifying the basis according to which the probability is measured. This conception of probability seems to me an inexact conception, and as such to have no place in mathematical analysis." [41]

Jeans' critique of Planck's derivation is fully justified as one can infer from Einstein's "laudatio" for Planck written in 1913: “This [that is Planck's] calculation which, due to the not sufficiently sharp definition of $W$, could not be performed without arbitrariness, led to the radiation formula (1.21) ..." [23].

Jeans then continues [41] by criticising Planck's introduction of his famous constant $h$ via the fundamental relation $\varepsilon=h v$. "Here $\varepsilon$ is a small quantity, a sort of indivisible atom of energy, introduced to simplify the calculations. We may legitimately remove this artificial quantity by passing to the limit in which $\varepsilon=0 \ldots$ The relation $\varepsilon=h v$ is assumed by Planck in order that the law ultimately obtained may satisfy Wien's 'displacement law' i.e. may be of the form (1.17). This law is obtained by Wien from thermodynamical considerations on the supposition that the energy of the ether is in statistical equilibrium with that of matter at a uniform temperature. The method of statistical mechanics, however, enables us to go further and determine the form of the function $f(v / T)$; it is found to be $8 \pi k_{\mathrm{B}}(T / v)$, so that Wien's law (1.17) reduces to the law given by expression (1.27). In other words, Wien's law directs us to take $\varepsilon=h v$, but leaves $h$ indeterminate, whereas statistical mechanics gives us the further information that the true value of $h$ is $h=0$. Indeed, this is sufficiently obvious from general principles. The only way of eliminating the arbitrary quantity $\varepsilon$ is by taking $\varepsilon=0$, and this is the same as $h=0$. - Thus it comes about that in Planck's final law (1.21) the value of $h$ is left indeterminate; on putting $h=0$, the value assigned to it by statistical mechanics, we arrive at once at the law (1.27).... I carry the method further than Planck, since Planck stops short of the step of putting $h=0$. I venture to express the opinion that it is not legitimate to stop short at this point, as the hypotheses upon which Planck has worked lead to the relation $h=0$ as a necessary consequence. Of course, I am aware that Planck's law is in good agreement with experiment if $h$ is given a value different from zero, while my own law, obtained by putting $h=0$, cannot possibly agree with experiment. This does not alter my belief that the value $h=0$ is the only value which it is possible to take." [41]

Although Jeans' conclusion [41] that Planck should have arrived at the radiation law (1.27) instead of his formula (1.21) agrees with the conclusions drawn earlier by Einstein [36] and Rayleigh [37, 38]; his belief that the value $h=0$ is the only 
value which Planck's constant can possibly take shows that he did not realize the importance of the equation $\varepsilon=h v$ (neither did Planck nor Rayleigh!). It was Einstein's revolutionary light-quantum paper [36] (the only contribution he himself called revolutionary) which gave a deep meaning to this equation and thus paved the way towards a quantum theory. Einstein put forward the following "heuristic view" [36]. "Monochromatic radiation of low density (within the domain of validity of Wien's radiation formula) behaves in thermodynamic respect as if it would consist of mutually independent energy quanta of magnitude $R \beta v / N_{\mathrm{A}}$ [ $\equiv h v$ using $\left.\beta=h / k_{\mathrm{B}}\right]$. - If, in regard to the volume dependence of the entropy, monochromatic radiation (of sufficiently low density) behaves as a discontinuous medium, which consists of energy quanta of magnitude [ $h v]$, then this suggests an inquiry as to whether the laws of the generation and conservation of light are also constituted as if light were to consist of energy quanta of this kind."

\subsection{8}

\section{From Acoustics to Weyl's Law and Kac's Question}

In the previous sections we have discussed how the heat radiation problem was at the origin of Weyl's famous work. Furthermore, we have seen that the idea of expressing the spectral energy density $\varrho(v, T)$ of the black body radiation in terms of the density of states $D(v)$ goes back to Rayleigh [26] who in turn reduced the problem to the "vibrations of a cubical mass of air". Thus Weyl's law actually has its roots in acoustics. In view of the fact that Rayleigh was a leading expert in acoustics and the author of the famous book "The Theory of Sound" [27], first published in 1877, it is not surprising that he realized that the radiation problem can be related to the number of vibrational modes of a black body cavity. All the more reason that it is strange to observe that he had difficulties in obtaining the correct value for the constant $c_{1}$ in his radiation law (1.19). The problem was of course a question of the correct boundary conditions in the electromagnetic case. In his book, Rayleigh writes: "Some of the natural notes of the air contained within a room may generally be detected on singing the scale. Probably it is somewhat in this way that blind people are able to estimate the size of rooms." [27] And in a footnote he adds: "A remarkable instance is quoted in Young's Natural Philosophy, II. p. 272, from Darwin's Zoonomia, II. 487. “The late blind Justice Fielding walked for the first time into my room, when he once visited me, and after speaking a few words said, 'This room is about 22 feet long, 18 wide, and 12 high'; all which he guessed by the ear with great accuracy." And then Rayleigh continues: "In long and narrow passages the vibrations parallel to the length are too slow to affect the ear, but notes due to transverse vibrations may often be heard. The relative proportions of the various overtones depend upon the place at which the disturbance is created. In some cases of this kind the pitch of the vibrations, whose direction is principally transverse, is influenced by the occurrence of longitudinal motion...."

These remarks on acoustics lead us directly to Kac's famous question: "Can one hear the shape of a drum?" [9], which will be discussed in Sections 1.3.6 and 1.3.7, 
and the more general question: "Can one hear the periodic orbits of a drum?" to be discussed in Section 1.3.7.

\section{3}

\section{Weyl's Law with Remainder Term. I}

\subsection{1}

The Laplacian on the Flat Torus $\mathbb{T}^{2}$

In special cases it is possible to derive exact formulae for the counting function $N(\lambda)$ which contain in addition to the Weyl term (and possible higher order terms) an explicit expression for a remainder function. The most elegant way to derive these formulae is based on trace formulae; a famous example is the Selberg trace formula [45-47] to be discussed in Section 1.4. To illustrate the method in a simple case, we consider the eigenvalue problem $-\Delta_{\mathbb{T}^{2}} u=\lambda u$, where $\Delta_{\mathbb{T}^{2}}$ denotes the Laplacian on a flat torus $\mathbb{T}^{2}:=S_{L}^{1} \times S_{L}^{1}=\mathbb{R}^{2} /(L \mathbb{Z} \times L \mathbb{Z})$ characterized by a length scale $L>0 . \mathbb{T}^{2}$ can be represented by the fundamental domain $\Omega=[0, L] \times[0, L] \in \mathbb{R}^{2}$ i.e. by a square with side $L$, where opposite sides are glued together. Obviously, all of $\mathbb{R}^{2}$ is covered by the $\Gamma$-translates of $\Omega$ where $\Gamma$ is the translation group $(L \mathbb{Z})^{2}$. This produces a tessellation of $\mathbb{R}^{2}$ and leads to the periodic boundary conditions

$$
u\left(x_{1}+\mu_{1} L, x_{2}+\mu_{2} L\right)=u\left(x_{1}, x_{2}\right), \quad\left(x_{1}, x_{2}\right) \in \Omega,\left(\mu_{1}, \mu_{2}\right) \in \mathbb{Z}^{2} .
$$

Note that $\mathbb{T}^{2}$ is a smooth, compact manifold with area $|\Omega|=L^{2}$ (but with no boundary). It is easy to see that $\left(e_{m}\right)_{m \in \mathbb{Z}^{2}}=\left(e^{2 \pi i(m \cdot x) / L}\right)_{m \in \mathbb{Z}^{2}}$ is an orthonormal basis of $L^{2}(\Omega)$ consisting of eigenvectors of $-\Delta_{\mathbb{T}^{2}}$ with discrete eigenvalues $\left(\lambda_{m}\right)_{m \in \mathbb{Z}^{2}}=$ $\left(\left(4 \pi^{2}\right) / L^{2}\left(m_{1}^{2}+m_{2}^{2}\right)\right)_{\left(m_{1}, m_{2}\right) \in \mathbb{Z}^{2}}$.

Let $r(n)=\#\left\{\left(m_{1}, m_{2}\right) \in \mathbb{Z}^{2}, n=m_{1}^{2}+m_{2}^{2}\right\}, n \in \mathbb{N}_{0}$, with $r(0)=1$, i.e. $r(n)$ denotes the number of representations of $n \in \mathbb{N}_{0}$ as a sum of two squares of integers. Obviously, the distinct eigenvalues of $-\Delta_{\mathbb{T}^{2}}$,

$$
\left(\bar{\lambda}_{n}\right)_{n \in \mathbb{N}_{0}}=\left(\frac{4 \pi^{2}}{|\Omega|} n\right)_{n \in \mathbb{N}_{0}},
$$

occur with multiplicity $r(n)$. Then the counting function on the torus reads

$$
N(\lambda)=\sum_{\bar{\lambda}_{n} \leq \lambda} r(n)=\sum_{0 \leq n \leq\left(|\Omega| / 4 \pi^{2}\right) \lambda} r(n) .
$$

The very irregular ("valde irregulariter" [48]) number theoretical function $r(n)$ had already been studied by Gauss [48] who derived the formula $r(n)=4\left(d_{1}(n)-d_{3}(n)\right)$, $n \geq 1$, where $d_{1}(n)$ and $d_{3}(n)$ are the number of divisors of $n$ of the form $4 m+1$ and $4 m+3, m \in \mathbb{N}_{0}$, respectively. The first values are $r(0)=1, r(1)=4, r(2)=4, r(3)=0$, $r(4)=4, r(5)=8$. If $n \equiv 3(\bmod 4)$ then $r(n)=0$. For large $n$ one has $r(n)=O\left(n^{\varepsilon}\right)$ for every $\varepsilon>0 ; r(n)=O\left((\log n)^{\delta}\right)$ is false for every $\delta$. The average order of $r(n)$ is

$$
\bar{r}:=\lim _{x \rightarrow \infty} \frac{1}{x} \sum_{0 \leq n \leq x} r(n)=\pi
$$


(Gauss resp. the Weyl law, see (1.31)). For further information about $r(n)$, see [49, pp. 241].

\subsection{2}

The Classical Circle Problem of Gauss

Let

$$
v(x):=\sum_{0 \leq n \leq x} r(n)=\sum_{\substack{m_{1}^{2}+m_{2}^{2} \leq x \\\left(m_{1}, m_{2}\right) \in \mathbb{Z} \times \mathbb{Z}}} 1,
$$

then

$$
N(\lambda)=v\left(\frac{|\Omega|}{4 \pi^{2}} \lambda\right)
$$

and the derivation of Weyl's law is reduced to a lattice point problem, since $v(x)$ has a simple geometric interpretation as the number of lattice points in the interior and on the boundary of a circle with center $(0,0)$ and of radius $\sqrt{x}$. The problem of calculating the leading asymptotic behavior of $v(x)$ for $x \rightarrow \infty$ was already considered by Gauss in 1834 [48] (see also [50, pp. 32-39]). He realized that $v(x)$ is approximately given by the sum of the areas of all squares of unit side length which are inscribed in the circle of radius $\sqrt{x}$, and thus $v(x)$ is in first approximation equal to the area of the circle $\pi(\sqrt{x})^{2}=\pi x$. Actually, Gauss proved

$$
\lim _{x \rightarrow \infty} \frac{v(x)}{x}=\pi
$$

which implies Weyl's law

$$
\lim _{\lambda \rightarrow \infty} \frac{N(\lambda)}{\lambda}=\frac{|\Omega|}{4 \pi}
$$

for the counting function (1.29). Based on his result (1.31), Gauss considered $v(x) / x$ as an approximation method to calculate $\pi$. To this purpose, he thought about the error one makes at finite $x$. Again, by geometrical intuition, one sees that the error should not be larger than the combined area of those squares that are cut by the boundary of the circle i.e. those contained in an annulus of width $2 \sqrt{2}$, and thus is approximately given by $2 \sqrt{2}$ times the perimeter of the circle $2 \pi \sqrt{x}$, and, indeed, Gauss was able to prove

$$
v(x)=\pi x+O(\sqrt{x}) \quad(x \rightarrow \infty)
$$

which implies

$$
N(\lambda)=\frac{|\Omega|}{4 \pi} \lambda+O(\sqrt{\lambda}) \quad(\lambda \rightarrow \infty) .
$$

Defining a remainder term $P(x)$,

$$
v(x)=\pi x+P(x)
$$


we are led to the classical circle problem, a famous problem in analytic number theory [51, pp. 181-308]: estimate the remainder function $P(x)$ as accurately as possible. In particular, determine $\alpha_{0}=\inf \alpha$ in the estimate

$$
P(x)=O\left(x^{\alpha}\right) \quad(x \rightarrow \infty) .
$$

In Figures 1.1 and 1.2 we show plots of $v(x)$ and $P(x)$, respectively, from which it becomes clear that $P(x)$ - due to the erratic behavior of $r(n)$ - is a very irregular function wildly fluctuating about zero. It is therefore no big surprise that to determine the actual size of $P(x)$, and thus the remainder to Weyl's law, is a difficult problem. Considering the difference $v(n+1 / 2)-v(n), n \in \mathbb{N}$, it is easy to see that $P(x)=o(1)$ is false, and thus $0 \leq \alpha_{0} \leq 1 / 2$. An important result showing that $P(x)$ is much smaller than the classical result $\alpha_{0} \leq 1 / 2$ is due to Sierpiński who proved $\alpha_{0} \leq 1 / 3$ in 1906 [52, pp. 73-108]. A famous conjecture by Hardy from 1915 states that $\alpha_{0}$ should be $1 / 4$, i.e. $P(x)=O\left(x^{1 / 4+\varepsilon}\right)$ for every $\varepsilon>0[53,54]$. Actually, Hardy proved $\alpha_{0} \geq 1 / 4$.

During the last 100 years, the values for $\alpha_{0}$ decreased only by a tiny amount: $\alpha_{0} \leq 37 / 112=0.330 \ldots$ (van der Corput 1923 [55]), $\alpha_{0} \leq 12 / 37=0.324 \ldots$ (Wen-Lin Yin 1962 [56]), $\alpha_{0} \leq 7 / 22=0.318 \ldots$ (Iwaniec and Mozzochi 1988 [57]). The best bound known today is due to Huxley who proved in 1992 that

$$
P(x)=O\left(x^{23 / 73}(\log x)^{315 / 146}\right) ;
$$

note that $23 / 73=0.315 \ldots$ is still far away from $1 / 4$ ! (For a review, see [58].) Since $P(x)$ is a wildly fluctuating function, it might be that some very rare spikes exceeding the conjectured $x^{1 / 4}$-behavior make it extremely difficult to improve the best existing bound. In order to "tame" these spikes, one can consider moments of $P(x)$ and hope that the spikes are being washed out. We shall come back to this idea in Section 1.3.9 making use of the trace formula for $v(x)$ which we shall now derive.

Note added in proof: in a recent unpublished paper [59] it is claimed to present a proof of Hardy's conjecture.

\subsection{3}

\section{The Formula of Hardy-Landau-Voronoï}

The counting function $v(x)$ can be rewritten as

$$
v(x)=\sum_{m \in \mathbb{Z}^{2}} \theta\left(x-m^{2}\right)
$$

where $\theta(x)$ denotes the Heaviside step function. Instead of $\theta\left(x-m^{2}\right)$, let us consider a function $g(m)$ with

- $g: \mathbb{R}^{2} \rightarrow \mathbb{C}$, continuous

- $g(x)=O\left(1 /\left(\|x\|^{2+\varepsilon}\right)\right)$ for $\|x\|^{2}=x_{1}^{2}+x_{2}^{2} \rightarrow \infty, \varepsilon>0$,

and let us study the sum $\sum_{m \in \mathbb{Z}^{2}} g(m)$. Using the Poisson summation formula, we obtain

$$
\sum_{m \in \mathbb{Z}^{2}} g(m)=\sum_{l \in \mathbb{Z}^{2}} \tilde{g}(l)
$$


where $\tilde{g}$ denotes the Fourier transform of g:

$$
\tilde{g}(l)=\int_{\mathbb{R}^{2}} g(x) e^{-2 \pi i(l \cdot x)} \mathrm{d}^{2} x .
$$

To apply this to the circle problem, we make the further assumption that $g(x)$ is a radial function which depends only on $\varrho=\|x\|$, i.e.

$$
g(x)=g\left(x_{1}, x_{2}\right)=\phi\left(x_{1}^{2}+x_{2}^{2}\right)=\phi\left(\varrho^{2}\right) .
$$

Thus

$$
\begin{aligned}
\tilde{g}(l) & =\tilde{g}\left(l_{1}, l_{2}\right)=\int_{-\infty}^{\infty} \int_{-\infty}^{\infty} \phi\left(x_{1}^{2}+x_{2}^{2}\right) e^{-2 \pi i\left(l_{1} x_{1}+l_{2} x_{2}\right)} \mathrm{d} x_{1} \mathrm{~d} x_{2} \\
& =\int_{0}^{\infty} \varrho \phi\left(\varrho^{2}\right) \int_{0}^{2 \pi} e^{-2 \pi i\|l\| \varrho \cos \varphi} \mathrm{d} \varphi \mathrm{d} \varrho=2 \pi \int_{0}^{\infty} \varrho \phi\left(\varrho^{2}\right) J_{0}(2 \pi\|l\| \varrho) \mathrm{d} \varrho .
\end{aligned}
$$

Here we have introduced polar coordinates in $\mathbb{R}^{2}, x_{1}=\varrho \cos \varphi, x_{2}=\varrho \sin \varphi, 0 \leq \varphi \leq$ $2 \pi$, and have used the integral representation

$$
J_{0}(z)=\frac{1}{2 \pi} \int_{0}^{2 \pi} e^{-i z \cos \varphi} \mathrm{d} \varphi
$$

for the Bessel function $J_{0}(z)$. Now the Poisson summation formula (1.33) reads

$$
\sum_{m \in \mathbb{Z}^{2}} \phi\left(m^{2}\right)=2 \pi \sum_{l \in \mathbb{Z}^{2}} \int_{0}^{\infty} \varrho \phi\left(\varrho^{2}\right) J_{0}(2 \pi\|l\| \varrho) \mathrm{d} \varrho,
$$

or, by introducing the multiplicity $r(n)$ and $\varrho=\sqrt{x}, x \geq 0$ :

$$
\sum_{n=0}^{\infty} r(n) \phi(n)=\pi \sum_{n=0}^{\infty} r(n) \int_{0}^{\infty} \phi(x) J_{0}(2 \pi \sqrt{n x}) \mathrm{d} x .
$$

This is the theorem due to Hardy [54, 60, 61], Landau [51, pp. 189] and Voronoi [62].

1.3.4

The Trace Formula on the Torus $\mathbb{T}^{2}$ and the Leading Weyl Term

We recall that the distinct eigenvalues on the torus $\mathbb{T}^{2}$ are given by $\bar{\lambda}_{n}=(2 \pi / L)^{2} n=$ $p_{n}^{2}$ with $p_{n}:=(2 \pi / L) \sqrt{n}, n \in \mathbb{N}_{0}$, and multiplicities $r(n)$. Introducing in the theorem $(1.35)$ the spectral function $h((2 \pi / L) \varrho):=\phi\left(\varrho^{2}\right)$ with

- $h: \mathbb{R} \rightarrow \mathbb{C}$, continuous

- $h$ even i.e. $h(-p)=h(p)$

- $h(p)=O\left(\frac{1}{|p|^{2+\varepsilon}}\right),|p| \rightarrow \infty, \varepsilon>0$, 
we arrive at the trace formula on the torus $\mathbb{T}^{2}$

$$
\sum_{n=0}^{\infty} r(n) h\left(p_{n}\right)=\frac{|\Omega|}{2 \pi} \int_{0}^{\infty} p h(p) \mathrm{d} p+|\Omega| \sum_{n=1}^{\infty} r(n) \hat{h}(L \sqrt{n}),
$$

where $\hat{h}(x)$ denotes the Fourier-Bessel (or Hankel) transform of $h(p)$ :

$$
\hat{h}(x):=\frac{1}{2 \pi} \int_{0}^{\infty} p h(p) J_{0}(p x) d p .
$$

(In deriving the first term on the right-hand side of (1.37), we have used $r(0)=1=$ $J_{0}(0)$ and $L^{2}=|\Omega|$.) Note that the left-hand side of (1.37) can be written as the trace of the trace class operator

$$
h\left(\left(-\Delta_{\mathbb{T}^{2}}\right)^{1 / 2}\right): L^{2}(\Omega) \rightarrow L^{2}(\Omega)
$$

with

$$
h\left(\left(-\Delta_{\mathbb{T}^{2}}\right)^{1 / 2}\right) f=\sum_{m \in \mathbb{Z}^{2}} h\left(\sqrt{\lambda_{m}}\right)\left(e_{m} \mid f\right) e_{m}, \quad \text { for } f \in L^{2}(\Omega),
$$

i.e.

$$
\sum_{n=0}^{\infty} r(n) h\left(p_{n}\right)=\operatorname{Tr} h\left(\left(-\Delta_{\mathbb{T}^{2}}\right)^{1 / 2}\right)
$$

which explains why (1.37) is called a trace formula.

Due to the conditions (1.36) on the spectral function $h(p)$, the operator $h\left(\left(-\Delta_{\mathbb{T}^{2}}\right)^{1 / 2}\right)$ is actually a Hilbert-Schmidt operator with kernel $G_{h}(x, y)=\bar{G}_{h}(\gamma, x) \in$ $L^{2}(\Omega \times \Omega)$ satisfying, for $f \in L^{2}(\Omega)$,

$$
\left(h\left(\left(-\Delta_{\mathbb{T}^{2}}\right)^{1 / 2}\right) f\right)(x)=\int_{\Omega} G_{h}(x, \gamma) f(\gamma) \mathrm{d}^{2} \gamma .
$$

Furthermore, $G_{h}(x, \gamma)$ has the uniformly convergent expression in terms of the orthonormal eigenfunctions $e_{m} \in L^{2}(\Omega)$ (Mercer's theorem)

$$
G_{h}(x, y)=\sum_{m \in \mathbb{Z}^{2}} h\left(\sqrt{\lambda_{m}}\right) e_{m}(x) \bar{e}_{m}(\gamma)
$$

which expresses the fact that $e_{m}$ is an eigenfunction of the operator $h\left(\left(-\Delta_{\mathbb{T}^{2}}\right)^{1 / 2}\right)$ with eigenvalue $h\left(\sqrt{\lambda_{m}}\right)$. From this one immediately derives the pre-trace formula

$$
\operatorname{Tr} h\left(\left(-\Delta_{\mathbb{T}^{2}}\right)^{1 / 2}\right)=\int_{\Omega} G_{h}(x, x) \mathrm{d}^{2} x
$$


Pre-trace formulae of this type are the starting point for the derivation of trace formulae in the general case, for example in quantum mechanics, where the righthand side of (1.40) is expressed by the volume of the classical phase space and the classical actions evaluated along the periodic orbits of the corresponding classical system [63].

An alternative way to write the left-hand side of (1.37) is

$$
\sum_{n=0}^{\infty} r(n) h\left(p_{n}\right)=\int_{0}^{\infty} h(\sqrt{\lambda}) \mathrm{d} N(\lambda)
$$

where $N(\lambda)$ is the counting function, and the integral is understood as a Stieltjes integral. Rewriting in a similar way the first term on the right-hand side of (1.37)

$$
\frac{|\Omega|}{2 \pi} \int_{0}^{\infty} p h(p) \mathrm{d} p=: \int_{0}^{\infty} h(\sqrt{\lambda}) \mathrm{d} \bar{N}(\lambda),
$$

one obtains $\mathrm{d} \bar{N}(\lambda)=|\Omega| /(4 \pi) \mathrm{d} \lambda$ and thus, immediately, the smooth term

$$
\bar{N}(\lambda)=\frac{|\Omega|}{4 \pi} \lambda,
$$

which turns out to be exactly the leading Weyl term of $N(\lambda)$, see (1.4) and Section 1.3.6.

\section{3 .5}

\section{Spectral Geometry: Interpretation of the Trace Formula} on the Torus $\mathbb{T}^{2}$ in Terms of Periodic Orbits

While the left-hand side of the trace formula (1.37) has a simple spectral interpretation (being just the sum over the "frequencies" $p_{n}=\sqrt{\bar{\lambda}_{n}}$ of the eigenvibrations on $\mathbb{T}^{2}$, evaluated on a large class of spectral functions $h(p)$, see Equation (1.36)), the infinite series on the right-hand side has a simple geometrical interpretation as can be seen by rewriting (1.37) as follows

$$
\sum_{n=0}^{\infty} r(n) h\left(p_{n}\right)=|\Omega| \hat{h}(0)+|\Omega| \sum_{n=1}^{\infty} \sum_{k=1}^{\infty} r\left(k^{2} l_{n}^{2}\right) \hat{h}\left(k l_{n}\right) .
$$

Here $\left\{l_{n}\right\}_{n \in \mathbb{N}}$ denotes the primitive length spectrum on $\mathbb{T}^{2}$ with

$$
l_{n}=L \sqrt{m_{1}^{2}+m_{2}^{2}}=L \sqrt{n},
$$

where $n$ is a square-free integer with $r(n) \neq 0 . l_{n}$ is the geometrical length of a primitive periodic orbit (closed geodesic) of the classical geodesic flow on $\mathbb{T}^{2}$. The nonprimitive periodic orbits have lengths $k l_{n}, k \geq 2$, where $k$ counts the $k^{\text {th }}$ traversal of the corresponding primitive periodic orbit with length $l_{n}$. The trace formula (1.42) 
displays a beautiful relation in spectral geometry relating the spectrum of the Laplacian to the length spectrum of the geodesic flow. The torus $\mathbb{T}^{2}$ is a compact Riemann surface of genus 1 and Gaussian curvature $K=0$. A generalization to surfaces of higher genus is given by the famous Selberg trace formula $[45,46]$ which has been much studied in the field of quantum chaos (see for example [47,64-66]) and string theory (see for example $[67,68]$ ) and will be discussed in Section 1.4.4.

\subsection{6}

\section{The Trace of the Heat Kernel on $d$-Dimensional Tori and Weyl's Law}

The trace formula (1.37), respectively (1.42), has the typical structure of a trace formula and is in some sense a "meta formula" since it allows one to derive an infinite number of relations depending on the special choice of the spectral function $h(p)$ satisfying the conditions (1.36). As a first example, let us calculate the trace of the heat kernel, which is obtained for the choice $h(p)=e^{-p^{2} t}, t>0$. With

$$
\hat{h}(x)=\frac{1}{2 \pi} \int_{0}^{\infty} p e^{-p^{2} t} J_{0}(p x) \mathrm{d} p=\frac{1}{4 \pi t} e^{-x^{2} / 4 t}
$$

we get $(t>0)$

$$
\begin{aligned}
\Theta_{\mathbb{T}^{2}}(t) & :=\operatorname{Tr} e^{t \Delta_{\mathbb{T}^{2}}}=\sum_{n=0}^{\infty} r(n) e^{-\left(4 \pi^{2} /|\Omega|\right) n t}=\frac{|\Omega|}{4 \pi t}+\frac{|\Omega|}{4 \pi t} \sum_{n=1}^{\infty} r(n) e^{-(|\Omega| / 4) t n} \\
& =\frac{|\Omega|}{4 \pi t}+\frac{|\Omega|}{4 \pi t} \sum_{n=1}^{\infty} \sum_{k=1}^{\infty} r\left(k^{2} l_{n}^{2}\right) e^{-k^{2} l_{n}^{2} / 4 t} .
\end{aligned}
$$

For $t \rightarrow 0^{+}$one thus obtains the correct $|\Omega| /(4 \pi t)$-term (and no higher order terms of the type $\sum_{n=-1}^{\infty} a_{n} t^{n / 2}$ as occurring in the general case), which yields the correct Weyl term, and an exponentially small remainder term behaving as $O\left(t^{-1} e^{-L^{2} / 4 t}\right)$. It is thus seen that the Weyl term corresponds to the "zero-length contribution" in the periodic orbit sum i.e. to the term obtained for $l_{0}:=0$, while the exponential remainder term is determined by the shortest primitive periodic orbit on $\mathbb{T}^{2}$ having the length $l_{1}=L$. As to physical applications, let us point out that the function $\Theta_{\mathbb{T}^{2}}(t)$ is for $t \sim 1 / T$, where $T$ denotes absolute temperature, identical to the partition function in statistical mechanics, and thus the Weyl term determines the high-temperature limit of the corresponding thermodynamical system.

Note that the trace of the heat kernel rewritten as $f(q):=\sum_{n=0}^{\infty} r(n) q^{n}$ with $q=e^{i \pi \tau}$, $\tau=i(4 \pi /|\Omega|) t$, plays the role of a generating function of the arithmetic function $r(n) . f(q)$ was already introduced by Jacobi in 1829 who derived

$$
f(q)=\left(\sum_{m \in \mathbb{Z}} q^{m^{2}}\right)^{2}=\left(\theta_{3}(0 \mid \tau)\right)^{2}
$$


for $\operatorname{Im} \tau>0$ in terms of the elliptic theta function $\theta_{3}$. Using the transformation formula $\theta_{3}(0 \mid \tau)=(-i \tau)^{-1 / 2} \theta_{3}(0 \mid-1 / \tau)$ derived by Poisson in 1823, one obtains again relation (1.43).

It is not difficult to generalize the result (1.43) to $d$-dimensional flat tori $\mathbb{T}^{d}:=$ $\mathbb{R}^{d} / \Gamma$ with $\Gamma=(L \mathbb{Z})^{d}$. The translation group $\Gamma$ has a natural embedding as a lattice in $\mathbb{R}^{d}$. To $\Gamma$ there is associated a uniquely defined dual lattice $\Gamma^{*}$ (called a reciprocal lattice in physics): $\Gamma^{*}=\left\{\gamma^{*} \in \mathbb{R}^{n}: \gamma \cdot \gamma^{*} \in \mathbb{Z}\right.$ for all $\left.\gamma \in \Gamma\right\}$. With $\gamma=L n, n \in \mathbb{Z}^{d}$, $\gamma^{*}=1 / L m, m \in \mathbb{Z}^{d}$, the eigenvalues of $-\Delta_{\mathbb{T}^{d}}$ are given by $\left(\lambda_{\gamma^{*}}\right)_{\gamma^{*} \in \Gamma^{*}}=4 \pi^{2}\left\|\gamma^{*}\right\|^{2}$ with eigenvectors $\left(e_{\gamma^{*}}\right)_{\gamma^{*} \in \Gamma^{*}}=\left(e^{2 \pi i\left(\gamma^{*} \cdot x\right)}\right)$. Furthermore, the length spectrum of the classical periodic orbits on $\mathbb{T}^{d}$ is given by $(\|\gamma\|)_{\gamma \in \Gamma}$. Using the Poisson summation formula as in the case $d=2$, it is straightforward to derive a trace formula on $\mathbb{T}^{d}$ from which one obtains, for example, for the trace of the heat kernel $(t>0)$

$$
\begin{aligned}
\Theta_{\mathbb{T}^{d}}(t) & :=\operatorname{Tr} e^{t \Delta_{T^{d}}}=\sum_{\gamma^{*} \in \Gamma^{*}} e^{-4 \pi^{2}\left\|\gamma^{*}\right\|^{2} t}=\frac{|\Omega|}{(4 \pi t)^{d / 2}} \sum_{\gamma \in \Gamma} e^{-\|\gamma\|^{2} / 4 t} \\
& =\frac{|\Omega|}{(4 \pi t)^{d / 2}}+O\left(t^{-d / 2} e^{-L^{2} / 4 t}\right) \quad\left(t \rightarrow 0^{+}\right) .
\end{aligned}
$$

Here the first term on the right-hand side corresponding to the identity element $I \in \Gamma$ with $\|I\|=0$ yields via the Tauberian theorem of Karamata (see Theorem 1.1 in Section 1.6) Weyl's law for $\mathbb{T}^{d}(\lambda \rightarrow \infty)$

$$
N(\lambda)=\frac{|\Omega|}{(4 \pi)^{d / 2} \Gamma(1+d / 2)} \lambda^{d / 2}+O\left(\lambda^{d / 2}\right),
$$

but the trace formula yields, in addition, an exact expression for the remainder term in the same way as discussed in detail for $\mathbb{T}^{2}$ in Section 1.3.9 below.

The case $d=3$ has important applications in several fields. For example, in solid state physics, chemistry and crystallography, one identifies the lattice $\Gamma$ with the atomic structure of crystals. Furthermore, the reciprocal lattice $\Gamma^{*}$ is very useful in analyzing diffraction phenomena in light and neutron scattering off crystals. In cosmology it has been proposed that the spatial section of our Universe is given by a 3-torus whose fundamental domain is a cube with side length $L \simeq 5 \times 10^{26} \mathrm{~m} \simeq$ $5.6 \times 10^{10}$ light years [69].

Finally we would like to mention that the case $d=16$, i.e. the tori $\mathbb{R}^{16} / \mathbb{Z}^{16}$ have played an important role in the attempts to answer Kac's question [9], since it had already been noticed by John Milnor in 1964 that the tori $\mathbb{T}^{16}$ give examples of nonisometric compact manifolds with the same spectrum of the Laplacian [70]. The construction of these lattices for $d=16$ had already been found by Witt in 1941 [71]. 
1.3.7

\section{Going Beyond Weyl's Law: One can Hear the Periodic Orbits of the Geodesic Flow on the Torus $\mathbb{T}^{2}$}

Let us consider another admissible spectral function $h(p)$ in the trace formula (1.42) which is slightly more general than the one used in the previous section for the heat kernel:

$$
h(p):=J_{0}(p s) e^{-p^{2} t}, \quad s \in \mathbb{R}, t>0 .
$$

With

$$
\hat{h}(x)=\frac{1}{2 \pi} \int_{0}^{\infty} p J_{0}(p s) e^{-p^{2} t} J_{0}(p x) d p=\frac{1}{4 \pi t} e^{-\left(s^{2}+x^{2}\right) / 4 t} I_{0}\left(\frac{s x}{2 t}\right)
$$

( $I_{0}(z)$ is the modified Bessel function) we arrive at the trace formula $(s \in \mathbb{R}, t>0)$

$$
\begin{aligned}
G(s, t) & :=\operatorname{Tr}\left(J_{0}\left(s\left(-\Delta_{\mathbb{T}^{2}}\right)^{1 / 2}\right) e^{t \Delta_{\mathbb{T}^{2}}}\right)=\sum_{n=0}^{\infty} r(n) J_{0}\left(s \sqrt{\bar{\lambda}_{n}}\right) e^{-\bar{\lambda}_{n} t} \\
& =\frac{|\Omega|}{4 \pi t} e^{-s^{2} / 4 t}+\frac{|\Omega|}{4 \pi t} \sum_{n=1}^{\infty} \sum_{k=1}^{\infty} r\left(k^{2} l_{n}^{2}\right) e^{-\left(s^{2}+k^{2} l_{n}^{2}\right) / 4 t} I_{0}\left(\frac{s k l_{n}}{2 t}\right) .
\end{aligned}
$$

Since $I_{0}(0)=1$, it follows that (1.47) coincides in the limit $s \rightarrow 0$ with the trace of the heat kernel (1.43), $G(0, t)=\Theta_{\mathbb{T}^{2}}(t)$. Performing on the other hand for fixed $s>0$ the limit $t \rightarrow 0^{+}$i.e. eliminating the "regulator" $t$, one obtains the remarkable relation $(s>0)$

$$
G(s, 0)=\sum_{n=0}^{\infty} r(n) J_{0}\left(s \sqrt{\bar{\lambda}_{n}}\right)=\frac{|\Omega|}{2 \pi} \sum_{n=1}^{\infty} \sum_{k=1}^{\infty} \frac{r\left(k^{2} l_{n}^{2}\right)}{k l_{n}} \delta\left(s-k l_{n}\right),
$$

which is to be understood as an identity in the sense of distributions. Here we have used the asymptotic expansion (valid for $z \rightarrow+\infty$ )

$$
I_{0}(z)=\frac{1}{\sqrt{2 \pi z}} e^{z}\left(1+O\left(\frac{1}{z}\right)\right)
$$

and the delta-sequence

$$
\frac{1}{2 \sqrt{\pi t}} e^{-x^{2} / 4 t} \rightarrow \delta(x) \quad\left(t \rightarrow 0^{+}\right) .
$$

Relation (1.48) tells us that the formal trace $G(s, 0)=\operatorname{Tr} J_{0}\left(s\left(-\Delta_{\mathbb{T}_{2}}\right)^{1 / 2}\right)$ yields a welldefined distribution whose singular support is given for $s>0$ by

$$
\text { singsupp } G(s, 0)=\left\{k l_{n}\right\}, k \in \mathbb{N},
$$

i.e. by the primitive length spectrum $\left\{l_{n}\right\}$ of the geodesic flow on the torus and the nonprimitive length spectrum $\left\{k l_{n}\right\}, k \geq 2$. Thus the eigenvalues $\left\{\bar{\lambda}_{n}\right\}$ of the 
Laplacian on $\mathbb{T}^{2}$ together with their multiplicities $\{r(n)\}$ "know" the length spectrum of the closed geodesics of the classical motion on $\mathbb{T}^{2}$, i.e. one can hear the periodic orbits of the torus! Since the torus is uniquely given by its area $|\Omega|$ and its length spectrum $\left\{l_{n}\right\}$, we can conclude that the complete shape of the torus is audible.

A slightly different operator has been studied by Chazarain [72], Colin de Verdière [73, 74], and Duistermaat and Guillemin [75, 76], where the Bessel function $J_{0}$ is replaced by $\cos \left(s(-\Delta)^{1 / 2}\right)$ respectively $\exp \left(i s(-\Delta)^{1 / 2}\right)$.

\section{3 .8}

\section{The Spectral Zeta Function on the Torus $\mathbb{T}^{2}$}

Define for $s \in \mathbb{C}$, Re $s>1$, the spectral zeta function on $\mathbb{T}^{2}$ :

$$
\zeta_{\mathbb{T}^{2}}(s):=\operatorname{Tr}^{\prime}\left(-\Delta_{\mathbb{T}^{2}}\right)^{-s}=\sum_{n=1}^{\infty} \frac{r(n)}{\bar{\lambda}_{n}^{s}}=\frac{|\Omega|^{s}}{(2 \pi)^{2 s}} \sum_{n=1}^{\infty} \frac{r(n)}{n^{s}},
$$

where the prime at the trace denotes that the eigenvalue $\bar{\lambda}_{0}=0$ has been omitted. (Zeta functions of this type for general Laplace-Beltrami operators were introduced in $[77,78]$ following a suggestion of Weyl. See also [14].) With the help of

$$
\frac{1}{n^{s}}=\frac{1}{\Gamma(s)} \int_{0}^{\infty} \tau^{s-1} e^{-n \tau} \mathrm{d} \tau, \quad n>0, \operatorname{Re} s>0,
$$

we obtain for $\operatorname{Re} s>1$

$$
\Gamma(s) \zeta_{\mathbb{T}^{2}}(s)=\int_{0}^{1} t^{s-1}\left[\Theta_{\mathbb{T}^{2}}(t)-1\right] \mathrm{d} t+\int_{1}^{\infty} t^{s-1}\left[\Theta_{\mathbb{T}^{2}}(t)-1\right] \mathrm{d} t .
$$

Hence $\zeta_{\mathbb{T}^{2}}(s)$ is the Mellin transform of $\Theta_{\mathbb{T}^{2}}(t)$ with the eigenvalue zero omitted. Since

$$
\Theta_{\mathbb{T}^{2}}(t)=1+O\left(e^{-\left(4 \pi^{2} /|\Omega|\right) t}\right) \text { for } t \rightarrow \infty,
$$

the second integral has an analytic continuation to the whole complex s-plane as an entire function. Inserting in the first integral for $\Theta_{\mathbb{T}^{2}}(t)$ the expansion (1.43), we obtain for $\operatorname{Re} s>1$

$$
\zeta_{\mathbb{T}^{2}}(s)=\frac{|\Omega| /(4 \pi)}{s-1}+F(s),
$$

where $F(s)$ is an entire function. Thus we can extend the Dirichlet series (1.49) meromorphically to all $s \in \mathbb{C}$ having only one simple pole at $s=1$ with residue $|\Omega| /(4 \pi)$, which is given by the area of the torus. This pole is a direct consequence of the leading Weyl term in the expansion (1.43). It thus follows that the Dirichlet series $\sum_{n=1}^{\infty} r(n) / n^{s}$ diverges for $\operatorname{Re} s \leq 1$, but is convergent for $\operatorname{Re} s>1$, which will be important in the explicit formula for the remainder term in Weyl's law. Note that 
there exists the following closed expression, which can be considered as another generating function of $r(n)$ (see for example [79, pp. 265])

$$
\sum_{n=1}^{\infty} \frac{r(n)}{n^{s}}=4 \zeta(s) L(s)
$$

in terms of the Riemann zeta function $\zeta(s)$ and the Dirichlet $L$-series $L(s):=1-$ $1 / 3^{s}+1 / 5^{s}-\ldots$ with $L(1)=\pi / 4$, which has an entire extension.

The result (1.51) holds in general for a large class of eigenvalue problems; see for example reference [47] for the Laplace-Beltrami operator on compact Riemannian surfaces of genus $g \geq 2$. In the case of the Dirichlet Laplacian acting on a smooth bounded open set $\Omega \subset \mathbb{R}^{d}$ one can show [80] that $\xi_{\Omega}(s):=\operatorname{Tr}\left(-\Delta_{\Omega}^{D}\right)^{-s}$ possesses a meromorphic analytic continuation into the complex s-place with a leading simple pole at $s=d / 2$ and residue $|\Omega| /\left((4 \pi)^{d / 2} \Gamma(d / 2)\right)$. In particular, $s=0$ turns out to be a regular point such that the first derivative at $s=0, \zeta_{\Omega}^{\prime}(0)$, is well defined. This fact is then used to regularize the functional determinant of $-\Delta_{\Omega}^{D}$ by [80]

$$
\operatorname{det}\left(-\Delta_{\Omega}^{D}\right):=\exp \left(-\zeta_{\Omega}^{\prime}(0)\right)
$$

This method was introduced into physics by Stephen Hawking [81] as a convenient way to compute the determinants arising in the Feynman path integral approach to quantum field theory and quantum gravity. For applications of this method, see for example [82, pp. 37-43] in the case of quantum mechanics, and [67] in the case of string theory.

\section{3 .9}

\section{An Explicit Formula for the Remainder Term in Weyl's Law} on the Torus $\mathbb{T}^{2}$ and for the Circle Problem

To derive $N(\lambda)$ from the trace formula (1.37), we choose the function $h(p)=$ $\theta\left(\lambda-p^{2}\right), \lambda>0$. We then obtain with

$$
\frac{1}{2 \pi} \int_{0}^{\infty} p h(p) \mathrm{d} p=\frac{1}{2 \pi} \int_{0}^{\sqrt{\lambda}} p \mathrm{~d} p=\frac{\lambda}{4 \pi}
$$

and

$$
\hat{h}(x)=\frac{1}{2 \pi} \int_{0}^{\sqrt{\lambda}} p J_{0}(p x) \mathrm{d} p=\frac{\sqrt{\lambda}}{2 \pi x} J_{1}(\sqrt{\lambda} x)
$$

the relation

$$
N(\lambda)=\frac{|\Omega|}{4 \pi} \lambda+\frac{L}{2 \pi} \sqrt{\lambda} \sum_{n=1}^{\infty} \frac{r(n)}{\sqrt{n}} J_{1}(L \sqrt{n \lambda}) .
$$


This equation was found for the first time in Hardy's paper [54] who writes in a footnote: "The form of this equation was suggested to me by Mr. S. Ramanujan, ...". (As we shall see below, the sum in (1.52) is not absolutely convergent since the function $h(p)$ used in the derivation is not continuous. Relation (1.52) can be derived, however, by using an appropriate smoothing $[65,83]$.)

In order to study the asymptotic behavior of the remainder term, we employ the asymptotic formula

$$
J_{1}(x)=\sqrt{\frac{2}{\pi x}} \cos \left(x-\frac{3 \pi}{4}\right)+O\left(\frac{1}{x^{3 / 2}}\right) \quad(x \rightarrow \infty),
$$

and obtain in the limit $\lambda \rightarrow \infty$

$$
N_{\mathrm{fl}}(\lambda)=\lambda^{1 / 4} \frac{1}{\pi} \sqrt{\frac{L}{2 \pi}} \sum_{n=1}^{\infty} \frac{r(n)}{n^{3 / 4}} \cos \left(L \sqrt{\lambda n}-\frac{3 \pi}{4}\right)+O\left(\frac{1}{\lambda^{1 / 4}} \sum_{n=1}^{\infty} \frac{r(n)}{n^{5 / 4}}\right),
$$

where we have defined the "fluctuating part" of the counting function by $N_{\mathrm{fl}}(\lambda):=$ $N(\lambda)-(|\Omega| /(4 \pi)) \lambda . N_{\mathrm{fl}}(\lambda)$ describes the fluctuations of $N(\lambda)$ about the mean behavior $\bar{N}(\lambda):=(|\Omega| /(4 \pi)) \lambda$ given by Weyl's law, see (1.41). In Figure 1.1 we show a plot of $N(\lambda)$ for $L=2 \pi$ (which implies $N(\lambda)=v(\lambda)$ and $P(\lambda)=N_{\mathrm{fl}}(\lambda)$ for the remainder term in Gauss' circle problem) for small values of $\lambda \equiv x(0 \leq x \leq 50)$. Weyl's law is indicated as a straight line. One observes that the Weyl term does indeed describe the mean behavior of the staircase function $v(x)$ very well, even at small values of $x$. The fluctuating part $P(x)$ is shown in Figure 1.2 for small values $(0 \leq x \leq 50)$ and for large values $\left(10^{11} \leq x \leq 10^{11}+10^{7}\right)$ of $x$ and shows a very erratic behavior fluctuating about zero. In order to understand this behavior, one has to study the series in (1.53), which is a trigonometric series and therefore more difficult to control than a Fourier series. (Since $\sum_{1}^{\infty} r(n) / n^{5 / 4}<\infty$, see Section 1.3.8, the second term in (1.53) is bounded by $\lambda^{-1 / 4}$, and thus can be neglected.) Due to the divergence of the sum $\sum_{1}^{\infty} r(n) / n^{3 / 4}$, the trigonometric sum is only conditionally convergent, explaining the difficulty in proving Hardy's conjecture which amounts to the bound $O\left(\lambda^{\varepsilon}\right)$ for every $\varepsilon>0$ for this sum. (It is possible, however, to replace the sharp counting function $N(\lambda)$ by a smooth counting function depending on a smoothness parameter which leads to better convergence properties, see [65] and [83].)

In order to quantify the numerical observation that $N_{\mathrm{f}}(\lambda)$ oscillates about zero, let us calculate the mean value of $P(x)\left(=N_{\mathrm{fl}}(x)\right.$ for $\left.L=2 \pi\right)$ :

$$
\bar{P}(x):=\frac{1}{x} \int_{0}^{x} P(\gamma) \mathrm{d} y .
$$

We then obtain from (1.52) using

$$
\int_{0}^{x} \sqrt{y} J_{1}(2 \pi \sqrt{n y}) d y=\frac{x}{\pi \sqrt{n}} J_{2}(2 \pi \sqrt{n x})
$$




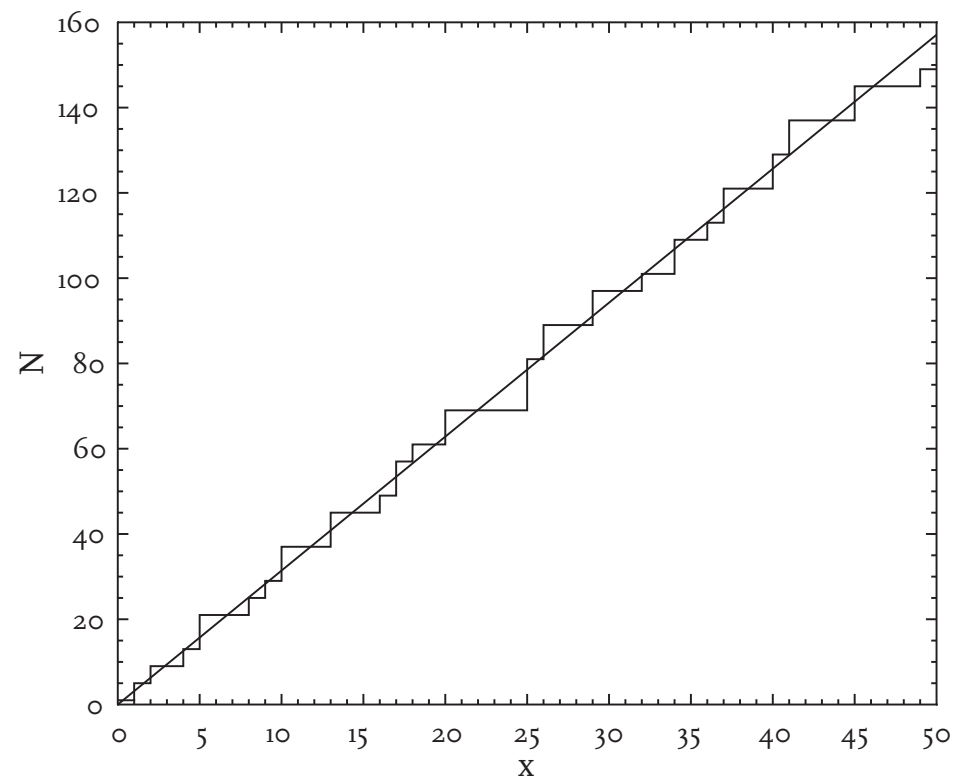

Figure 1.1 The counting function $v(x)$ for the Gaussian circle problem (respectively for a torus with $L=2 \pi$ ). The straight line shows the leading term $\pi x$ (Weyl's law).

and the asymptotics of the Bessel function $(x \rightarrow \infty)$

$$
\begin{aligned}
\bar{P}(x) & =\frac{1}{\pi} \sum_{n=1}^{\infty} \frac{r(n)}{n} J_{2}(2 \pi \sqrt{n x}) \\
& =\frac{x^{-1 / 4}}{\pi} \sum_{n=1}^{\infty} \frac{r(n)}{n^{5 / 4}} \cos \left(2 \pi \sqrt{n x}-\frac{5 \pi}{4}\right)+O\left(x^{-3 / 4}\right),
\end{aligned}
$$

which implies, since the sums in (1.54) are now absolutely convergent, $\lim _{x \rightarrow \infty}|\bar{P}(x)|=$ 0 [51, pp. 206]. A method to smooth possible spikes in $P(x)$, which originates in a paper by Cramér in 1922 [84], is to study higher moments of $P(x)$

$$
M_{k}(x):=\frac{1}{x} \int_{0}^{x}|P(y)|^{k} \mathrm{~d} \gamma
$$

for $k>0$ and

$$
m_{k}(x):=\frac{1}{x} \int_{0}^{x}(P(y))^{k} \mathrm{~d} y
$$

for $k=1,3,5, \ldots$ (Note that $\left.m_{1}(x)=\bar{P}(x)\right)$. The following results are known [85]

$$
\begin{array}{lll}
M_{k}(x) \rightarrow C_{k} x^{k / 4}, & k \in[0,9] & \\
m_{k}(x) \rightarrow c_{k} x^{k / 4}, & k=3,5,7,9 . & (x \rightarrow \infty)
\end{array}
$$



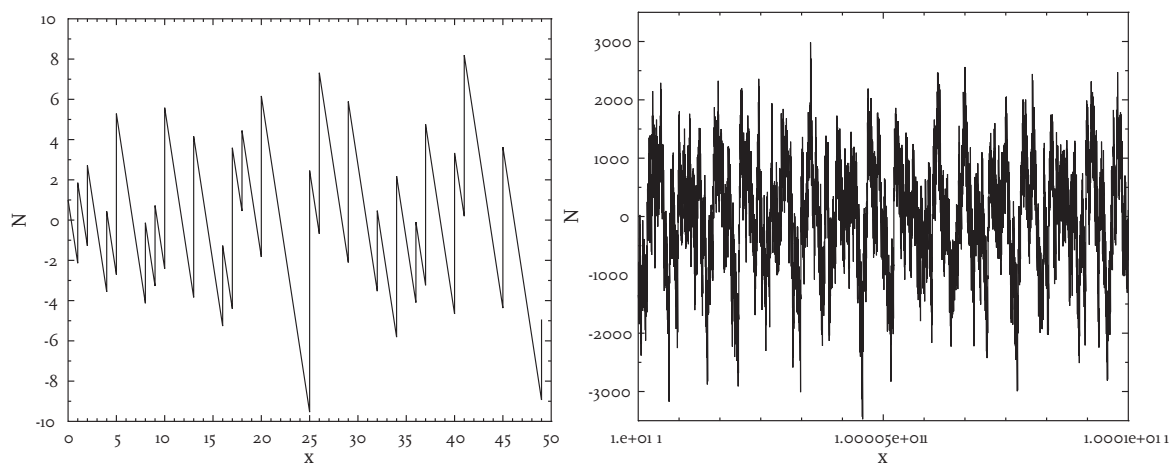

Figure 1.2 The remainder term $P(x)$ of the Gaussian circle problem (respectively the fluctuating part of the torus problem with $L=2 \pi$ ) is shown in different intervals.

$\left(C_{2}=1 /\left(3 \pi^{2}\right) \sum_{n=1}^{\infty} r(n)^{2} / n^{3 / 2}[84]\right)$. It follows that the moments (1.57) are consistent with Hardy's conjecture, $P(x)=O\left(x^{1 / 4+\varepsilon}\right)$, since this implies $\left(m_{k}(x)\right)^{1 / k}=O\left(x^{1 / 4}\right)$ resp. $\left(M_{k}(x)\right)^{1 / k}=O\left(x^{1 / 4}\right)$, but of course they do not prove it. Nevertheless it seems justified to say that the "mean" behavior of $P(x)$ is proportional to $x^{1 / 4}$ for $x \rightarrow \infty$.

\subsection{0}

\section{The Value Distribution of the Remainder Term in the Circle Problem}

In the preceding section we saw that the remainder term $P(x)$ in the circle problem (respectively the fluctuating part $N_{\mathrm{fl}}(\lambda)$ in Weyl's law for the torus problem) is a very irregular function fluctuating about zero (see Figures 1.1 and 1.2). It thus appears natural to consider $P(x)$ as a random function of $x$ and to study its statistical properties in the limit $x \rightarrow \infty$, like its moments as in Equations (1.55) and (1.56), its limit distribution (if it exists), correlations etc., rather than to estimate its magnitude, i.e. trying to prove Hardy's conjecture. Since the moment $M_{2}(x)$, see (1.55), is the variance of $P(x)$, an obvious quantity to study is the normalized remainder term

$$
W(x):=\frac{P(x)}{\sqrt{M_{2}(x)}} .
$$

Since $M_{2}(x) \rightarrow C_{2} \sqrt{x}$ for $x \rightarrow \infty$, it turns out to be convenient to consider the function

$$
F(p):=\frac{P\left(p^{2}\right)}{\sqrt{p}}=\frac{1}{\pi} \sum_{n=1}^{\infty} \frac{r(n)}{n^{3 / 4}} \cos \left(2 \pi \sqrt{n} p-\frac{3 \pi}{4}\right)+O\left(\frac{1}{p}\right) \quad(p \rightarrow \infty)
$$

as a function of $p:=\sqrt{x}>1$ and $F(p)=0$ for $p<1$. Obviously, $F(p)$ fluctuates about zero and its mean value vanishes asymptotically for $p \rightarrow \infty$, whereas Cramér's result [84] implies that the second moment of $F(p)$ exists. There now arise the following questions. i) Does $F(p)$, where $p$ is randomly chosen from the interval 
$\left[1, p_{m}\right]$, have for $p_{m} \rightarrow \infty$ a limit distribution $f(\alpha) \mathrm{d} \alpha$ with probability density $f(\alpha)$ ? ii) Assuming that $f(\alpha)$ exists, what is its form? In view of the erratic behavior of $P\left(p^{2}\right)$ and thus of $F(p)$, one may guess that the central limit theorem can be applied to $F(p)$ and thus $f(\alpha)$ should be a Gaussian.

The study of the distribution of $F(p)$ was initiated by Heath-Brown [85] who proved that $F(p)$ has indeed a distribution function $f(\alpha)$ in the sense that, for any interval $[a, b] \subset \mathbb{C}$ we have

$$
\lim _{p_{m} \rightarrow \infty} \frac{1}{p_{m}} \mu\left\{p \in\left[0, p_{m}\right]: F(p) \in[a, b]\right\}=\int_{a}^{b} f(\alpha) \mathrm{d} \alpha
$$

(here $\mu$ denotes the Lebesgue measure.) Moreover, he proved that $f(\alpha)$ can be extended to an entire function on $\mathbb{C}$ and decreases faster than polynomially on the real line as $|\alpha| \rightarrow \infty$.

The results of Heath-Brown were developed further by Bleher, Cheng, Dyson and Lebowitz [86] who proved

$$
\lim _{p_{m} \rightarrow \infty} \frac{1}{p_{m}} \int_{0}^{p_{m}} g(F(p)) \varrho\left(\frac{p}{p_{m}}\right) \mathrm{d} p=\int_{-\infty}^{\infty} g(\alpha) f(\alpha) \mathrm{d} \alpha
$$

for every piecewise continuous bounded function $g(x)$ on $\mathbb{R}$ and for an arbitrary probability density $\varrho(x) \geq 0$ on $[0,1]$. In addition, they showed that for every $\varepsilon>0$ there exists $\alpha_{0}=\alpha_{0}(\varepsilon)>0$ such that, on the real line $\alpha \in \mathbb{R}$, we have the upper bound

$$
0 \leq f(\alpha)<e^{-|\alpha|^{4-\varepsilon}}
$$

when $|\alpha|>\alpha_{0}$, and that the cumulative distribution $C(\alpha):=\int_{-\infty}^{\alpha} f\left(\alpha^{\prime}\right) \mathrm{d} \alpha^{\prime}$ satisfies for every $\alpha>\alpha_{0}$ the lower bound

$$
C(-\alpha), 1-C(\alpha)>e^{-\alpha^{4+\varepsilon}} \text {. }
$$

These results $[85,86]$ came as a great surprise since they imply that $f(\alpha)$ decreases for $|\alpha| \rightarrow \infty$ roughly as $e^{-\alpha^{4}}$ and thus faster than a Gaussian density! A numerical computation of $f(\alpha)$ is shown in Figure 1.3 and compared with a normal Gaussian distribution. The deviation from a Gaussian distribution is clearly visible; moreover, one observes that $f(\alpha)$ is skewed towards positive values of $\alpha$.

In the next section we shall formulate a conjecture which states that the nonGaussian behavior of $f(\alpha)$ has its origin in the fact that the circle problem can be related to the remainder term of Weyl's law for a quantum mechanical system whose corresponding classical system (i.e. the geodesic flow on a torus with $L=2 \pi$ ) is integrable and thus regular.

The proof of the properties (1.60)-(1.62) is based on the fact that $F(p)$ is an almost periodic function of Besicovitch class $B^{2}[86,87]$, which means

$$
\lim _{N \rightarrow \infty} \lim _{p_{m} \rightarrow \infty} \frac{1}{p_{m}} \int_{0}^{p_{m}}\left|F(p)-\frac{1}{\pi} \sum_{n=1}^{N} \frac{r(n)}{n^{3 / 4}} \cos \left(2 \pi \sqrt{n} p-\frac{3 \pi}{4}\right)\right|^{2} \mathrm{~d} p=0 .
$$




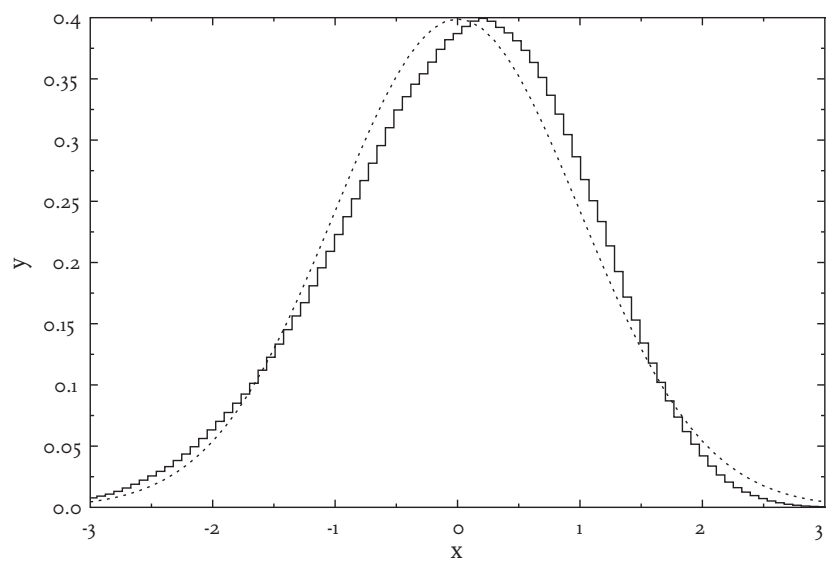

Figure 1.3 The distribution function $f(\alpha)$ is shown for the circle problem in comparison with a Gaussian normal distribution (dashed curve).

\subsubsection{1}

\section{A Conjecture on the Value Distribution of the Remainder Term in Weyl's Law for Integrable and Chaotic Systems}

In this section we would like to mention an application of Weyl's law in quantum mechanics. Let us consider a bound quantum system i.e. a system whose quantum Hamiltonian has a purely discrete energy spectrum $\left\{\lambda_{n}\right\}_{n \in \mathbb{N}}$. To have a specific example in mind, think of two-dimensional quantum billiards on a bounded domain $\Omega$ with area $|\Omega|$, for which the time-independent Schrödinger equation reads (in appropriate units) $-\Delta_{\Omega} u_{n}(x)=\lambda_{n} u_{n}(x)$ imposing (for example) Dirichlet or Neumann boundary conditions on $\partial \Omega$ (see (1.1) and (1.2)). Moreover, let us assume that Weyl's law holds in the form

$$
N(\lambda)=\bar{N}(\lambda)+N_{\mathrm{fl}}(\lambda),
$$

where the smooth part $\bar{N}(\lambda)$ describes asymptotically the mean behavior of the counting function $N(\lambda)$, i.e. the fluctuating remainder term $N_{\mathrm{fl}}(\lambda)$ satisfies

$$
\frac{1}{\lambda} \int_{\lambda_{1}}^{\lambda} N_{\mathrm{fl}}\left(\lambda^{\prime}\right) \mathrm{d} \lambda^{\prime} \rightarrow 0 \text { for } \lambda \rightarrow \infty .
$$

For "generic" two-dimensional billiards, there exists a three-term formula for $\bar{N}(\lambda)$

$$
\bar{N}(\lambda)=\frac{|\Omega|}{4 \pi} \lambda \mp \frac{|\partial \Omega|}{4 \pi} \sqrt{\lambda}+C,
$$

where the first two terms correspond to Weyl's conjecture (see (1.6) and the remarks after (1.7)), and the constant $C$ takes the curvature of $\partial \Omega$ and corner corrections into account (see (1.67)). 
The definition of what is meant by "generic" is a very subtle subject, the discussion of which is beyond the scope of this contribution. Examples of generic and nongeneric systems are discussed in [88]. A rigorous definition requires the introduction of geometrical concepts like "nonperiodicity" and "nonblocking"; see for example [89]. To derive the smoothed counting function $\bar{N}(\lambda)$, several averaging procedures have been invented, in particular by Brownell [90], which are described in [91]. For a simply connected domain $\Omega$ possessing piecewise smooth arcs of length $\gamma_{i}$ and corners of angle $\varphi_{j} \in(0,2 \pi]$ one obtains [91, p. 62] (1.66) with

$$
C=\frac{1}{12 \pi} \sum_{i} \int_{\gamma_{i}} \kappa(l) \mathrm{d} l+\frac{1}{24} \sum_{j}\left(\frac{\pi}{\varphi_{j}}-\frac{\varphi_{j}}{\pi}\right)
$$

where $\kappa(l)\left(l \in \operatorname{arc} \gamma_{i} \subset \partial \Omega\right)$ denotes the curvature of the arc $\gamma_{i}$. It should be noted, however, that the three-term formula (1.66) does not imply $N_{\mathrm{fl}}(\lambda)=O(1)$. On the contrary, the problem of determining $\alpha_{0}=\inf \alpha$ in the estimate $N_{\mathrm{fl}}(\lambda)=O\left(\lambda^{\alpha}\right)$ is a very difficult one; the circle problem discussed in Section 1.3.2 being an illustrative example.

To compare the quantal spectra of different systems, one has to get rid of the system-dependent constants in $\bar{N}(\lambda)$, which is achieved by "unfolding" the spectra by $x_{n}:=\bar{N}\left(\lambda_{n}\right)$. The unfolded spectrum $\left\{x_{n}\right\}_{n \in \mathbb{N}}$ has by construction a unit mean level spacing, and thus the corresponding counting function $\hat{N}(x):=\#\left\{x_{n} \leq x\right\}$ reads $\hat{N}(x)=x+\hat{N}_{f f}(x)$. Obviously,

$$
\frac{1}{x-x_{1}} \int_{x_{1}}^{x} \hat{N}_{f l}(y) d y \rightarrow 0 \quad \text { for } x \rightarrow \infty .
$$

In analogy to the approach discussed in Section 1.3.10 for the circle problem, we are interested in the statistical properties of the normalized remainder term

$$
W(x):=\frac{\hat{N}_{f l}(x)}{\sqrt{D(x)}}
$$

where $D(x)$ denotes the variance ( $\xi$ is a constant to be given below)

$$
D(x):=\frac{\xi}{x-x_{1}} \int_{x_{1}}^{x}\left(\hat{N}_{f f}(\gamma)\right)^{2} \mathrm{~d} \gamma
$$

We now consider $W(x)$ as a random variable, where $x$ is chosen randomly from the interval $\left[x_{1}, x_{m}\right]$ and ask whether $W(x)$ possesses in the limit $x_{m} \rightarrow \infty$ a limit distribution. If a limit distribution exists, it has by construction a second moment of one (if the second moment exists) and a first vanishing moment. 
We are now in a position to formulate the following

Conjencture 1.1 ([92, 93]) For bound conservative and scaling quantum systems the quantity $W(x)$, Equation (1.69), possesses for $x \rightarrow \infty$ a limit distribution with zero mean and unit variance. This distribution is absolutely continuous with respect to Lebesgue measure on the real line with a density $f(\alpha)$ i.e.

$$
\lim _{x_{m} \rightarrow \infty} \frac{1}{x_{m}} \int_{x_{1}}^{x_{m}} g(W(x)) \varrho\left(\frac{x}{x_{m}}\right) \mathrm{d} x=\int_{-\infty}^{\infty} g(\alpha) f(\alpha) \mathrm{d} \alpha,
$$

where $g(x)$ is a bounded continuous function on $\mathbb{R}$, and $\varrho(x) \geq 0$ a probability density on $[0,1]$. Furthermore,

$$
\int_{-\infty}^{\infty} \alpha f(\alpha) \mathrm{d} \alpha=0, \quad \int_{-\infty}^{\infty} \alpha^{2} f(\alpha) \mathrm{d} \alpha=1 .
$$

If the corresponding classical system is strongly chaotic, having only isolated and unstable periodic orbits, then $f(\alpha)$ is universally a Gaussian,

$$
f(\alpha)=\frac{1}{\sqrt{2 \pi}} e^{-\alpha^{2} / 2}
$$

In contrast, a classically integrable system leads to a system-dependent non-Gaussian density $f(\alpha)$.

Here a few remarks are in order. i) The normalization used in the definition (1.69) is crucial in order for a limit distribution to exist since in all interesting cases $D(x)$ diverges for $x \rightarrow \infty$. From Berry's [94] semiclassical analysis one obtains for generic integrable billiards

$$
D(x) \rightarrow c \sqrt{x}, \quad x \rightarrow \infty
$$

where $c$ is some nonuniversal constant. (For rigorous results, see the discussion of the torus billiard in Section 1.3.9 and [95]). In contrast, for generic classically chaotic systems one expects

$$
D(x) \rightarrow \frac{1}{2 \pi^{2} \beta} \ln x, \quad x \rightarrow \infty,
$$

with $\beta=1$ for systems with anti-unitary symmetry (for example time-reversal symmetry) and $\beta=2$ for systems without such a symmetry. ii) The constant $\xi$ in (1.70) takes the value $\xi=2 / 3$, if $D(x)$ obeys (1.74), and $\xi=1$ in the case of (1.75). iii) The conjecture is proven for some integrable systems like the torus (Gauss circle) problem, see [96] for a review. iv) The conjecture has been checked numerically for several integrable (like the isospectral billiard shown in Figure 1.5) and chaotic systems $[88,93]$ and has been found to hold with high statistical significance. v) In Figure 1.4 we show the numerical evaluation of $f(\alpha)$ for the strongly chaotic Hadamard-Gutzwiller model [64] which is the quantum version of the 
geodesic flow on a compact Riemann surface of genus two (for details, see Section 1.4). For this system there exists the rigorous Selberg trace formula [45] (see Equation (1.95) below) which yields for the remainder term $\hat{N}_{f}(x)$ the explicit expression (see (1.107) below)

$$
\hat{N}_{\mathrm{fl}}(x)=\frac{1}{\pi} \arg Z\left(\frac{1}{2}+i x\right)
$$

in terms of the Selberg zeta function $Z(s)$ evaluated on the critical line $s=1 / 2+i x$. For the numerical computation in Figure 1.4 we used the first 6000 eigenvalues with positive parity (computed by the boundary-element method [97]) of a generic (nonarithmetic) Riemann surface whose fundamental domain in the Poincaré-disk model for hyperbolic geometry is described in [97]. We conclude that the computed histogram is in nice agreement with the conjecture (1.73). vi) In many respects the nontrivial zeros of the Riemann zeta function $\zeta(s)$ behave like the scaled eigenvalues of a hypothetical classically chaotic system without anti-unitary symmetry, see Sections 1.4.8 and 1.4.9. The analogue of (1.76) reads

$$
\hat{N}_{\mathrm{fl}}(x)=\frac{1}{\pi} \arg \xi\left(\frac{1}{2}+i x\right)
$$

(see (1.108) below) counting only the zeros $\left\{x_{n}\right\}_{n \in \mathbb{N}}, \zeta\left(1 / 2+i x_{n}\right)=0$, with $\operatorname{Re} x_{n}>0$ and $-1 / 2<\operatorname{Im} x_{n}<1 / 2$. It has been shown by Selberg's moment method [98-100] that the corresponding quantity $W(x)$, with $D(x) \sim 1 / 2 \pi^{2} \ln \ln x$, has a Gaussian limit distribution in accordance with the conjecture. For a numerical calculation of $f(\alpha)$ using the first 50000 zeros and the 50000 zeros starting from the $10^{20}+$ 143780420 th zero, respectively, see Figure 8 in [101], which shows that the convergence of the probability distribution to the proven Gaussian limit distribution is very slow.

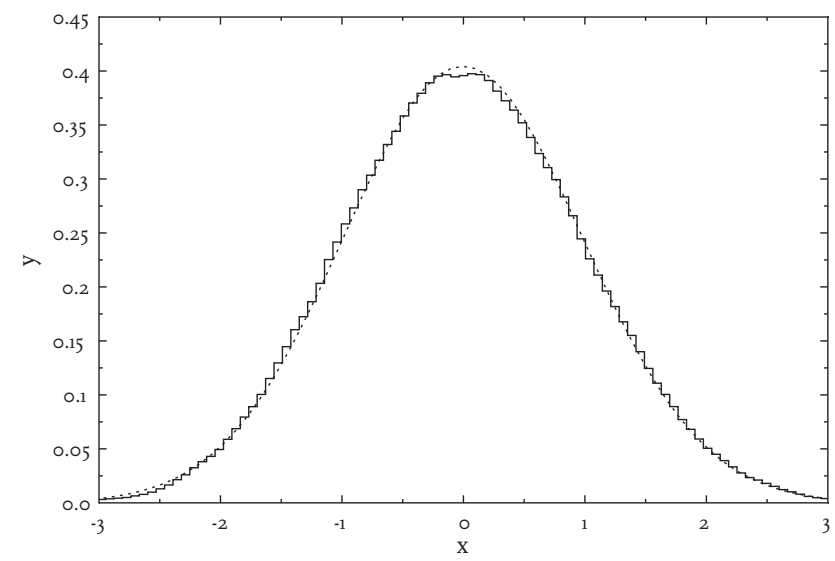

Figure 1.4 The distribution function $f(\alpha)$ is shown for the strongly chaotic Hadamard-Gutzwiller model in comparison with the conjectured Gaussian normal distribution (dashed curve). 


\section{4}

\section{Weyl's Law with Remainder Term. II}

\subsection{1 \\ The Laplace-Beltrami Operator on $d$-Dimensional Compact Riemann Manifolds $\mathcal{M}^{d}$ and the Pre-Trace Formula}

In many physical applications (ergodic theory, quantum mechanics, nonlinear optics, general relativity, string theory, and cosmology) one has to deal with the wave equation (or heat or Schrödinger equation) on non-Euclidean spaces. Important examples are $d$-dimensional manifolds or orbifolds $\mathcal{M}^{d}$ endowed with a Riemannian metric for which the Euclidean Laplacian has to be replaced by the corresponding Laplace-Beltrami operator. For simplicity, we discuss only manifolds with constant Gaussian curvature $K$.

Let us first consider smooth compact Riemannian manifolds $\mathcal{M}^{d}$ without boundary which are well studied and for which one can derive exact trace formulae and therefore can obtain full information on Weyl's law and even on Carleman's law $[102,103]$ involving the eigenfunctions. The simplest case of zero curvature $K=0$ i.e. flat tori $\mathcal{M}_{\Gamma}^{d}=\mathbb{R}^{d} / \Gamma$, where $\Gamma$ is a group of motions isomorphic to $\mathbb{Z}^{d}$, which are compact Riemannian manifolds, has already been discussed in Section 1.3 .

The case of homogeneous manifolds with constant positive curvature $K=+1$ is also well understood but will not be treated here.

The case of compact manifolds with constant negative curvature $K=-1$ and dimension $d \geq 2$ is highly nontrivial since the eigenvalues and eigenfunctions of the Laplace-Beltrami operator corresponding to the non-Euclidean (hyperbolic) metric are not known analytically. The geodesic flow i.e. the free motion of a point particle on these hyperbolic manifolds was already studied by Jacques Hadamard in $1898[104,105]$ and has played an important role in the development of ergodic theory ever since. Hadamard proved that all trajectories in this system are unstable and that neighboring trajectories diverge in time at an exponential rate, the most striking property of deterministic chaos. In 1980, Martin Gutzwiller drew attention to this system as a prototype example for quantum chaos [106]. Today the quantum system governed by the free Schrödinger equation i.e. the eigenvalue problem of the Laplace-Beltrami operator on these hyperbolic manifolds (or orbifolds), is known as the Hadamard-Gutzwiller model $[64,65,107]$. In dimension $d=3$, hyperbolic manifolds are possible candidates for the spatial section of the Universe and are investigated in cosmology [108].

In order to define a hyperbolic manifold, one considers Iso $\mathbb{H}^{d}$, the group of isometries on $\mathbb{H}^{d}$ (i.e. the distance-preserving bijections on $\mathbb{H}^{d}$ ), where $\mathbb{H}^{d}$ is the $d$-dimensional hyperbolic space. The action of an isometry $\gamma$ of $\mathbb{H}^{d}$ is denoted by $\gamma(z)$ with $z \in \mathbb{H}^{d}$. Take a discrete subgroup $\Gamma$ of Iso $\mathbb{H}^{d}$ and identify all points of $\mathbb{H}^{d}$ which can be transformed into each other by an element of $\Gamma$. Those points are called $\Gamma$-equivalent, and we put them into an equivalence class $\Gamma(z)=\{\gamma(z): \gamma \in \Gamma\}$ with $z \in \mathbb{H}^{d}$. The set of those classes defines the hyperbolic $d$-manifold represented 
by the quotient space $\mathcal{M}^{d}:=\mathbb{H}^{d} / \Gamma=\left\{\Gamma(z): z \in \mathbb{H}^{d}\right\}$. To visualize a given manifold, we have to take one representative from each class such that the set of all representatives yields a simply connected set in $\mathbb{H}^{d}$, called the fundamental domain $\Omega_{\Gamma}$. Here we discuss only compact manifolds whose fundamental domain is of finite volume, $\left|\Omega_{\Gamma}\right|<\infty$. One can cover all of $\mathbb{H}^{d}$ with $\Gamma$-translates of $\Omega_{\Gamma}$. This produces a tessellation of $\mathbb{H}^{d}$ in analogy to the case discussed in Section 1.3 for flat tori. The group $\Gamma$ is then called a hyperbolic crystallographic group or simply a hyperbolic lattice. The task is then to study the eigenvalue problem of the hyperbolic Laplacian $-\Delta u(z)=\lambda u(z), z \in \mathbb{H}^{d}, u \in L^{2}\left(\mathbb{H}^{d} / \Gamma, \chi\right)$, where $u$ is automorphic i.e. satisfies $u(\gamma(z))=\bar{\chi}(\gamma) u(z)$ for all $\gamma \in \Gamma$ and $z \in \mathbb{H}^{d}$. Here $\chi$ is any one-dimensional unitary representation of $\Gamma$, also called a character which satisfies $|\chi(\gamma)|^{2}=1$ for all $\gamma \in \Gamma$. Due to the compactness of $\mathcal{M}^{d}$, the spectrum of $-\Delta$ is discrete with $0=\lambda_{0}<\lambda_{1} \leq \lambda_{2} \leq \ldots$. (whether $\lambda_{0}=0$ exists depends on $\mathcal{M}^{d}$ ).

Let us consider the resolvent kernel $G_{\Gamma}\left(z, z^{\prime} ; \lambda\right)$ on $\mathbb{H}^{d} / \Gamma$ for $f \in L^{2}\left(\mathbb{H}^{d} / \Gamma, \chi\right)$

$$
\left[(-\Delta-\lambda)^{-1} f\right](z)=\int_{\Omega_{\Gamma}} G_{\Gamma}\left(z, z^{\prime} ; \lambda\right) f\left(z^{\prime}\right) \mathrm{d} \mu\left(z^{\prime}\right),
$$

where $\lambda \in \mathbb{C} \backslash[0, \infty)$. We then obtain the correlation function [107]

$$
C_{F}\left(z, z^{\prime}\right):=\sum_{n} F\left(\lambda_{n}\right) e_{n}(z) \bar{e}_{n}\left(z^{\prime}\right)=\frac{1}{\pi} \int_{0}^{\infty} F\left(\lambda^{\prime}\right) \operatorname{disc} G_{\Gamma}\left(z, z^{\prime} ; \lambda^{\prime}\right) \mathrm{d} \lambda^{\prime},
$$

where the spectral function $F(\lambda)$ is assumed to obey the following sufficient conditions:

- $F(\lambda)$ is holomorphic in a strip enclosing the positive real axis,

- $F(\lambda)$ drops faster than $\lambda^{-d / 2}$ for $\lambda \rightarrow \infty$.

The last condition is imposed to ensure convergence of the above expression for all $z, z^{\prime} \in \mathbb{H}^{d}$ including the diagonal $z=z^{\prime}$. For $z \neq z^{\prime}$ weaker conditions are sufficient. Furthermore, we have introduced the discontinuity of $G_{\Gamma}$ across the cut in the $\lambda$ plane

$$
\operatorname{disc} G_{\Gamma}\left(z, z^{\prime} ; \lambda\right):=\lim _{\varepsilon \rightarrow 0^{+}} \frac{1}{2 i}\left[G_{\Gamma}\left(z, z^{\prime} ; \lambda+i \varepsilon\right)-G_{\Gamma}\left(z, z^{\prime} ; \lambda-i \varepsilon\right)\right] .
$$

Since $C_{F}\left(z, z^{\prime}\right)$ is identical to the automorphic kernel of the operator $F(-\Delta)$, we obtain the pre-trace formula

$$
\sum_{n} F\left(\lambda_{n}\right)=\operatorname{Tr} F(-\Delta)=\int_{\Omega_{\Gamma}} C_{F}(z, z) \mathrm{d} \mu(z)
$$

\subsection{2}

The Sum Rule for the Automorphic Eigenfunctions on $\mathcal{M}^{d}$

In the next step, we make use of the alternative representation of the resolvent kernel which expresses the $\Gamma$-invariant kernel $G_{\Gamma}$ as a sum ("method of images") 
over the free resolvent kernel $G_{0}^{(d)}\left(z, z^{\prime} ; \lambda\right)$ on $\mathbb{H}^{d}$

$$
G_{\Gamma}\left(z, z^{\prime} ; \lambda\right)=\sum_{\gamma \in \Gamma} \chi(\gamma) G_{0}^{(d)}\left(z, \gamma\left(z^{\prime}\right) ; \lambda\right)
$$

The crucial point now is that $G_{0}^{(d)}$ is explicitly known for all $d \geq 2$, see [109]. Introduce the wave numbers $p_{n}$ via $p_{0}:=((d-1) / 2) i$ and $p_{n}:=\sqrt{\lambda_{n}-(d-1)^{2} / 4} \geq 0$ for $n \geq 1$. Here $p_{0}$ belongs to $\lambda_{0}=0$ (if it exists), and $p_{n}, n \geq 1$, to the eigenvalues $\lambda_{n} \geq(d-1)^{2} / 4$, where we have assumed that there are no so-called "small eigenvalues" with $0<\lambda_{n}<(d-1)^{2} / 4$. It is now convenient to replace the spectral function $F(\lambda)$ by a new spectral function

$$
h(p):=F\left(p^{2}+\frac{(d-1)^{2}}{4}\right)=F(\lambda): \mathbb{C} \rightarrow \mathbb{C},
$$

which has to fulfil the following sufficient conditions

- $h(-p)=h(p)$

- $h(p)$ is holomorphic in the strip $|\operatorname{Im} p| \leq \frac{d-1}{2}+\varepsilon, \varepsilon>0$

- $h(p)=O\left(p^{-d-\delta}\right), \delta>0$ for $|p| \rightarrow \infty$.

Then the correlation function takes the final form of $a$ "sum rule" for the automorphic eigenfunctions $e_{n}(d \geq 2)[107]$

$$
\sum_{n=0}^{\infty} h\left(p_{n}\right) e_{n}(z) \bar{e}_{n}\left(z^{\prime}\right)=\frac{2}{\pi} \sum_{\gamma \in \Gamma} \chi(\gamma) \int_{0}^{\infty} p h(p) \hat{\Phi}^{(d)}\left(\cosh d\left(z, \gamma\left(z^{\prime}\right)\right) ; p\right) d p,
$$

where $d\left(z, z^{\prime}\right)$ denotes the hyperbolic distance between arbitrary points $z, z^{\prime} \in \mathbb{H}^{d}$. $d\left(z, z^{\prime}\right)$ is a point-pair invariant, i.e. $d\left(\gamma(z), \gamma\left(z^{\prime}\right)\right)=d\left(z, z^{\prime}\right)$ for all $\gamma \in \Gamma$ and $z, z^{\prime} \in$ $\mathbb{H}^{d}$. For $z=z^{\prime}$ the distance $\tau_{\gamma}:=d(z, \gamma(z))$ is the length of a closed orbit, but which is in general not a periodic one. The function $\hat{\Phi}^{(d)}(\gamma ; p)$ is explicitly given by $(\gamma \geq 1)$

$$
\hat{\Phi}^{(d)}(\gamma ; p)=\frac{\pi}{(2 \pi)^{d / 2}} \frac{\left(\gamma^{2}-1\right)^{(2-d) / 4}}{2 p}\left|\frac{\Gamma(i p+(d-1) / 2)}{\Gamma(i p)}\right|^{2} P_{-1 / 2+i p}^{(2-d) / 2}(\gamma),
$$

where $P_{v}^{u}(\gamma)$ is the associated Legendre function of the first kind.

\subsection{3}

\section{Weyl's Law on $\mathcal{M}^{d}$ and its Generalization by Carleman}

At this point let us introduce the generalized counting function

$$
N_{\Gamma}^{(d)}\left(\lambda ; z, z^{\prime}\right):=\sum_{\lambda_{n} \leq \lambda} e_{n}(z) \bar{e}_{n}\left(z^{\prime}\right)
$$


which for $z=z^{\prime}$ gives Carleman's function $\sum_{\lambda_{n} \leq \lambda}\left|e_{n}(z)\right|^{2}[102,103]$ and after integrating over $\Omega_{\Gamma}$ the usual counting function

$$
N_{\Gamma}^{(d)}(\lambda)=\int_{\Omega_{\Gamma}} N_{\Gamma}^{(d)}(\lambda ; z, z) \mathrm{d} \mu(z)=\sum_{\lambda_{n} \leq \lambda} 1
$$

(since $\left.\int_{\Omega_{\Gamma}} e_{m}(z) \bar{e}_{n}(z) \mathrm{d} \mu(z)=\delta_{m n}\right)$.

We then obtain from the sum rule (1.80) the explicit formula

$$
\mathrm{d} N_{\Gamma}^{(d)}\left(\lambda ; z, z^{\prime}\right)=\mathrm{d} \bar{N}_{\Gamma}^{(d)}\left(\lambda ; z, z^{\prime}\right)+\mathrm{d} N_{\Gamma, \mathrm{f}}^{(d)}\left(\lambda ; z, z^{\prime}\right)
$$

with

$$
\mathrm{d} \bar{N}_{\Gamma}^{(d)}\left(\lambda ; z, z^{\prime}\right):=\frac{1}{\pi} \hat{\Phi}^{(d)}\left(\cosh d\left(z, z^{\prime}\right) ; \sqrt{\lambda-\left(\frac{d-1}{2}\right)^{2}}\right) \mathrm{d} \lambda
$$

and

$$
\mathrm{d} N_{\Gamma, \mathrm{f}}^{(d)}\left(\lambda ; z, z^{\prime}\right):=\frac{1}{\pi} \sum_{\gamma \in \Gamma^{\prime}} \chi(\gamma) \hat{\Phi}^{(d)}\left(\cosh d\left(z, \gamma\left(z^{\prime}\right)\right) ; \sqrt{\lambda-\left(\frac{d-1}{2}\right)^{2}}\right) \mathrm{d} \lambda
$$

where $\Gamma^{\prime}:=\Gamma \backslash\{I\}$ ( $I$ denotes the identity) and $\chi(I)=1$ was used. From our discussion of the trace formula for the tori $\mathbb{T}^{d}$ we expect that (1.85) gives the asymptotically leading smooth contribution to the generalized counting function (1.82). With $d(z, z)=0$ we obtain from $(1.85)$ for $z=z^{\prime}\left(p:=\sqrt{\lambda-((d-1) / 2)^{2}}\right)$

$$
\bar{N}_{\Gamma}^{(d)}(\lambda ; z, z):=\int_{((d-1) / 2)^{2}}^{\lambda} \mathrm{d} \bar{N}_{\Gamma}^{(d)}(\lambda ; z, z)=\frac{2}{\pi} \int_{0}^{p} \hat{\Phi}^{(d)}\left(1 ; p^{\prime}\right) p^{\prime} \mathrm{d} p^{\prime}
$$

which no longer depends on $z$ ! Here $\hat{\Phi}^{(d)}(1 ; p)$ follows from $(1.81)$

$$
\begin{aligned}
\hat{\Phi}^{(d)}(1 ; p) & =\frac{\pi}{(2 \pi)^{d / 2}} \frac{1}{2 p}\left|\frac{\Gamma(i p+(d-1) / 2)}{\Gamma(i p)}\right|^{2} \lim _{\gamma \rightarrow 1^{+}} \frac{P_{-1 / 2+i p}^{(2-d) / 2}\left(\gamma^{2}-1\right)^{(d-2) / 4}}{(4 \pi)^{d / 2} \Gamma(1+d / 2)} \cdot \frac{\pi}{2 p} \cdot\left|\frac{\Gamma(i p+(d-1) / 2)}{\Gamma(i p)}\right|^{2} \\
& =\frac{d}{(i p)}
\end{aligned}
$$

and thus

$$
\bar{N}_{\Gamma}^{(d)}(\lambda ; z, z)=\frac{d}{(4 \pi)^{d / 2} \Gamma(1+d / 2)} \int_{0}^{p}\left|\frac{\Gamma\left(i p^{\prime}+(d-1) / 2\right)}{\Gamma\left(i p^{\prime}\right)}\right|^{2} \mathrm{~d} p^{\prime}
$$


Using the asymptotic expansion

$$
\left|\frac{\Gamma(i p+(d-1) / 2)}{\Gamma(i p)}\right|^{2}=p^{d-1}\left(1+O\left(\frac{1}{p^{2}}\right)\right) \quad(p \rightarrow \infty),
$$

we immediately obtain

$$
\bar{N}_{\Gamma}^{(d)}(\lambda ; z, z)=\frac{1}{(4 \pi)^{d / 2} \Gamma(1+d / 2)} \lambda^{d / 2}+O\left(\lambda^{d / 2-1}\right) \quad(\lambda \rightarrow \infty)
$$

and after integration over $\Omega_{\Gamma}$ the non-Euclidean analog of Weyl's law $(d \geq 2)$

$$
\bar{N}_{\Gamma}^{(d)}(\lambda)=\frac{\left|\Omega_{\Gamma}\right|}{(4 \pi)^{d / 2} \Gamma(1+d / 2)} \lambda^{d / 2}+O\left(\lambda^{d / 2-1}\right) .
$$

Since one can show that the remainder term satisfies $N_{\Gamma, \mathrm{fl}}^{(d)}(\lambda ; z, z)=O\left(\lambda^{d / 2}\right)$, we obtain Carleman's law

$$
N_{\Gamma}^{(d)}(\lambda ; z, z)=\sum_{\lambda_{n} \leq \lambda}\left|e_{n}(z)\right|^{2}=\frac{\lambda^{d / 2}}{(4 \pi)^{d / 2} \Gamma(1+d / 2)}+O_{z}\left(\lambda^{d / 2}\right) \quad(\lambda \rightarrow \infty),
$$

which is a generalization of Weyl's law since it is not only a statement about the eigenvalues but also about the eigenfunctions. Note, however, that the sum rule (1.80) - being an exact explicit expression - contains much more information. To see this, let us consider the simplest case $d=2$ in more detail.

\subsection{4}

\section{The Selberg Trace Formula and Weyl's Law}

In the case $d=2$ we consider compact Riemann surfaces $\mathcal{M}^{2}=\mathbb{H}^{2} / \Gamma$ of genus $\mathrm{g} \geq 2$ with $\Gamma$ a strictly hyperbolic Fuchsian group of the first kind, $\Gamma \in \operatorname{PSL}(2, \mathbb{R})$. Such groups are characterized by the fact that all their group elements $\gamma$ (except the unity $I$ ) are hyperbolic. Here we choose for $\mathbb{H}^{2}$ the Poincaré!upper half plane $\mathbb{H}^{2}=\{z=x+i y: x, y \in \mathbb{R}, y>0\}$ with the hyperbolic metric

$$
\mathrm{d} s^{2}=\frac{\mathrm{d} x^{2}+\mathrm{d} y^{2}}{y^{2}}
$$

which is invariant under fractional linear transformations:

$$
z \rightarrow \gamma(z):=\frac{a z+b}{c z+d}
$$

where $a, b, c, d \in \mathbb{R}$ and $a d-b c=1$. Then the Laplace-Beltrami operator is $\Delta=$ $\gamma^{2}\left(\partial^{2} / \partial x^{2}+\partial^{2} / \partial y^{2}\right)$. It is also invariant under the group actions $\gamma \in \Gamma$. We then obtain from (1.81)

$$
\hat{\Phi}^{(2)}(\gamma ; p)=\frac{1}{4} \tanh (\pi p) P_{-1 / 2+i p}(\gamma)
$$


where $P_{v}(\gamma)$ denotes the Legendre function of the first kind.

Then the sum rule (1.80) takes the simple form $\left(\chi(\gamma)=1 \forall \gamma \in \Gamma, p_{0}=i / 2\right.$, $\left.p_{n}=\sqrt{\lambda_{n}-1 / 4} \geq 0, n \geq 1\right)[110]$

$$
\sum_{n=0}^{\infty} h\left(p_{n}\right) e_{n}(z) \bar{e}_{n}\left(z^{\prime}\right)=\frac{1}{2 \pi} \sum_{\gamma \in \Gamma} \hat{h}\left(\cosh d\left(z, \gamma\left(z^{\prime}\right)\right)\right)
$$

where the hyperbolic distance on $\mathbb{H}^{2}$ is given by

$$
\cosh d\left(z, z^{\prime}\right)=1+\frac{\left(x-x^{\prime}\right)^{2}+y^{2}+y^{\prime 2}}{2 \gamma y^{\prime}}
$$

Here $\hat{h}$ denotes the Mehler transform of the spectral function $h$ which is defined by the relations

$$
\begin{aligned}
& h(p)=\int_{1}^{\infty} \hat{h}(\gamma) P_{-1 / 2+i p}(\gamma) \mathrm{d} \gamma \\
& \hat{h}(\gamma)=\int_{0}^{\infty} p \tanh (\pi p) h(p) P_{-1 / 2+i p}(\gamma) \mathrm{d} p .
\end{aligned}
$$

In $[107,110]$ it was shown that the sum rule $(1.91)$ can be used to compute numerically the eigenfunctions $e_{n}(z)$ called nonholomorphic (or automorphic) forms or Maass waveforms, at least if the eigenvalues $\lambda_{n}$ are not too large. Taking the trace of the sum rule (1.91) one gets with (1.93) and $P_{-1 / 2+i p}(1)=1$ (the $\operatorname{SL}(2, \mathbb{R})$-invariant area element on $\mathbb{H}^{2}$ is $\left.\mathrm{d} \mu(z)=\mathrm{d} x \mathrm{~d} y / y^{2}\right)$

$$
\sum_{n=0}^{\infty} h\left(p_{n}\right)=\frac{\left|\Omega_{\Gamma}\right|}{2 \pi} \int_{0}^{\infty} p \tanh (\pi p) h(p) \mathrm{d} p+\frac{1}{2 \pi} \sum_{\gamma \in \Gamma^{\prime}} \int_{\Omega_{\Gamma}} \hat{h}(\cosh d(z, \gamma(z))) \mathrm{d} \mu(z)
$$

To evaluate the sum over $\gamma \in \Gamma^{\prime}$ involving the integral over $\hat{h}$ is a nontrivial task and was first achieved by Atle Selberg $[45,46]$ leading to the famous Selberg trace formula

$$
\sum_{n=0}^{\infty} h\left(p_{n}\right)=\frac{\left|\Omega_{\Gamma}\right|}{2 \pi} \int_{0}^{\infty} p \tanh (\pi p) h(p) \mathrm{d} p+\sum_{\{\gamma\}_{p}}^{\infty} \sum_{n=1}^{\infty} \frac{l(\gamma)}{2 \sinh (n l(\gamma) / 2)} \tilde{h}(n l(\gamma)),
$$

where $\tilde{h}(x)$ denotes the Fourier transform of $h(p)$

$$
\tilde{h}(x):=\frac{1}{2 \pi} \int_{-\infty}^{\infty} e^{i p x} h(p) \mathrm{d} p .
$$


The sum on the right-hand side of (1.95) runs over the length spectrum $\{l(\gamma)\}_{p}$ of the primitive periodic orbits of the geodesic flow on the surface $\mathcal{M}^{2}=\mathbb{H}^{2} / \Gamma$. Notice that the length spectrum is uniquely given by the conjugacy classes of the hyperbolic elements in $\Gamma$ as can be seen as follows. The elements $\gamma \in \Gamma$ of the discrete subgroups of $\operatorname{PSL}(2, \mathbb{R})$ can be represented as $2 \times 2$ matrices $\gamma=\left(\begin{array}{ll}a & b \\ c & d\end{array}\right)$ with real entries and $\operatorname{det} \gamma=a d-b c=1$. For a strictly hyperbolic group one has, for all $\gamma \neq \pm I$ : $|\operatorname{Tr} \gamma|=|a+d|>2$. The Jordan form of these matrices takes the form $\left(\begin{array}{cc}a & 0 \\ 0 & 1 / a\end{array}\right)$ with $|a|>1$, and the action of $\gamma$ gives $z \rightarrow \gamma(z)=a^{2} z$, where $N(\gamma):=a^{2}$ is called the norm of the element $\gamma$. Since there exists a unique relationship between the conjugacy classes in $\Gamma$ and the homotopy classes of closed paths on $\mathbb{H}^{2}$, one can define in each class a length $l(\gamma)$ by the length of the shortest path, and then obtains $N(\gamma)=e^{l(\gamma)}$, $l(\gamma)>0$. The length $l(\gamma)$ is then given by $\cosh (l(\gamma) / 2)=|\operatorname{Tr} \gamma| / 2$.

The sums and integrals in the Selberg trace formula are all absolutely convergent if the spectral function $h(p)$ satisfies conditions (1.79) for $d=2$. The Selberg trace formula (1.95) can be considered as a generalization and noncommutative analogue of the classical Poisson summation formula (1.33), respectively of the trace formulae (1.37) and (1.42-1.44) for flat tori.

From the Selberg trace formula (1.95) we can immediately read off the complete Weyl term of the counting function (see the discussion above for general $d \geq 2$ )

$$
\bar{N}_{\Gamma}^{\mathcal{M}^{2}}\left(p^{2}+\frac{1}{4}\right):=\frac{\left|\Omega_{\Gamma}\right|}{2 \pi} \int_{0}^{p} p^{\prime} \tanh \left(\pi p^{\prime}\right) \mathrm{d} p^{\prime}
$$

which behaves as

$$
\bar{N}_{\Gamma}^{\mathcal{M}^{2}}\left(p^{2}+\frac{1}{4}\right)=\frac{\left|\Omega_{\Gamma}\right|}{6} p^{3}+O\left(p^{5}\right) \quad \text { for } p \rightarrow 0
$$

and hence we obtain Weyl's law on compact Riemann surfaces of genus $g \geq 2$

$$
\bar{N}_{\Gamma}^{\mathcal{M}^{2}}\left(p^{2}+\frac{1}{4}\right)=\frac{\left|\Omega_{\Gamma}\right|}{4 \pi}\left(p^{2}-\frac{1}{12}\right)+O\left(p e^{-2 \pi p}\right) \text { for } p \rightarrow \infty .
$$

This asymptotic formula contains the standard Weyl term proportional to $\lambda$ and the volume $\left|\Omega_{\Gamma}\right|$, no term proportional to $\sqrt{\lambda}$, since $\mathcal{M}^{2}$ has no boundary, it has a constant term and then an exponentially small correction. Below we shall also derive the fluctuating remainder term of the counting function.

\section{4 .5}

\section{The Trace of the Heat Kernel on $\mathcal{M}^{2}$}

Choosing the spectral function $h(p)=e^{-\left(p^{2}+1 / 4\right) t}, t>0$, we obtain for the trace of the heat kernel on a compact Riemann surface $\mathcal{M}^{2}$ of genus $g \geq 2$ possessing the area 
$\left|\Omega_{\Gamma}\right|=4 \pi(g-1)$ (Gauss-Bonnet) the explicit formula $(t>0)$ [47]

$$
\begin{aligned}
\Theta^{\mathcal{H}^{2}}(t) & :=\sum_{n=0}^{\infty} e^{-\lambda_{n} t}=\sum_{n=0}^{\infty} e^{-\left(p_{n}^{2}+1 / 4\right) t}=\Theta_{1}^{\mathcal{M}^{2}}(t)+\Theta_{2}^{\mathcal{M}^{2}}(t), \\
\Theta_{1}^{\mathcal{M}^{2}}(t) & :=\left|\Omega_{\Gamma}\right| \frac{e^{-t / 4}}{(4 \pi t)^{3 / 2}} \int_{0}^{\infty} \frac{x}{\sinh (x / 2)} e^{-x^{2} / 4 t} \mathrm{~d} x \\
& =\frac{\left|\Omega_{\Gamma}\right|}{4 \pi t} \sum_{n=0}^{N} b_{n} t^{n}+O\left(t^{N}\right), \quad t \rightarrow 0^{+}, \\
b_{0} & =1, b_{n}=\frac{(-1)^{n}}{2^{2 n} n !}\left[1+2 \sum_{k=1}^{n}\left(\begin{array}{l}
n \\
k
\end{array}\right)\left(2^{2 k-1}-1\right)\left|B_{2 k}\right|\right], \quad n \in \mathbb{N}, \\
\Theta_{2}^{\mathcal{M}^{2}}(t) & :=\frac{e^{-t / 4}}{4 \sqrt{\pi t}} \sum_{\{\gamma\}_{p}} \sum_{n=1}^{\infty} \frac{l(\gamma)}{\sinh ((n l(\gamma)) / 2)} e^{-n^{2} l^{2}(\gamma) / 4 t},
\end{aligned}
$$

where $B_{2 k}$ are the Bernoulli numbers $\left(b_{1}=-1 / 3, b_{2}=1 / 15\right)$. This formula is the generalization of Poisson's transformation formula for the elliptic theta function $\theta_{3}$ discussed in Section 1.3.6 to Riemann surfaces of genus $g \geq 2$. Thus $\Theta^{\mathcal{M}^{2}}(t)$ can be called the non-Euclidean theta function. The formula (1.98) is quite interesting since it shows that for compact Riemann surfaces of genus $g \geq 2$ the complete small- $t$ asymptotics is explicitly known, see the term $\Theta_{1}^{\mathcal{M}^{2}}$, and not just the leading Weyl term $\left|\Omega_{\Gamma}\right| / 4 \pi t$. Furthermore, there even exists a closed expression for this contribution as an integral which is valid for all $t>0$ and is not just an asymptotic result in the limit $t \rightarrow 0^{+}$. Moreover, the remainder term $\Theta_{2}^{\mathcal{M}^{2}}$ also has an explicit representation as a sum over the length spectrum of periodic orbits. This term is exponentially small in the limit $t \rightarrow 0^{+}$and is determined by the shortest periodic orbit with primitive length $l\left(\gamma_{1}\right)>0$, i.e. $\Theta_{2}^{\mathcal{M}^{2}}(t)=O\left(t^{-1 / 2} e^{-l^{2}\left(\gamma_{1}\right) / 4 t}\right)$ in close analogy with the behavior on the torus $\mathbb{T}^{2}$.

\subsection{6}

\section{The Trace of the Resolvent on $\mathcal{M}^{2}$ and Selberg's Zeta Function}

In order to calculate the trace of the resolvent of $-\Delta$ on $\mathcal{M}^{2}$, one is led to substitute $h(p)=\left(1 / 4+p^{2}-\lambda\right)^{-1}$ in the trace formula. This function violates, however, the asymptotic condition in Equation (1.79) for $|p| \rightarrow \infty$, i.e. the resolvent is not of trace class as a consequence of Weyl's law which tells us that the eigenvalues behave as $\lambda_{n}=1 / 4+p_{n}^{2} \sim\left(4 \pi / \Omega_{\Gamma}\right) n$ for $n \rightarrow \infty$. Thus the resolvent has to be regularized properly. A very convenient regularization is given by the following choice. $(\operatorname{Re} s, \operatorname{Re} \sigma>1)$

$$
h(p)=\frac{1}{p^{2}+(s-1 / 2)^{2}}-\frac{1}{p^{2}+(\sigma-1 / 2)^{2}},
$$


which fulfills all the conditions (1.79) in the trace formula. For the integral (Weyl) term in the trace formula (1.95) one then obtains

$$
\frac{\left|\Omega_{\Gamma}\right|}{2 \pi} \int_{0}^{\infty} p \tanh (\pi p) h(p) \mathrm{d} p=-\frac{\left|\Omega_{\Gamma}\right|}{2 \pi}(\psi(s)-\psi(\sigma)),
$$

where $\psi(s):=\Gamma^{\prime}(s) / \Gamma(s)$ is the digamma function. Using the Fourier transform $(\operatorname{Re} s>1 / 2, x \geq 0)$

$$
\frac{1}{2 \pi} \int_{-\infty}^{\infty} \frac{e^{i p x}}{p^{2}+(s-1 / 2)^{2}} \mathrm{~d} p=\frac{1}{2 s-1} e^{-(s-1 / 2) x},
$$

the Selberg trace formula for the trace of the regularized resolvent reads $(\operatorname{Re} s, \operatorname{Re} \sigma>1)$

$$
\begin{aligned}
\sum_{n=0}^{\infty}\left(\frac{1}{\lambda_{n}+s(s-1)}-\frac{1}{\lambda_{n}+\sigma(\sigma-1)}\right)= & -\frac{\left|\Omega_{\Gamma}\right|}{2 \pi}(\psi(s)-\psi(\sigma)) \\
& +\frac{1}{2 s-1} A(s)-\frac{1}{2 \sigma-1} A(\sigma),
\end{aligned}
$$

where the function $A(s)$ is for $\operatorname{Re} s>1$ given by the absolutely convergent double sum

$$
A(s):=\sum_{\{\gamma\}_{p}} \sum_{n=1}^{\infty} \frac{l(\gamma) e^{-(s-1 / 2) n l(\gamma)}}{2 \sinh (n l(\gamma) / 2)} .
$$

It was one of Selberg's deep insights to realize that $A(s)$ can be rewritten for $\operatorname{Re} s>1$ as the logarithmic derivative of a kind of zeta function $Z(s)$ :

$$
\begin{aligned}
A(s) & =\sum_{\{\gamma\}_{p}} \sum_{n=1}^{\infty} \frac{l(\gamma) e^{-(s-1 / 2) n l(\gamma)}}{e^{n l(\gamma) / 2}-e^{-n l(\gamma) / 2}}=\sum_{\{\gamma\}_{p}} \sum_{n=1}^{\infty} \frac{l(\gamma) e^{-s n l(\gamma)}}{1-e^{-n l(\gamma)}} \\
& =\sum_{\{\gamma\}_{p}} \sum_{n=1}^{\infty} l(\gamma) e^{-s n l(\gamma)} \sum_{k=0}^{\infty} e^{-k n l(\gamma)}=\sum_{\{\gamma\}_{p}} \sum_{k=0}^{\infty} l(\gamma) \sum_{n=1}^{\infty} e^{-(s+k) n l(\gamma)} \\
& =\sum_{\{\gamma\}_{p}} \sum_{k=0}^{\infty} l(\gamma) \frac{e^{-(s+k) l(\gamma)}}{1-e^{-(s+k) l(\gamma)}}=\sum_{\{\gamma\}_{p}} \sum_{k=0}^{\infty} \frac{\mathrm{d}}{\mathrm{d} s} \ln \left(1-e^{-(s+k) l(\gamma)}\right) \\
& =\frac{\mathrm{d}}{\mathrm{d} s} \ln \left[\prod_{\{\gamma\}_{p}} \prod_{k=0}^{\infty}\left(1-e^{-(s+k) l(\gamma)}\right)\right]=: \frac{Z^{\prime}(s)}{Z(s)}
\end{aligned}
$$

Here we have defined the Selberg zeta function $(\operatorname{Re} s>1)$ [45]

$$
Z(s):=\prod_{\{\gamma\}_{p}}^{\infty} \prod_{k=0}^{\infty}\left(1-e^{-(s+k) l(\gamma)}\right),
$$


which is given as a generalized Euler product over the lengths of the primitive periodic orbits. It follows from Selberg's trace formula that the infinite products in (1.100) are absolutely convergent for Re $s>1$. Replacing $A(s)$ and $A(\sigma)$ in (1.99) by Selberg's zeta function, we obtain an exact relation [47] which expresses the trace of the regularized resolvent of $-\Delta$ on an arbitrary compact Riemann surface of genus $g \geq 2$ in terms of the well-known $\psi$-function and Selberg's zeta function. On the other hand, this relation allows us to prove that $Z(s)$ can be continued to the left of $\operatorname{Re} s=1$. This can be seen by rewriting (1.99) as follows [47]

$$
\begin{aligned}
& \frac{1}{2 s-1} \frac{Z^{\prime}(s)}{Z(s)}=-2(g-1) \psi(\sigma)+\left(\frac{1}{2 \sigma-1} \frac{Z^{\prime}(\sigma)}{Z(\sigma)}-\frac{1}{\sigma(\sigma-1)}\right) \\
& +2(g-1) \psi(s)+\frac{1}{s(s-1)}+\sum_{n=1}^{\infty}\left(\frac{1}{\lambda_{n}+s(s-1)}-\frac{1}{\lambda_{n}+\sigma(\sigma-1)}\right) .
\end{aligned}
$$

Note that the sum over the eigenvalues no longer contains the zero mode $\lambda_{0}=0$. Keeping the regulator $\sigma$ fixed with $\operatorname{Re} \sigma>1$, we see that the right-hand side of (1.101), derived for $\operatorname{Re} s>1$, is actually meromorphic for all $s \in \mathbb{C}$. Thus the left-hand side of (1.101) is also meromorphic, and so we obtain the analytic continuation of $Z(s)$ on $\mathbb{C}$. In fact, further inspection shows that the Selberg zeta function is an entire function of $s$ of order 2 whose "trivial" zeros are at $s=-k, k \in \mathbb{N}$, with multiplicity $2(g-1)(2 k+1)$. Furthermore, $s=1$ is a simple zero, and $s=0$ is a zero of multiplicity $2 g-1$. In addition $Z(s)$ can have a finite number of zeros on the real axis between 0 and 1 located at $s=1 / 2 \pm \sqrt{1 / 4-\lambda_{n}}$ corresponding to the so-called "small" eigenvalues $0<\lambda_{n}<1 / 4$. For surfaces of genus $g>2$, one has at most $4 g-3$ small eigenvalues $[111,112]$, while in the case of $g=2$ there is at most one small eigenvalue [113].

More importantly, $Z(s)$ has an infinite number of "nontrivial" zeros located at $s=$ $1 / 2 \pm i p_{n}, p_{n} \geq 0$, i.e. lying on the critical line $\operatorname{Re} s=1 / 2$, and thus one can say that the Riemann hypothesis is valid for $Z(s)$, a very remarkable result! One therefore has the exact quantization condition $\left(p_{n} \in \mathbb{R}\right)$

$$
z\left(\frac{1}{2}+i p_{n}\right)=0
$$

for the quantal eigenvalues $\lambda_{n}=p_{n}^{2}+1 / 4 \geq 1 / 4$ of the Schrödinger equation, which are completely determined by the lengths of the classical periodic orbits of the corresponding classical Hamiltonian system.

The reason behind the validity of the Riemann hypothesis in this case is obviously that $s(s-1)$ is an eigenvalue of a self-adjoint operator, and hence is real, whenever $s$ is a zero of $Z(s)$ within the critical strip. The question of whether something similar holds for the nontrivial zeros of the Riemann zeta function, will be discussed below.

The information on the zeros of $Z(s)$ enables us now to eliminate the regulator $\sigma$ in (1.101) by taking the limit $\sigma \rightarrow 1$. With $\psi(1)=-\gamma$, where $\gamma$ is Euler's constant, we define the generalized Euler constant $\gamma_{\Delta}$

$$
\gamma_{\Delta}:=2(g-1) \gamma+B
$$


with

$$
B:=\lim _{\sigma \rightarrow 1}\left(\frac{1}{2 \sigma-1} \frac{Z^{\prime}(\sigma)}{Z(\sigma)}-\frac{1}{\sigma(\sigma-1)}\right)=\frac{1}{2} \frac{Z^{\prime \prime}(1)}{Z^{\prime}(1)}-1 .
$$

Since $Z(s)$ possesses a simple zero at $s=1$, one has $Z^{\prime}(1) \neq 0$ (actually $Z^{\prime}(1)>0$ holds) and thus the constant $B$ is well defined. We then obtain for the trace of the regularized resolvent of $-\Delta$ on $\mathcal{M}^{2}=\mathbb{H}^{2} / \Gamma$ the final result [47]

$$
\frac{1}{s(s-1)}+\sum_{n=1}^{\infty}\left(\frac{1}{\lambda_{n}+s(s-1)}-\frac{1}{\lambda_{n}}\right)=\frac{1}{2 s-1} \frac{Z^{\prime}(s)}{Z(s)}-\gamma_{\Delta}-2(g-1) \psi(s) \text {. }
$$

1.4.7

The Functional Equation for Selberg's Zeta Function $Z(s)$

To derive the functional equation for $Z(s)$, we notice that $s(s-1)$ is invariant under $s \rightarrow 1-s$ and $2 s-1$ changes sign. If we then subtract (1.103) evaluated at $1-s$ from the same expression evaluated at $s$, we obtain

$$
\frac{1}{2 s-1} \frac{\mathrm{d}}{\mathrm{d} s} \ln \frac{Z(s)}{Z(1-s)}=2(g-1)(\psi(s)-\psi(1-s)) .
$$

Using the functional equation

$$
\psi\left(\frac{1}{2}+z\right)-\psi\left(\frac{1}{2}-z\right)=\pi \tan (\pi z)
$$

for the digamma function this then leads, with $z=s-1 / 2$, to the functional equation for $Z(s)$

$$
Z(s)=\exp \left(\left|\Omega_{\Gamma}\right| \int_{0}^{s-1 / 2} x \tan (\pi x) \mathrm{d} x\right) Z(1-s) .
$$

Evaluating the functional equation on the critical line i.e. choosing $s=1 / 2+i p$, $p \in \mathbb{R}$, we get

$$
Z\left(\frac{1}{2}+i p\right)=e^{-2 \pi i \bar{N}_{\Gamma}^{M^{2}}\left(p^{2}+1 / 4\right)} Z\left(\frac{1}{2}-i p\right)
$$

where the smooth term $\bar{N}_{\Gamma}^{\mathcal{M}^{2}}$ of the counting function given in (1.96) enters as a phase. It follows that the function

$$
\xi(p):=Z\left(\frac{1}{2}+i p\right) e^{i \pi \bar{N}_{\Gamma}^{\mathcal{M}^{2}}\left(p^{2}+1 / 4\right)}
$$

satisfies the simple functional equation $\xi(p)=\xi(-p)$, and furthermore that $\xi(p)$ is real if $p \in \mathbb{R}$, i.e. on the critical line. 
1.4.8

An Explicit Formula for the Remainder Term in Weyl's Law on $\mathcal{M}^{2}$ and the Hilbert-Polya Conjecture on the Riemann Zeros

Using the argument principle, one can derive the exact Weyl formula $\left(p \geq 0, p \neq p_{n}\right)$ for the counting function

$$
N_{\Gamma}^{\mathcal{M}^{2}}\left(p^{2}+\frac{1}{4}\right)=\bar{N}_{\Gamma}^{\mathcal{M}^{2}}\left(p^{2}+\frac{1}{4}\right)+\frac{1}{\pi} \arg Z\left(\frac{1}{2}+i p\right),
$$

which proves that the fluctuating term (remainder term) of the counting function is determined by the Selberg zeta function on the critical line

$$
N_{\Gamma, \mathrm{A}}^{\mathcal{M}^{2}}\left(p^{2}+\frac{1}{4}\right)=\frac{1}{\pi} \arg Z\left(\frac{1}{2}+i p\right) .
$$

The derivation of (1.106) is completely analogous to the well-known calculation leading to the counting function $N_{\mathrm{R}}(t)$ for the nontrivial Riemann zeros

$$
N_{\mathrm{R}}(t)=\bar{N}_{\mathrm{R}}(t)+\frac{1}{\pi} \arg \zeta\left(\frac{1}{2}+i t\right)
$$

which counts the number of zeros of the Riemann zeta function $\zeta(s)$ in the region $0<\operatorname{Re} s<1,0<\operatorname{Im} s \leq t$. Here the smooth term $\bar{N}_{\mathrm{R}}(t)$ is given by the famous Riemann-von Mangoldt formula [114]

$$
\bar{N}_{\mathrm{R}}(t)=\frac{t}{2 \pi} \ln t-\frac{1+\ln 2 \pi}{2 \pi} t+\frac{7}{8}+O\left(\frac{1}{t}\right) \quad(t \rightarrow \infty) .
$$

Note that Selberg introduced his zeta function $Z(s)$ around 1950 in analogy with the Riemann zeta function $\zeta(s)$ with the intention to shed some light on the properties of the nontrivial Riemann zeros and the Riemann hypothesis. He noticed the striking similarities between his trace formula (1.95) and the so-called explicit formulae in the theory of prime numbers [115], whose most general form is André Weil's explicit formula [116].

Weil's explicit formula establishes a deep relation between the nontrivial zeros $\varrho_{n}=1 / 2+i \tau_{n}, \tau_{n} \in \mathbb{C}$, of $\xi(s)$ and the prime numbers $p$ :

$$
\sum_{n=1}^{\infty} h\left(\tau_{n}\right)=\frac{1}{4 \pi} \int_{-\infty}^{\infty} \psi\left(\frac{1}{4}+i \frac{\tau}{2}\right) h(\tau) \mathrm{d} \tau+h\left(\frac{i}{2}\right)-\tilde{h}(0) \frac{\ln \pi}{2}-\sum_{p} \sum_{n=1}^{\infty} \frac{\ln p}{p^{n / 2}} \tilde{h}(n \ln p),
$$

where the "test function" $h(\tau)$ satisfies the same conditions (1.79) as the spectral function in the Selberg trace formula for $d=2$, and $\tilde{h}(x)$ is again its Fourier transform. Here the sum on the right-hand side runs over all primes $p$. Comparing Weil's formula (1.110) with Selberg's trace formula (1.95), one is tempted to interpret the nontrivial zeros of $\zeta(s)$ as eigenvalues of a hypothetical "Riemann operator" 
and the logarithm of the prime numbers as the "lengths" $l(p):=\ln p$ of the primitive "periodic orbits" of the corresponding hypothetical geodesic flow. The term on the right-hand side of (1.110) involving the summation over the primes then reads

$$
-\sum_{p} \sum_{n=1}^{\infty} \frac{l(p)}{e^{n l(p) / 2}} \tilde{h}(n l(p)),
$$

which is strikingly similar to the corresponding term in the Selberg trace formula (1.95) involving the summation over periodic orbits. Note, however, the difference between the denominator

$$
+2 \sinh \left(\frac{n l(\gamma)}{2}\right)=e^{n l(\gamma) / 2}-e^{-n l(\gamma) / 2}
$$

in (1.95) which has a dynamical interpretation in terms of the linearized Poincaré recurrence map for unstable hyperbolic periodic orbits, see for example [82,92], and the corresponding denominator $-e^{n l(p) / 2}$ in (1.111), for which no dynamical interpretation has been found until now; see, however, the paper by Alain Connes [117] who has devised a hermitian operator whose eigenvalues are the nontrivial Riemann zeros. His operator is the Perron-Frobenius operator (called the transfer operator in physics) of a classical dynamical system. In his framework he has found an explanation for the minus sign in (1.111).

At first sight it seems that there is another obstruction to the interpretation of the Riemann zeros as the eigenvalues of a dynamical system since the smooth counting function $\bar{N}_{\mathrm{R}}(t)$ (1.109) goes asymptotically as $\lambda /(2 \pi) \ln \lambda$, if we put $t=\lambda$, which differs from the standard behavior according to Weyl's law in dimension 2. It will be seen, however, in Section 1.5.2 that such logarithmic modifications to Weyl's law can occur, for example in membrane problems, for which the domain $\Omega$ is unbounded.

Mathematical wisdom has usually attributed the formulation of the idea of a hypothetical Riemann operator to Hilbert and Polya, independently, some time in the 1910s. (See Odlyzko's correspondence with Polya [118].)

There is another difference between the Riemann and the Selberg case. In the definition of $Z(s)$ in (1.100) one has a double product, whereas $\zeta(s)$ involves only a single one. Furthermore, the "Euler factor" occurs in $Z(s)$ with the $(+1)$ in the exponent, and in the case of $\zeta(s)$ with a $(-1)$. It turns out that, when one generalizes the Selberg zeta function to spaces of higher rank, the natural exponents are certain Euler characteristics which can take positive or negative values [119]. To get rid of the second product in (1.100), one simply considers the ratio

$$
R(s):=\frac{Z(s)}{Z(s+1)}=\prod_{\{\gamma\}_{p}}\left(1-e^{-s l(\gamma)}\right),
$$

and ends up with the Ruelle zeta function $R(s)$ [120], which is now a meromorphic function. $R(s)$ or rather $1 / R(s)$ has been discussed in terms of Beurling's generalized prime numbers and in connection with a generalized prime number theorem [121]. 
1.4.9

\section{The Prime Number Theorem vs. the Prime Geodesic Theorem on $\mathcal{M}^{2}$}

The famous prime number theorem states that the number of primes up to $x$, $\pi(x):=\#\{p: p \leq x\}$, is asymptotically equal to the logarithmic integral, given for $x>1$ by ( $f$ means the Cauchy principal value of the integral)

$$
\operatorname{li}(x):=\int_{0}^{x} \frac{\mathrm{d} t}{\ln t}=\frac{x}{\ln x}+\frac{x}{(\ln x)^{2}}+\ldots \quad(x \rightarrow \infty) .
$$

The fact that the density of primes near $x$ is about $1 / \ln x$ was already conjectured by Gauss in 1792 at the age of 15 . To derive a formula for $\pi(x)$ was Riemann's main goal in his famous paper from 1859, and it was for this purpose that he studied $\zeta(s)$ which had been introduced for integer argument already in 1735 by Euler who discovered among several other relations the formula $\zeta(2)=\pi^{2} / 6$ and in 1737 established the Euler product for $\zeta(m), m \geq 2$. The prime number theorem was proved in 1896 independently by Hadamard and de la Vallée Poussin by using the Riemann zeta function. It is worthwhile noticing that the first "elementary" proof was found by Selberg in 1949, see for example [46].

If one associates the prime numbers with the "lengths" $l(p):=\ln p$, the counting function $\mathcal{N}(l):=\#\{p: l(p) \leq l\}$ counts the number of hypothetical "periodic orbits" with length up to $l$. The prime number theorem is then converted into

$$
\mathcal{N}(l) \equiv \pi\left(e^{l}\right) \sim \operatorname{li}\left(e^{l}\right) \sim \frac{e^{l}}{l}, \quad(l \rightarrow \infty) .
$$

It is this result which gives perhaps the strongest support to the Hilbert-Polya conjecture, since it turns out that the counting function $\mathcal{N}_{\Gamma}^{\mathcal{M}^{2}}(l):=\#\{\gamma \in \Gamma: l(\gamma) \leq l\}$ of the genuine periodic orbits of the geodesic flow on $\mathcal{M}^{2}$ obeys Huber's law [122]

$$
\mathcal{N}_{\Gamma}^{\mathcal{M}^{2}}(l)=\operatorname{li}\left(e^{l}\right)+O\left(\frac{e^{(3 / 4) l}}{l}\right), \quad(l \rightarrow \infty) .
$$

This is a special case of the general prime geodesic theorem valid for the counting function of the lengths of the unstable periodic orbits of chaotic systems with a topological entropy $\tau>0$. In the general case, one has as leading term $e^{\tau l} / \tau l$. Thus Huber's law is consistent with the well-known fact that the geodesic flow on $\mathcal{M}^{2}$ is strongly chaotic, i.e. ergodic, mixing, possesses the Bernoulli property, and has topological entropy $\tau=1$. (Actually, all periodic orbits on $\mathcal{M}^{2}$ are unstable and possess the same Lyapunov exponent $\lambda(\gamma)=1$.)

Comparing (1.113) with (1.114), one concludes that the hypothetical dynamical system associated with the Riemann zeros should be chaotic, should have topological entropy $\tau=1$, and should possess a length spectrum of primitive periodic orbits exactly given by the logarithm of the primes, $l(p)=\ln p$ !

The validity of Huber's law (1.114) can be seen as follows. Due to the existence of the zero mode $\lambda_{0}=0$ with multiplicity one, $\Theta^{\mathcal{M}^{2}}(t)=1+O\left(e^{-\lambda_{1} t}\right), t \rightarrow \infty$, holds 
for the trace of the heat kernel on $\mathcal{M}^{2}$. Furthermore, one infers from (1.98) that the complete Weyl term $\Theta_{1}^{\mathcal{M}^{2}}(t)$ satisfies $\lim _{t \rightarrow \infty} \Theta_{1}^{\mathcal{M}^{2}}(t)=0$, and thus the remainder term $\Theta_{2}^{\mathcal{M}^{2}}(t)$ in (1.98) must satisfy $\lim _{t \rightarrow \infty} \Theta_{2}^{\mathcal{M}^{2}}(t)=1$. One therefore obtains the condition

$$
\lim _{t \rightarrow \infty} \frac{e^{-t / 4}}{2 \sqrt{\pi t}} \int_{l_{1}}^{\infty} l e^{-l^{2} / 4 t-l / 2} \mathrm{~d} \mathcal{N}_{\Gamma}^{\mathcal{M}^{2}}(l)=1,
$$

which yields $\mathrm{d} \mathcal{N}_{\Gamma}^{\mathcal{M}^{2}}(l)=e^{l} / l \mathrm{~d} l+\ldots$ for $l \rightarrow \infty$ in complete agreement with Huber's law (1.114).

In [123] an explicit formula for $\mathrm{dN}_{\Gamma}^{\mathcal{M}^{2}}(l)$ was derived including an oscillating remainder term. The derivation starts from Selberg's trace formula (1.95) and uses the Möbius inversion formula in complete analogy with Riemann's explicit formula for $\pi(x)$. The formula was used to compute the lowest part of the length spectrum for the most symmetric compact Riemann surface of genus $g=2$ using the first 200 eigenvalues, see Figure 1 in [123].

\section{5}

\section{Generalizations of Weyl's Law}

\subsection{1}

\section{Weyl's Law for Robin Boundary Conditions}

In Equations (1.66) and (1.67) we have given the three-term formula for the smooth term $\bar{N}(\lambda)$ for simply connected and bounded two-dimensional domains $\Omega$ with smooth boundary for Dirichlet as well as for Neumann boundary conditions. A generalization encountered in a nuclear physics context [124-126] are mixed or socalled Robin boundary conditions

$$
\alpha(x) u(x)+\partial_{n} u(x)=0 \quad(x \in \partial \Omega),
$$

which leaves the problem self-adjoint when $\alpha$ is real. The Dirichlet and Neumann boundary conditions are recovered in the limit $\alpha \rightarrow \infty$ and $\alpha \rightarrow 0$, respectively. For constant $\alpha \geq 0$ and excluding corners, Sieber et al. [127] derived the three-term Weyl formula

$$
\begin{aligned}
\bar{N}(\lambda)= & \frac{|\Omega|}{4 \pi} \lambda-\frac{|\partial \Omega|}{4 \pi}\left[1-2\left(\sqrt{1+\frac{\alpha^{2}}{\lambda}}-\frac{\alpha}{\sqrt{\lambda}}\right)\right] \sqrt{\lambda} \\
& +\left[1-3 \frac{\sqrt{\lambda}}{\alpha} \frac{\sqrt{1+\alpha^{2} / \lambda}-1}{\sqrt{1+\alpha^{2} / \lambda}}\right] \frac{1}{12 \pi} \int_{\partial \Omega} \kappa \mathrm{d} l .
\end{aligned}
$$

Since $\partial_{n} u=O(\sqrt{\lambda})$ in the limit $\lambda \rightarrow \infty$, the term $\partial_{n} u$ is asymptotically dominant in the boundary condition (1.115), and hence the mean spectrum will for fixed $\alpha$ always tend to the Neumann case. Therefore in the derivation of (1.116), $\lambda$ and $\alpha / \sqrt{\lambda}$ 
have been considered as independent parameters. One observes that the generalized Weyl law (1.116) interpolates neatly between the law (1.66), (1.67) for Dirichlet and Neumann boundary conditions. Formula (1.116) has been checked [127] in the case of the circle billiard, where $1 /(12 \pi) \int_{\partial \Omega} \kappa \mathrm{d} l=1 / 6$, for which the exact resolvent
kernel is known in closed form.

Apart from applications in nuclear physics, it was shown in [127] that the parametric dependence of the spectrum on the boundary condition is a very useful diagnostic tool in the analysis of spectra.

\subsection{2}

\section{Weyl's Law for Unbounded Quantum Billiards}

In Section 1.4.8 we have observed that the smooth term $\bar{N}_{\mathrm{R}}(\lambda)$ of the counting function of the nontrivial zeros of the Riemann zeta function grows asymptotically as $\lambda \ln \lambda$ which contradicts the classical eigenvalue asymptotics given by Weyl's law. Thus it appears that the interpretation of the nontrivial Riemann zeros as eigenvalues of the Laplacian is ruled out. It was pointed out, however, by Barry Simon $[128,129]$ that an asymptotic behavior of the form $\lambda \ln \lambda$ can occur for the eigenvalues of the two-dimensional Dirichlet Laplacian for certain unbounded regions which have a purely discrete spectrum. Since this nonclassical Weyl asymptotics again opens the possibility of identifying the nontrivial Riemann zeros with the eigenvalues of a hypothetical Riemann operator, it is important to determine also the nonleading terms of the counting function for such unbounded systems. As a representative example we here quote only the result for the so-called hyperbola billiard which is defined by the two-dimensional Euclidean Dirichlet Laplacian in the "horn-shaped" region

$$
\Omega=\left\{(x, y) \in \mathbb{R}_{+}^{2}: 0 \leq x \cdot y \leq 1\right\} .
$$

It was shown by Simon [128] that this quantum system possesses a purely discrete spectrum although the corresponding classical billiard has a continuous spectrum. In [130] the following asymptotic expansion for the trace of the heat kernel of the hyperbola billiard was derived $\left(t \rightarrow 0^{+}\right)$

$$
\Theta(t):=\operatorname{Tr} e^{t \Delta}=-\frac{\ln t}{4 \pi t}-\frac{a^{\prime}}{4 \pi t}+\frac{b}{8 \sqrt{\pi t}}+O\left(t^{-1 / 4}\right),
$$

where $a^{\prime}=2 \ln (2 \pi)-1-\gamma=2.0985 \ldots, b=4 \pi^{3 / 2} / \Gamma^{2}(1 / 4)=1.6944 \ldots$. Using the Karamata-Tauberian theorem in the form [129]: $\lim _{t \rightarrow 0^{+}}\left[-\left(t^{r} / \ln t\right) \operatorname{Tr} e^{t \Delta}\right]=c$ if and only if $\lim _{\lambda \rightarrow \infty}\left[\left(\lambda^{-r} / \ln \lambda\right) N(\lambda)\right]=c / \Gamma(r+1)$, one derives from (1.117) the leading term for the counting function

$$
N(\lambda)=\frac{1}{4 \pi} \lambda \ln \lambda+\ldots(\lambda \rightarrow \infty)
$$

To obtain the next terms one uses a theorem by Brownell [90] which allows to obtain a smoothed counting function $\bar{N}(\lambda)$. Form (1.117) one then obtains the mean 
asymptotic growth of the number of eigenvalues of the hyperbola billiard [130]

$$
\bar{N}(\lambda)=\frac{1}{4 \pi} \lambda \ln \lambda-\frac{a}{4 \pi} \lambda+\frac{b}{4 \pi} \sqrt{\lambda}+O\left(\lambda^{1 / 4} \ln \lambda\right) \quad(\lambda \rightarrow \infty),
$$

where $a=2(\ln (2 \pi)-\gamma)=2,5213 \ldots$. While the leading term in the last expression coincides with the first term of $\bar{N}_{\mathrm{R}}(\lambda)$, Equation (1.109), the second and third terms are different.

The hyperbola billiard has been extensively investigated in classical and quantum mechanics as a model for quantum chaos [131-133]. It turns out that the classical periodic orbits can be effectively enumerated using symbolic dynamics with a ternary code, and thus the length spectrum together with the Lyapunov exponents can be calculated with high precision. The topological entropy of this system is $\tau \approx 0.6$. Using the boundary-element method, a large number of eigenvalues could be calculated. The statistics of the eigenvalues is found to be consistent with the predictions of random matrix theory for the Gaussian orthogonal ensemble. Using the semiclassical Gutzwiller trace formula, one can define a dynamical zeta function defined by an Euler product over the classical periodic orbits in analogy with the Selberg zeta function (1.100). This zeta function satisfies an approximate functional equation and thus can be effectively used as a semiclassical quantization condition in analogy to the exact quantization condition (1.102).

\section{6}

\section{A Proof of Weyl's Formula}

Only for very special geometries of $\Omega$ is it possible to give an explicit formula for the eigenvalues of the Dirichlet Laplacian. Such a situation had been considered in the previous sections, another is given by rectangles and cubes. Weyl's original proof for Jordan measurable domains consisted in exhausting the domain by rectangles. This proof needs technical computations which we do not want to cover here. There is another more structured proof which uses properties of the heat equation and reveals an interesting connection between the heat kernel and the eigenvalues.

Let $\Omega \subset \mathbb{R}^{N}$ be open and bounded with boundary $\partial \Omega$. We want to impose a mild regularity condition on $\Omega$, namely we assume that for each $\varphi \in \mathrm{C}(\partial \Omega)$ the Dirichlet problem

$$
\left\{\begin{aligned}
u & \in \mathrm{C}(\bar{\Omega}) \cap \mathrm{C}^{2}(\Omega) \\
\Delta u & =0 \\
\left.u\right|_{\partial \Omega} & =\varphi
\end{aligned}\right.
$$

has a unique solution; i.e. we assume that $\Omega$ is Dirichlet regular. The Dirichlet problem is a classical subject of Potential Theory with physical interpretation in electrostatics.

There is a beautiful mathematical theory on the Dirichlet problem, and precise conditions on the boundary are known which imply Dirichlet regularity. It is a mild 
regularity condition on the boundary. If $\Omega$ has $\mathrm{C}^{1}$-boundary or if $\Omega$ is a polygon, then $\Omega$ is Dirichlet regular. More generally, Lipschitz continuity of the boundary suffices. In dimension 2 each simply connected domain (i.e. each open set without holes) is Dirichet regular.

Dirichlet regularity implies that all eigenfunctions of the Dirichlet Laplacian are continuous up to the boundary i.e. they lie in the space

$$
\mathrm{C}_{0}(\Omega):=\left\{u \in \mathrm{C}(\bar{\Omega}):\left.u\right|_{\partial \Omega}=0\right\} .
$$

Thus we may describe the Dirichlet Laplacian very simply by its spectral decomposition. We consider the Hilbert space $L^{2}(\Omega)$ with respect to the Lebesgue measure. Then there exists an orthonormal basis $\left\{e_{n}: n \in \mathbb{N}\right\}$ of $L^{2}(\Omega)$ such that

$$
\begin{aligned}
e_{n} & \in \mathrm{C}^{\infty}(\Omega) \cap \mathrm{C}_{0}(\Omega), \\
-\Delta e_{n} & =\lambda_{n} e_{n},
\end{aligned}
$$

where $0<\lambda_{1} \leq \lambda_{2} \leq \cdots \leq \lambda_{n} \rightarrow \infty$. We call $\lambda_{n}$ the $n^{\text {th }}$ eigenvalue of the Dirichlet Laplacian. Now Weyl's law says that

$$
\lim _{\lambda \rightarrow \infty} \frac{N(\lambda)}{\lambda^{N / 2}}=\frac{\omega_{N}}{(4 \pi)^{N / 2}}|\Omega|
$$

where $|\Omega|$ is the volume of $\Omega$ and $\omega_{N}=\pi^{N / 2} \Gamma(1+N / 2)$ is the volume of the unit ball in $\mathbb{R}^{N}$. By $N(\lambda)=\#\left\{n: \lambda_{n} \leq \lambda\right\}$ we denote the counting function.

For $f \in L^{2}(\Omega)$ we let

$$
\mathrm{e}^{t \Delta_{\Omega}^{\mathrm{D}}} f=\sum_{n=1}^{\infty} \mathrm{e}^{-\lambda_{n} t}\left(f \mid e_{n}\right) e_{n},
$$

where $(f \mid g)=\int_{\Omega} f g \mathrm{~d} x$ denotes the scalar product in $L^{2}(\Omega)$. Then $\mathrm{e}^{t \Delta_{\Omega}^{\mathrm{D}}}$ is a compact, self-adjoint operator on $L^{2}(\Omega)$. We call the family of operators $\left(\mathrm{e}^{t \Delta_{\Omega}^{\mathrm{D}}}\right)_{t \geq 0}$ the semigroup generated by the Dirichlet Laplacian. This semigroup is positive and dominated by the Gaussian semigroup $(G(t))_{t \geq 0}$, i.e. for $0 \leq f \in L^{2}(\Omega)$ we have

$$
0 \leq \mathrm{e}^{t \Delta_{\Omega}^{\mathrm{D}}} f \leq G(t) f, \quad(t>0)
$$

where

$$
\begin{aligned}
(G(t) f)(x) & :=\int_{\Omega} k_{t}^{0}(x, y) f(y) \mathrm{d} \gamma, \\
k_{t}^{0}(x, y) & :=(4 \pi t)^{-N / 2} \mathrm{e}^{-|x-y|^{2} / 4 t}, \\
|x-y|^{2} & :=\sum_{j=1}^{N}\left(x_{j}-\gamma_{j}\right)^{2}, \quad x, y \in \mathbb{R}^{N} .
\end{aligned}
$$

The domination property (1.121) implies also that $\mathrm{e}^{t \Delta_{\Omega}^{\mathrm{D}}}$ is defined by a measurable kernel $\tilde{k}_{t}(x, y)$ such that

$$
0 \leq \tilde{k}_{t}(x, y) \leq k_{t}^{0}(x, y) \quad \text { for all } x, y \in \Omega .
$$


We will express the kernel $\tilde{k}_{t}$ in terms of the eigenfunctions in (1.124). But here we recall that those operators $S$ on $L^{2}(\Omega)$ given by

$$
(S f)(x)=\int_{\Omega} q(x, y) f(\gamma) \mathrm{d} \gamma
$$

for some $q \in L^{2}(\Omega \times \Omega)$ are called Hilbert Schmidt operators. Such a Hilbert Schmidt operator $S$ is always compact. And if $S$ is self-adjoint, then its eigenvalues $\left(\mu_{n}\right)_{n \in \mathbb{N}}$ satisfy $\sum_{n=1}^{\infty} \mu_{n}^{2}<\infty$. Hence in our case

$$
\sum_{n=1}^{\infty} \mathrm{e}^{-2 t \lambda_{n}}<\infty \quad \text { for all } t>0 .
$$

Replacing $t$ by $t / 4$ we deduce that

$$
\sum_{n=1}^{\infty} \mathrm{e}^{-t \lambda_{n} / 2}<\infty \quad \text { for all } t>0 .
$$

Note that (1.122) implies that

$$
\left|\mathrm{e}^{-\lambda_{n} t} e_{n}\right|=\left|\mathrm{e}^{t \Delta_{\Omega}^{\mathrm{D}}} e_{n}\right| \leq G(t)\left|e_{n}\right|
$$

Since $\left\|e_{n}\right\|_{L^{2}}=1$, it follows from the Cauchy Schwarz inequality that

$$
\left(G(t)\left|e_{n}\right|\right)(x) \leq c t^{-N / 4} \text {, where } c=\pi^{-N / 4} 2^{-(3 / 4) N} .
$$

Thus

$$
\left|e_{n}(x)\right| \leq c t^{-N / 4} \mathrm{e}^{\lambda_{n} t}
$$

Letting $t=1 / \lambda_{n}$ we obtain

$$
\left|e_{n}(x)\right| \leq \tilde{c} \lambda_{n}^{N / 4} \quad(x \in \Omega, n \in \mathbb{N})
$$

where $\tilde{c}=c \cdot e$.

In view of (1.123), this estimate asserts that for each $t>0$, the series

$$
k_{t}(x, y):=\sum_{n=1}^{\infty} \mathrm{e}^{-\lambda_{n} t} e_{n}(x) e_{n}(\gamma)
$$

converges uniformly on the set $\bar{\Omega} \times \bar{\Omega}$ and defines a continuous, bounded function $k_{t}: \bar{\Omega} \times \bar{\Omega} \rightarrow \mathbb{R}$ such that $k_{t}(x, y)=0$ whenever $x \in \partial \Omega$ or $\gamma \in \partial \Omega$.

Note that

$$
\left(\mathrm{e}^{t \Delta_{\Omega}^{\mathrm{D}}} f\right)(x)=\int_{\Omega} k_{t}(x, \gamma) f(\gamma) \mathrm{d} \gamma,
$$

whenever $f \in\left\{e_{n}: n \in \mathbb{N}\right\}$. Since the $e_{n}$ form an orthonormal basis of $L^{2}(\Omega)$ it follows that (1.125) remains true for all $f \in L^{2}(\Omega)$. We have shown that the function $k_{t}$ is the kernel of the operator $\mathrm{e}^{t \Delta_{\Omega}^{\mathrm{D}}}$ i.e. $\widetilde{k}_{t}=k_{t}$. 
For our purposes the following immediate consequence is crucial.

$$
\int_{\Omega} k_{t}(x, x) \mathrm{d} x=\sum_{n=1}^{\infty} \mathrm{e}^{-\lambda_{n} t}
$$

This formula allows us to estimate the counting function $N(\lambda)=\#\left\{n: \lambda_{n}<\lambda\right\}$ with the help of the kernel $k_{t}$. For this we will make use of the following Tauberian theorem due to Karamata [134].

Theorem 1.1 Let $\left(\lambda_{n}\right)_{n \in \mathbb{N}}$ be a sequence of positive real numbers such that the series $\sum_{n \in \mathbb{N}} \mathrm{e}^{-\lambda_{n} t}$ converges for every $t>0$. Then for $r>0$ and $a \in \mathbb{R}$ the following are equivalent.

(a) $\lim _{t \rightarrow 0} t^{r} \sum_{n \in \mathbb{N}} \mathrm{e}^{-\lambda_{n} t}=a$

(b) $\lim _{\lambda \rightarrow \infty} \lambda^{-r} N(\lambda)=\frac{a}{\Gamma(r+1)}$

Here $N$ denotes the counting function $N(\lambda)=\#\left\{\lambda_{n} \leq \lambda\right\}$, and $\Gamma(r)=\int_{0}^{\infty} x^{r-1} \mathrm{e}^{-x} \mathrm{~d} x$ is the usual Gamma function.

Combining formula (1.126) and Theorem 1.1 we see that Weyl's law (1.119) is equivalent to the kernel estimate

$$
\lim _{t \rightarrow 0} t^{N / 2} \int_{\Omega} k_{t}(x, x) \mathrm{d} x=\frac{|\Omega|}{(4 \pi)^{N / 2}} .
$$

It is easily seen that the left-hand side of (1.127) is not greater than the right-hand side as the kernel $k_{t}$ is bounded by the Gaussian kernel i. e. $k_{t}(x, \gamma) \leq k_{t}^{0}(x, y)$ for $x, y \in \Omega, t>0$.

The lower estimate is more delicate. For this we will consider the heat equation on the infinite cylinder $\mathbb{R}_{+} \times \bar{\Omega}$ whose boundary we denote by $\Gamma=(\{0\} \times \bar{\Omega}) \cup$ $((0, \infty) \times \partial \Omega)$. It is a remarkable fact that Dirichlet regularity of $\Omega$ also implies that the following boundary value problem for the heat equation is well-posed.

Theorem 1.2 ([135, Theorem 6.2.8], [136]) Let $\psi \in \mathrm{C}(\Gamma)$. Then there exists a unique solution of

$$
\left\{\begin{aligned}
u & \in \mathrm{C}\left(\mathbb{R}_{+} \times \bar{\Omega}\right) \cap \mathrm{C}^{\infty}((0, \infty) \times \Omega), \\
\frac{\partial}{\partial t} u(t, x) & =\Delta u(t, x), \quad(t>0, x \in \Omega) \\
\left.u\right|_{\Gamma} & =\psi
\end{aligned}\right.
$$

This solution satisfies the parabolic maximum principle, which says that for all $t>0$ and all $0 \leq s \leq t, x \in \bar{\Omega}$,

$$
u(s, x) \leq \max _{\Gamma_{t}} u
$$

where $\Gamma_{t}:=\Gamma \cap([0, t] \times \bar{\Omega})$. 
Example 1.1 Let $f \in \mathrm{C}_{0}(\Omega)$ and define $\psi \in \mathrm{C}(\Gamma)$ by $\psi(0, x)=f(x)$ for $x \in \bar{\Omega}$, and $\psi(t, z)=0$ for $t>0, z \in \partial \Omega$. Then the solution of $(1.128)$ is given by $u(t, x)=\left(\mathrm{e}^{t \Delta_{\Omega}^{\mathrm{D}}} f\right)(x)$. Thus, the semigroup $\left(\mathrm{e}^{t \Delta_{\Omega}^{\mathrm{D}}}\right)_{t \geq 0}$ governs the homogeneous boundary value problem (1.128). Its solution can be expressed by the kernel $k_{t}$, namely,

$$
u(t, x)=\int_{\Omega} k_{t}(x, \gamma) f(\gamma) \mathrm{d} \gamma .
$$

For this reason we call $k_{t}$ the heat kernel associated with the Dirichlet Laplacian.

To obtain a lower bound for the kernel we formalize the idea that at some distance away from the boundary, $k_{t}$ behaves just like the Gaussian kernel.

Lemma 1.1 Let $x \in \Omega$ be arbitrary, and for $\gamma \in \Omega$ let $t_{0}(\gamma):=\operatorname{dist}(\gamma, \partial \Omega)^{2} / 2 N$ denote the scaled squared distance of $y$ to the boundary of $\Omega$. Then

$$
k_{t}^{0}(x, \gamma)-k_{t}(x, \gamma) \leq \begin{cases}(4 \pi t)^{-N / 2} \mathrm{e}^{-\operatorname{dist}(\gamma, \partial \Omega)^{2} / 4 t}, & t \leq t_{0}(\gamma), \\ \left(4 \pi t_{0}(\gamma)\right)^{-N / 2} \mathrm{e}^{-N / 2}, & t>t_{0}(\gamma) .\end{cases}
$$

Proof Fix $y \in \Omega$. Then by Theorem 1.2 there exists a unique function $p(\cdot, \cdot, \gamma)$ solving the parabolic boundary value problem

$$
\left\{\begin{aligned}
p(\cdot, \cdot, y) & \in \mathrm{C}\left(\mathbb{R}_{+} \times \bar{\Omega}\right) \cap \mathrm{C}^{\infty}((0, \infty) \times \Omega), & & \\
\frac{\partial}{\partial t} p(t, x, y) & =\Delta_{x} p(t, x, y), & & (t>0, x \in \Omega) \\
p(t, x, y) & =0, & & (x \in \bar{\Omega}) \\
p(t, x, y) & =(4 \pi t)^{-N / 2} \mathrm{e}^{-|x-y|^{2} / 4 t} . & & (t>0, x \in \partial \Omega)
\end{aligned}\right.
$$

Then $p(t, x, y)=k_{t}^{0}(x, y)-k_{t}(x, y)$. In fact, let $f \in \mathrm{C}_{0}(\Omega)$ be arbitrary, and let

$$
u(t, x):=\int_{\Omega}\left(k_{t}^{0}(x, y) f(y)-p(t, x, y) f(\gamma)\right) \mathrm{d} y .
$$

The properties $u \in \mathrm{C}^{\infty}((0, \infty) \times \Omega), u_{t}=\Delta u$ on $(0, \infty) \times \Omega$ and $u(t, x)=0$ if $x \in \partial \Omega$, $t>0$ are obvious. Moreover, it is easy to prove that $u$ can be continuously extended to $t=0$ and $u(0, x)=f(x)$ for all $x \in \bar{\Omega}$. Thus $u(t, \cdot)=\mathrm{e}^{t \Delta_{\Omega}^{\mathrm{D}}} f$ according to Example 1.1. Since $p$ solves a parabolic problem, we can use the parabolic maximum principle to deduce that $p$ attains its maximum on the boundary i. e.

$$
p(t, x) \leq \sup _{\substack{0 \leq s \leq t \\ x \in \partial \Omega \Omega}}(4 \pi s)^{-N / 2} \mathrm{e}^{-|x-y|^{2} / 4 s} \leq \sup _{0 \leq s \leq t}(4 \pi s)^{-N / 2} \mathrm{e}^{-\operatorname{dist}(y, \partial \Omega)^{2} / 4 s} .
$$

Calculating the derivative of $(4 \pi t)^{-N / 2} \mathrm{e}^{-\operatorname{dist}(\gamma, \partial \Omega)^{2} / 4 t}$ as a function in the variable $t$ one sees that the maximum is attained at time $t=t_{0}(\gamma)$. We can thus simplify (1.129) accordingly which completes the proof. 
We are interested in the error $\int_{\Omega}\left(k_{t}^{0}(x, x)-k(x, x)\right) \mathrm{d} x$ of the approximation of $k_{t}^{0}$ by $k_{t}$ as $t$ tends to 0 . Since the lemma essentially says that problems may only arise near the boundary, it is natural to decompose $\Omega$ into a good part $\Omega_{1}(t):=$ $\left\{x \in \Omega: \operatorname{dist}(x, \partial \Omega) \geq t^{1 / 4}\right\}$ and a bad part $\Omega_{2}(t):=\Omega \backslash \Omega_{1}(t)$. Note that $\left|\Omega_{2}(t)\right| \rightarrow 0$ as $t \rightarrow 0$. If $t \leq 1 / 4 N^{2}$, then for every $x \in \Omega_{1}(t)$ we have $t_{0}(x) \geq \sqrt{t} / 2 N \geq t$. Hence we can apply the lemma to obtain

$$
t^{N / 2} \int_{\Omega_{1}(t)}\left(k_{t}^{0}(x, x)-k_{t}(x, x)\right) \mathrm{d} x \leq|\Omega|(4 \pi)^{-N / 2} \mathrm{e}^{-\sqrt{t} / 4 t} \rightarrow 0 \quad(t \rightarrow 0) .
$$

On the other hand, using the trivial estimate $k_{t} \geq 0$ we see

$$
t^{N / 2} \int_{\Omega_{2}(t)}\left(k_{t}^{0}(x, x)-k_{t}(x, x)\right) \mathrm{d} x \leq\left|\Omega_{2}(t)\right|(4 \pi)^{-N / 2} \rightarrow 0 \quad(t \rightarrow 0) .
$$

Combining these two estimates, we have proved

$$
\liminf _{t \rightarrow 0} t^{N / 2} \int_{\Omega} k_{t}(x, x) \mathrm{d} x \geq \liminf _{t \rightarrow 0} t^{N / 2} \int_{\Omega} k_{t}^{0}(x, x) \mathrm{d} x=\frac{|\Omega|}{(4 \pi)^{N / 2}}
$$

This was the missing inequality required to prove (1.127). Since (1.127) has been shown to be equivalent to Weyl's law, we have completed the proof.

Weyl's law also holds for arbitrary bounded open sets, [137, Theorem 1.11]. A simple proof by approximating an arbitrary open set by regular sets from the interior is given in [138, Section 6.5.2]. For further results on domain approximation we refer to the survey article [139] by Daners.

The proof given here is essentially the one given by Kac [9] who found formula (1.126) and used Karamata's Tauberian theorem. We were also inspired by Dodzink [140] and the Diploma thesis by E. Michel [141]. However, the use of Dirichlet regularity and Theorem 1.2 in particular comes from [138, Chapter 5] where more details can be found. Concerning the Dirichlet problem we refer to $[142,143]$ and the literature mentioned there.

\section{7}

\section{Can One Hear the Shape of a Drum?}

Weyl's law shows us in particular the following. Assume that $\Omega \subset \mathbb{R}^{N}$ is a bounded open set and we know all the eigenvalues of the Dirichlet Laplacian. Then we also know the volume of $\Omega$. Thus the spectrum of the Dirichlet Laplacian determines the volume. It is natural to ask whether there are other properties or qualities which we may deduce from the spectrum. Those types of questions are called inverse (spectral) problems. Let us say that two open bounded sets $\Omega_{1}$ and $\Omega_{2}$ in $\mathbb{R}^{N}$ are isospectral if the corresponding Dirichlet Laplacians $\Delta_{\Omega_{1}}^{\mathrm{D}}$ and $\Delta_{\Omega_{2}}^{\mathrm{D}}$ have the same sequence of eigenvalues. We already know that isospectral sets have the same volume. There is another result of this kind. 
Theorem 1.3 Let $\Omega_{1}, \Omega_{2} \subset \mathbb{R}^{N}$ be open bounded sets with Lipschitz boundary. If $\Omega_{1}$ and $\Omega_{2}$ are isospectral, then they have the same surface area.

Here we use the natural measure $\sigma$ on the boundary $\partial \Omega_{i}$ of $\Omega_{i}$ i. e. the surface measure or (which is the same) the $(N-1)$-dimensional Hausdorff measure. The surface area of $\Omega_{i}$ is by definition $\sigma\left(\partial \Omega_{i}\right)$. For a proof, we refer to [144].

The most radical inverse spectral problem is whether the spectrum determines the domain completely. This question became famous by Marc Kac's article [9] from 1966. We want to formulate it more precisely. Two open sets $\Omega_{1}, \Omega_{2} \subset \mathbb{R}^{N}$ are called congruent if there exists an orthogonal matrix $B$ and a vector $b$ in $\mathbb{R}^{N}$ such that $\Omega_{2}=\left\{B x+b: x \in \Omega_{1}\right\}$. This is just congruence in the Euclidean sense. It is obvious that congruent open sets are isospectral.

Question 1.1 (Kac's Question) Let $\Omega_{1}, \Omega_{2} \subset \mathbb{R}^{2}$ be two bounded smooth domains which are isospectral. Are they necessarily congruent?

By a domain we mean an open connected set. An open bounded set is called smooth if the boundary is of class $\mathrm{C}^{\infty}$.

Kac's question became so popular because it has a fascinating physical interpretation. We consider a bounded smooth domain $\Omega \subset \mathbb{R}^{2}$ as a membrane which is fixed at the boundary $\Gamma$ of $\Omega$. If it is set into motion, then the vertical displacement $u(t, x)$ at time $t>0$ at the point $x \in \Omega$ satisfies the wave equation

$$
u_{t t}=c \Delta u(t, x) \quad(t>0, x \in \Omega) .
$$

We normalize physical units in such a way that $c=1$.

Of particular interest are solutions of the form $u(t, x)=v(x) \mathrm{e}^{i \omega t}$ which are called the pure tones of the membrane. In order that such $u$ be a solution of the wave equation it is necessary and sufficient that

$$
-\Delta v=\omega^{2} v
$$

Thus $u$ is a solution if and only if $v$ is an eigenfunction of the Dirichlet Laplacian for the eigenvalue $\omega^{2}$, where $\omega$ is the frequency of the displacement $u$. Now we see that the eigenvalues of the Dirichlet Laplacian correspond exactly to the pure tones of the membrane which we can hear. This lead Kac to reformulate his question by asking "Can one hear the shape of a drum?". Following Kac, people like to formulate inverse spectral problems by asking which properties of $\Omega$ one can hear. For example, we already know that we can hear the volume and the surface area of a Lipschitz domain.

Kac himself said in [9]: "I believe that one cannot hear the shape of a tambourine but I may be wrong and I am not prepared to bet large sums either way."

Today the question raised by Kac is still open. But much more is known about it. In fact, we may ask more generally if two bounded isospectral domains in $\mathbb{R}^{N}$ are congruent. That is, we consider arbitrary dimensions now and give up the very restrictive smoothness hypothesis. Let us note though that some hypothesis on the 
boundary is needed to avoid trivialities. For instance, if we consider the disc $\Omega_{1}=$ $\left\{x \in \mathbb{R}^{2}:|x|<1\right\}$ and the punctured disc $\Omega_{2}=\Omega_{1} \backslash\{0\}$, then they are isospectral but not congruent. In fact, $L^{2}\left(\Omega_{1}\right)=L^{2}\left(\Omega_{2}\right)$ and also the Dirichlet Laplacians with respect to these two open sets are identical. We will describe below precisely which regularity of the boundary is needed to avoid such simple counterexamples. Here we want to impose throughout that all bounded domains have a Lipschitz boundary, and we call them Lipschitz domains for short. They include all polygons in particular.

Before we describe some of the results concerning Kac's question we mention that the analogous question for compact manifolds has a negative answer as John Milnor [70] had already shown in 1964. So the challenge concerns the Euclidean case. A first counterexample was given by Urakawa [145] in 1982 who constructed two isospectral Lipschitz domains in $\mathbb{R}^{4}$ which are not congruent. Ten years later, Gordon, Webb and Wolpert [146] found a two-dimensional example. By putting together seven triangles they obtained two polygons in $\mathbb{R}^{2}$ which are isospectral but not congruent, see Figure 1.5. These two polygons are not convex, though. It is an open question whether convex isospectral polygons in $\mathbb{R}^{2}$ are congruent. However, in four dimensions convexity alone does not help. There are convex isospectal sets which are not congruent. In fact, by modifying Urakawa's example, Gordon and Webb [147] obtained two truncated convex cones in $\mathbb{R}^{4}$ which are isospectral but not congruent. These cones are induced by some vector space bases in $\mathbb{R}^{4}$. Here is an explicit formulation.

Example 1.2 (Gordon, Webb) Let

$$
u_{1}:=\left(\begin{array}{l}
0 \\
0 \\
1 \\
0
\end{array}\right), u_{2}:=\left(\begin{array}{c}
1 \\
-1 \\
0 \\
0
\end{array}\right), u_{3}:=\left(\begin{array}{l}
1 \\
1 \\
1 \\
0
\end{array}\right), u_{4}:=\left(\begin{array}{l}
0 \\
0 \\
0 \\
1
\end{array}\right)
$$

be the first basis of $\mathbb{R}^{4}$ and

$$
v_{1}:=\left(\begin{array}{l}
1 \\
0 \\
0 \\
0
\end{array}\right), v_{2}:=\left(\begin{array}{c}
1 \\
\sqrt{3} \\
0 \\
0
\end{array}\right), v_{3}:=\left(\begin{array}{l}
0 \\
0 \\
1 \\
0
\end{array}\right), v_{4}:=\left(\begin{array}{l}
0 \\
0 \\
1 \\
1
\end{array}\right)
$$
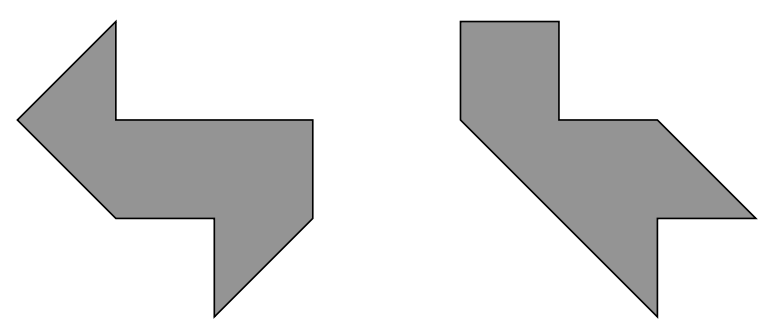

Figure 1.5 Isospectral polygons in two dimensions. 
the second. Consider the corresponding positive cones

$$
C_{1}:=\left\{\sum_{i=1}^{4} a_{i} u_{i}: a_{i} \geq 0, i=1, \ldots, 4\right\}, \quad C_{2}:=\left\{\sum_{i=1}^{4} a_{i} v_{i}: a_{i} \geq 0, i=1, \ldots, 4\right\} .
$$

Let $B_{0}:=\left\{x \in \mathbb{R}^{4}: 0<|x|<1\right\}$ be the punctured unit ball in $\mathbb{R}^{4}$ with respect to the Euclidean norm $|x|=\sqrt{\sum_{j=1}^{4} x_{j}^{2}}$. Then $\Omega_{1}:=B_{0} \cap C_{1}$ and $\Omega_{2}:=B_{0} \cap C_{2}$ are isospectral but not congruent.

So far no smooth counterexample is known in any dimension. But in a very recent work Zelditch [148] showed that isospectral domains with an analytic boundary, having some symmetry, are congruent. A simple class of domains having such a symmetry are ellipses and stadiums. Thus he shows in particular that those domains can be distinguished by their spectra.

Now we describe further positive results. We mention that two isospectral triangles are congruent, see [149] and references therein. Moreover, one can hear whether a Lipschitz domain in $\mathbb{R}^{N}$ is a ball.

Theorem 1.4 Let $\Omega_{1} \subset \mathbb{R}^{N}$ be a ball and $\Omega_{2} \subset \mathbb{R}^{N}$ a Lipschitz domain. If $\Omega_{1}$ and $\Omega_{2}$ are isospectral, then they are congruent.

Proof If $\Omega$ is a Lipschitz domain, then one can hear its volume $|\Omega|$ according to Weyl's law. The Faber-Krahn inequality

$$
\lambda_{1}^{\Omega} \geq c_{N}|\Omega|^{-2 / N}
$$

holds for all such domains, where $\lambda_{1}^{\Omega}$ denotes the first eigenvalue of the Dirichlet Laplacian on $\Omega$ and $c_{N}$ is an optimal constant which depends only on the dimension $N$ [150, Theorem 3.1]. Moreover, (1.130) is an equality if and only if $\Omega$ is a ball, see [151, Theorem 1.2].

The above theorem can be found in Kac's paper [9]. However, Kac uses the isoperimetric inequality together with Theorem 1.3 instead of (1.130). For this argument one has to be able to define the surface area of the domain. The above proof on the other hand works in much more generality. The result can even be made optimal in a sense that we will describe now. For this, we need the notion of capacity which is used to describe the size of sets in $\mathbb{R}^{N}$ in terms of Sobolev norms. For a systematic introduction we refer to [152]. The capacity cap $(A)$ of a set $A \subset \mathbb{R}^{N}$ may be any number in $[0, \infty]$, but here we only need to know whether a set has capacity 0 . Sets of capacity 0 are also called polar sets. Although it is not trivial to characterize all polar sets, thinking of them as subsets of $\mathbb{R}^{N}$ of dimension at most $N-2$ gives a good impression of how they look. For example, single points in $\mathbb{R}^{2}$ and smooth curves in $\mathbb{R}^{3}$ are polar, but curves in $\mathbb{R}^{2}$ and surfaces in $\mathbb{R}^{3}$ are not polar. Moreover, subsets of polar sets and countable unions of polar sets are also polar. 
What makes the notion of capacity paticularly interesting is the fact that the Dirichlet Laplacian "does not see" polar sets. More precisely, if $\Omega_{1}$ and $\Omega_{2}$ are open subsets of $\mathbb{R}^{N}$ that only differ by a polar set i.e. $\Omega_{2} \backslash \Omega_{1}$ and $\Omega_{1} \backslash \Omega_{2}$ are both polar, then the sets differ only by a set of Lebesgue measure zero, hence $L^{2}\left(\Omega_{1}\right)=L^{2}\left(\Omega_{2}\right)$ as subspaces of $L^{2}\left(\mathbb{R}^{N}\right)$. But in fact, $\Delta_{\mathrm{D}}^{\Omega_{1}}=\Delta_{\mathrm{D}}^{\Omega_{2}}$ as operators on this space, thus they have the same spectrum. This shows that inverse spectral problems for the Dirichlet Laplacian are meaningful only up to polar sets. Thus we are lead to introduce a notion of regularity which asserts that there are no artificial "polar holes" in the set. More precisely, call an open set $\Omega$ in $\mathbb{R}^{N}$ regular in capacity if $\operatorname{cap}(B(z, r) \backslash \Omega)>0$ for all $z \in \partial \Omega$ and all $r>0$, where $B(z, r)$ denotes the ball of radius $r$ centered in $z$. We refer to [153] where this regularity assumption is introduced and discussed. Here we only mention that all Dirichlet regular sets, and hence all Lipschitz domains, are regular in capacity.

Given any open set $\Omega \subset \mathbb{R}^{N}$, there exists a unique open set $\Omega^{\prime}$ which is regular in capacity such that $\Omega \subset \Omega^{\prime}$ and $\operatorname{cap}\left(\Omega^{\prime} \backslash \Omega\right)=0$. Since the Laplacian does not see polar sets it is natural to consider merely open sets which are regular in capacity. An inspection of Daners' proof [151] shows that for a bounded open set $\Omega$ which is regular in capacity the Faber-Krahn inequality becomes an identity if and only if $\Omega$ is a ball. Thus Theorem 1.4 remains true if we assume that $\Omega_{2}$ is regular in capacity instead of being a Lipschitz domain. In other words, if $\Omega_{2}$ is an arbitrary open set which is isospectral to a ball $\Omega_{1}$, then the regular version of $\Omega_{2}$ is a ball of the same radius, or, what is the same, there exists a ball $B \subset \mathbb{R}^{N}$ which is a translation of $\Omega_{1}$ such that $\Omega_{2} \subset B$ and $\operatorname{cap}\left(B \backslash \Omega_{2}\right)=0$.

\section{8}

\section{Does Diffusion Determine the Domain?}

In this short section we follow a paradigm which is slightly different from that in the last section. Instead of the wave equation let us consider the diffusion equation

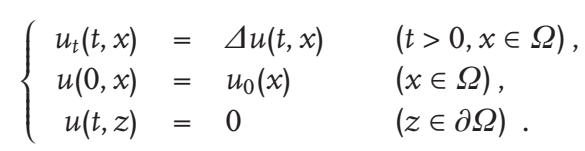

Here again $\Omega$ is a Lipschitz domain with boundary $\Gamma$. The solution $u$ of (D) has the following interpretation. Assume that $\Omega$ is a body containing water and some dissolving liquid, for instance ink. Then $u_{0}$ is the initial concentration of the ink i. e. for $\omega \subset \Omega$ the amount of ink in $\omega$ is given by $\int_{\omega} u_{0}(x) \mathrm{d} x$. The solution $u(t, x)$ gives the concentration at time $t>0$ i. e. for $\omega \subset \Omega, \int_{\omega} u(t, x) \mathrm{d} x$ is the amount of ink in $\omega$ at time $t$.

Given $u_{0} \in L^{2}(\Omega)$, Equation (D) has a unique solution $u: \mathbb{R}_{+} \rightarrow L^{2}(\Omega)$, where we let $u(t, x)=u(t)(x)$, given by

$$
u(t)=\mathrm{e}^{t \Delta_{\Omega}^{\mathrm{D}}} u_{0}=\sum_{n \in \mathbb{N}} \mathrm{e}^{-\lambda_{n} t}\left(u_{0} \mid e_{n}\right) e_{n},
$$


(compare (1.120)). In fact, since $(\mathrm{d} / \mathrm{d} t) \mathrm{e}^{-\lambda_{n} t} e_{n}=\mathrm{e}^{-\lambda_{n} t}\left(-\lambda_{n} e_{n}\right)=\mathrm{e}^{-\lambda_{n} t} \Delta e_{n}, u$ is a solution of (D). Its uniqueness follows from Theorem 1.128, the parabolic maximum principle. Thus the semigroup generated by $\Delta_{\Omega}^{\mathrm{D}}, \mathrm{e}^{-t \Delta_{\Omega}^{\mathrm{D}}}$, is frequently called the diffusion semigroup.

Now let $\Omega_{1}$ and $\Omega_{2}$ be two Lipschitz domains. If $\Omega_{1}$ and $\Omega_{2}$ are isospectral, then we find orthonormal bases $\left(e_{n}\right)_{n \in \mathbb{N}}$ of $L^{2}\left(\Omega_{1}\right)$ and $\left(f_{n}\right)_{n \in \mathbb{N}}$ of $L^{2}\left(\Omega_{2}\right)$ such that

$$
-\Delta_{\Omega_{1}}^{\mathrm{D}} e_{n}=\lambda_{n} e_{n} \quad \text { and } \quad-\Delta_{\Omega_{2}}^{\mathrm{D}} f_{n}=\lambda_{n} f_{n}
$$

for all $n \in \mathbb{N}$. Consider the unitary operator $U: L^{2}\left(\Omega_{1}\right) \rightarrow L^{2}\left(\Omega_{2}\right)$ satisfying $U e_{n}=$ $f_{n}$. Then

$$
U \mathrm{e}^{t \Delta_{\Omega_{1}}^{\mathrm{D}}}=\mathrm{e}^{t \Delta_{\Omega_{2}}^{\mathrm{D}} U} \quad(t>0)
$$

i. e. $U$ intertwines the two diffusion semigroups. In other words, $U$ maps solutions of the first diffusion equation to solutions of the other diffusion equation. Conversely, if we find an intertwining invertible operator $U: L^{2}\left(\Omega_{1}\right) \rightarrow L^{2}\left(\Omega_{2}\right)$, then $\Omega_{1}$ and $\Omega_{2}$ are isospectral. Now we remember that for the physical interpretation only positive concentrations $0 \leq u_{0} \in L^{2}\left(\Omega_{1}\right)$ are meaningful. If $u_{0}(x) \geq 0$ for all $x \in \Omega_{1}$, then $u(t, x) \geq 0$ for all $x \in \Omega_{1}$ and all $t>0$. This is the positivity property of the diffusion equation. The physical interpretation motivates us to consider, instead of unitary operators, operators $U$ which preserve positivity. A linear bijective mapping $U: L^{2}\left(\Omega_{1}\right) \rightarrow L^{2}\left(\Omega_{2}\right)$ is called an order isomorphism if for all $f \in L^{2}\left(\Omega_{1}\right)$, $f \geq 0$ if and only if $U f \geq 0$. If in (1.131) instead of unitary we assume that $U$ is an order isomorphism, then we obtain a positive result.

Theorem 1.5 Let $\Omega_{1}$ and $\Omega_{2}$ be two Lipschitz domains in $\mathbb{R}^{N}$. Assume that there exists an order isomorphism $U: L^{2}\left(\Omega_{1}\right) \rightarrow L^{2}\left(\Omega_{2}\right)$ such that (1.131) holds. Then $\Omega_{1}$ and $\Omega_{2}$ are congruent.

For a proof, we refer to [153, Corollary 3.17]. We remark that this result also remains true if we only assume the domains to be regular in capacity.

This theorem is no longer a purely spectral problem, but it is an inverse problem. To say that $U$ is an intertwining order isomorphism is the same as saying that $U$ maps positive solutions to positive solutions. Thus we may rephrase the result by saying that "Diffusion determines the domain".

\section{References}

1 WeYL, H. (1911) Über die asymptotische Verteilung der Eigenwerte. Nachrichten der Königlichen Gesellschaft der Wissenschaften zu Göttingen. Mathem.physikal. Klasse, 110-117.
2 WeYL, H. (1912) Das asymptotische Verteilungsgesetz der Eigenwerte linearer partieller Differentialgleichungen (mit einer Anwendung auf die Theorie der Hohlraumstrahlung). Mathematische Annalen, 71(4), 441-479. 
3 WeYL, H. (1912) Über die Abhängigkeit der Eigenschwingungen einer Membran von deren Begrenzung. J. Reine Angew. Math., 141, 1-11.

4 Weyl, H. (1912) Über das Spektrum der Hohlraumstrahlung. J. Reine Angew. Math., 141, 163-181.

5 WeYL, H. (1913) Über die Randwertaufgabe der Strahlungstheorie und asymptotische Spektralgeometrie. J. Reine Angew. Math., 143, 177-202.

6 IVRII, V.Y. (1980) Second term of the spectral asymptotic expansion of the Laplace-Beltrami operator on manifolds with boundary. Functional Analysis and Its Applications, 14(2), 98-106.

7 Melrose, R. (1980) Weyl's conjecture for manifolds with concave boundary. Proc. Sympos. Pure Math., 36, 257-274.

8 WeYl, H. (1915) Das asymptotische Verteilungsgesetz der Eigenschwingungen eines beliebig gestalteten elastischen Körpers. Rend. Circ. Mat. Palermo, 39, $1-50$.

9 KAC, M. (1966) Can One Hear the Shape of a Drum? The American Mathematical Monthly, 73(4), 1-23.

10 Sommerfeld, A. (1910) Die Greensche Funktion der Schwingungsgleichung für ein beliebiges Gebiet. Physikal. Zeitschr., 11, 1057-1066.

11 Lorentz, H.A. (1910) Alte und neue Fragen der Physik. Physikal. Zeitschr., 11, 1234-1257.

12 Born, M., Einstein, A. (1972) Briefwechsel 1916-1955. Rowohlt Verlag, 53-55.

13 Reudler, J. (1912) PhD thesis, Leiden.

14 WeYL, H. (1950) Ramifications, old and new, of the eigenvalue problem. Bull. Amer. Math. Soc., 56(2), 115-139.

15 PaIs, A. (1982) "Subtle is the Lord ...", The Science and the Life of Albert Einstein. Oxford University Press.
16 Kirchhoff, G. (1860) Ueber das Verhältniss zwischen dem Emissionsvermögen und dem Absorptionsvermögen der Körper für Wärme und Licht. Annalen der Physik und Chemie, 19, 275301.

17 Hilbert, D. (1912) Begündung der elementaren Strahlungstheorie. Physik. Zeitschrift, 13:1056-1064.

18 Hilbert, D. (1913) Bemerkung zur Begründung der elementaren Strahlungstheorie. Physik. Zeitschrift, 14, 592-595.

19 Hilbert, D. (1914) Zur Begründung der elementaren Strahlungstheorie. Dritte Mitteilung. Physik. Zeitschrift, 15, 878889.

20 Boltzmann, L. (1884) Ableitung des Stefan'schen Gesetzes, betreffend die Abhängigkeit der Wärmestrahlung von der Temperatur aus der electromagnetischen Lichttheorie. Annalen der Physik und Chemie, 22, 291-294.

21 Wien, W. (1893) Eine neue Beziehung der Strahlung schwarzer Körper zum zweiten Hauptsatz der Wärmetheorie. Sitzungsberichte der Königlichen Preußischen Akademie der Wissenschaften zu Berlin, 55-62.

22 Paschen, F. (1896) Ueber Gesetzmässigkeiten in den Spectren fester Körper. (Erste Mittheilung). Annalen der Physik und Chemie, 58, 455-492.

23 Einstein, A. (1913) Max Planck als Forscher. Naturwissenschaften, 1(45), 1077-1079.

24 Wien, W. (1896) Ueber die Energievertheilung im Emissionsspectrum eines schwarzen Körpers. Annalen der Physik und Chemie, 58, 662-669.

25 Paschen, F. (1897) Ueber Gesetzmässigkeiten in den Spectren fester Körper. (Zweite Mittheilung). Annalen der Physik und Chemie, 60, 662-723.

26 Rayleigh, L. (1900) Remarks upon the law of complete radiation. Phil. Mag., 49, 539-540. 
27 Rayleigh, L. (1896) The Theory of Sound, volume 2. Reprinted by Dover Publications, New York, 1945. First edition printed 1877; second revised and enlarged edition.

28 Lummer, O. And Pringsheim, E. (1900) Über die Strahlung des schwarzen Körpers für lange Wellen. Verhandlungen der Deutschen Physikalischen Gesellschaft, 2 , 163-180.

29 Rubens, H. and Kurlbaum, F. (1900) Über die Emission langwelliger Wärmestrahlen durch den schwarzen Körper bei verschiedenen Temperaturen. Sitzungsberichte der Preußischen Akademie der Wissenschaften, 929-941.

30 Planck, M. (1900) Über eine Verbesserung der Wienschen Spektralgleichung. Verhandlungen der Deutschen Physikalischen Gesellschaft, 2, 202-204.

31 WeYL, H. (1931) The theory of groups and quantum mechanics. Dover Publications, New York. Translation of the second (revised) German Edition.

32 Planck, M. (1966) Theorie der Wärmestrahlung. Johann Ambrosius Barth, Leipzig, 6th edition.

33 Planck, M. (1900) Zur Theorie des Gesetzes der Energieverteilung im Normalspectrum. Verhandlungen der Deutschen Physikalischen Gesellschaft, 2, 237-245.

34 Planck, M. (1901) Ueber das Gesetz der Energieverteilung im Normalspectrum. Annalen der Physik, 4, 553-563.

35 Lorentz, H.A. (1903) On the emission and absorbtion by metals of rays of heat of great wave-lengths. Proc. Acad. Amsterdam, 5, 666-685.

36 Einstein, A. (1905) Über einen die Erzeugung und Verwandlung des Lichtes betreffenden heuristischen Gesichtspunkt. Annalen der Physik, 17, 132-148.

37 Rayleigh, L. (1905) The dynamical theory of gases and of radiation. Nature, 72 , $54-55$.
38 Rayleigh, L. (1905) The constant of radiation as calculated from molecular data. Nature, 72, 243-244.

39 Jeans, J.H. (1905) The dynamical theory of gases and of radiation. Nature, 72 , 101-102.

40 Jeans, J.H. (1905) On the partition of energy between matter and aether. Phil. Mag., 10, 91-98.

41 Jeans, J.H. (1905) A comparison between two theories of radiation. Nature, 72, 293-294.

42 Jeans, J.H. (1905) On the laws of radiation. Proc. R. Soc. London A, 76(513), 545-552.

43 Einstein, A. (1949) Albert Einstein: Philosopher-Scientist, (ed P.A. Schilpp), Cambridge University Press, London, 43.

44 Jeans, J.H. (1905) The dynamical theory of gases. Nature, 71:607.

45 Selberg, A. (1956) Harmonic analysis and discontinuous groups in weakly symmetric Riemannian spaces with applications to Dirichlet series. J. Indian Math. Soc., 20, 47-87.

46 Selberg, A. (1962) Discontinuous groups and harmonic analysis, in Proc. Int. Math. Congr. Stockholm, 177-189.

47 Steiner, F. (1987) On Selberg's zeta function for compact Riemann surfaces. Phys. Lett. B, 188, 447-454.

48 Gauss, C.F. (1981) De nexu inter multitudinem classium, in quas formae binariae secundi gradus distribuntur, earumque determinantem, in Werke, 2, 269-280. Georg Olms Verlag, Hildesheim, New York.

49 Hardy, G.H. and Wright, E.M. (2002) An Introduction to the Theory of Numbers, Clarendon Press, Oxford.

50 Hilbert, D. And Cohn-Vossen, S. (1952) Geometry and the Imagination (Anschauliche Geometrie), Chelsea Publishing Company. 
51 LANDAU, E. (1927) Vorlesungen über Zahlentheorie, volume 2, Hirzel Verlag, Leipzig.

52 Sierpiński, W.M. (1974) Oevres Choisies, volume 1, Polish Scientific Publishers PWN, Warsaw.

53 HARDY, G.H. (1915) The average order of the arithmetical functions $P(x)$ and $\Delta(x)$. Proc. London Math. Soc., 15, 192-213.

54 Hardy, G.H.(1915) On the expression of a number as the sum of two squares. Quart. J. of Math., 46, 263-283.

55 van der Corput, J.G. (1923) Neue zahlentheoretische Abschätzungen, erste Mitteilung. Math. Annalen, 89, 215-254.

56 YIN, W.-L. (1962) The lattice points in a circle. Sci. Sinica., 11, 10-15.

57 Iwaniec, H. ANd Mozzochi, C.J. (1988) On the divisor and circle problems. $J$. Number Theory, 29, 60-93.

58 Huxley, M.N. (1993) Exponetial sums and lattice points II. Proc. London Math. Soc., 66, 279-301.

59 Cappell, S.E. And Shaneson, J.L. (2007) Some problems in number theory I: The circle problem. arXiv:math/0702613v3 [math.NT].

60 Hardy, G.H. and Landau, E. (1925) The lattice points of a circle. Proc. Roy. Soc London A, 105, 244-258.

61 Hardy, G.H. (1925) The lattice points of a circle. Proc. Roy. Soc London A, 107, 623-635.

62 Voronoï, G. (1904) Sur le devéloppement, à l'aide des fonctions cylindriques, des sommes doubles $\sum f\left(p m^{2}+2 q m n+r n^{2}\right)$. Verh. Math. Kongr. Heidelberg, 241-245.

63 Steiner, F. (2003) Space-time approach to quantum chaos. physica status solidi (b), 237, 133-145.
64 Aurich, R., Sieber, M. and SteinER, F. (1988) Quantum chaos of the Hadamard-Gutzwiller model. Phys. Rev. Lett., 61(5), 483-487.

65 Aurich, R. and Steiner, F. (1989) Periodic-orbit sum rules for the Hadamard-Gutzwiller model. Physica D, 39, 169-193.

66 Aurich, R. and Steiner, F. (1992) From classical periodic orbits to the quantization of chaos. Proc. R. Soc. Lond. A, 437, 693-714.

67 Bolte, J. and Steiner, F. (1990) Determinants of Laplace-like operators on Riemann surfaces. Commun. Math. Phys., 130, 581-597.

68 Bolte, J. and Steiner, F. (1993) The Selberg trace formula for bordered Riemann surfaces. Commun. Math. Phys., 156, 1-16.

69 Aurich, R., Janzer, H.S., Lustig, S. And Steiner, F. (2008) Do we live in a "small Universe"? Classical and Quantum Gravity, 25, 125006, 1-12.

70 Milnor, J. (1964) Eigenvalues of the Laplace Operator on Certain Manifolds. Proc. Nat. Acad. Sci. USA, 51(4), $542 \mathrm{ff.}$

71 WitT, E. (1941) Eine Identität zwischen Modulformen zweiten Grades. Abh. Math. Sem. Hansischen Universität, 14, 323-337.

72 Chazarain, J. (1974) Formule de poisson pour les variétés riemanniennes. Invent. Math., 24, 65-82.

73 Colin de Verdière, Y. (1973) Spectre du laplacien et longueurs des géodésiques périodiques. i. Compos. Math., 27(1), 83-106.

74 Colin de Verdière, Y. (1973) Spectre du laplacien et longueurs des géodésiques périodiques. ii. Compos. Math., 27(2), 159-184.

75 DuistermaAt, J.J. and Guillemin, V.W. (1975) The spectrum of positive elliptic operators and periodic bicharacteristics. Invent. Math., 29, 39-79. 
76 Guillemin, V.W. (1977) Lectures on spectral theory of elliptic operators. Duke Math. J., 44, 485-517.

77 Minakshisundaram, S. and Pleijel, A. (1949) Some properties of the eigenfunctions of the Laplace-operator on Riemannian manifolds. Can. J. Math., 1, 242-256.

78 MinaKshisundaram, S. (1949) A generalization of Epstein zeta function. Can. J. Math., 1, 320-329.

79 Titchmarsh, E.C. (1948) Introduction to the theory of Fourier integrals. Oxford University Press, 2nd edition.

80 Ray, D. And Singer, I.M. (1973) Analytic torsion for complex manifolds. Ann. Math., 98, 154-177.

81 Hawking, S. (1977) Zeta function regularization of path integrals in curved space time. Commun. Math. Phys., 55, 133-148.

82 Grosche, C. And Steiner, F. (1998) Handbook of Feynman path integrals, volume 145 of Springer Tracts in Modern Physics. Springer.

83 Hughes, C.P. And Rudnick, Z. (2004) On the distribution of lattice points in thin annuli. Int. Math. Research Notices, 13, 637-658.

84 Cramér, H. (1922) Über zwei Sätze von Herrn G.H. Hardy. Math. Z., 15, 201-210.

85 Heath-Brown, D.R. (1992) The distribution and moments of the error term in the Dirichlet divisor problem. Acta Arithmetica, LX(4):389-415.

86 Bleher, P.M., Cheng, Z., Dyson, F.J. AND LebowitZ, J.L. (1993) Distribution of the error term for the number of lattice points inside a shifted circle. Communications in Mathematical Physics, 154(3), 433-469.

87 Levitan, B.M. and Zhikov, V.V. (1968) Almost Periodic Functions and Differential Equations. Cambridge University Press.
88 Aurich, R., BÄCKer, A. And Steiner, F. (1997) Mode fluctuations as fingerprints of chaotic and non-chaotic systems. Int. J. Modern Phys. B, 11, 805-849.

89 Safarov, Y. and Vassiliev, D. (1996) The Asymptotic Distribution of Eigenvalues of Partial Differential Operators, volume 155 of Translations of Mathematical Monographs. AMS.

90 Brownell, F.H. (1957) Extended asymptotic eigenvalue distributions for bounded domains in $n$-space. J. Math. Mech., 6, 119-166.

91 Baltes, H.P. And Hilf, E.R. (1976) Spectra of Finite Systems, Bibliographisches Institut Mannheim, Zürich.

92 Steiner, F. (1994) Quantum chaos. In R. Ansorge, editor, Schlaglichter der Forschung. Zum 75. Jahrestag der Universität Hamburg 1994, Dietrich Reimer Verlag, Berlin, 543-564.

93 Aurich, R., Bolte, J. and Steiner, F. (1994) Universal signatures of quantum chaos. Phys. Rev. Lett., 73, 1356-1359.

94 Berry, M.V. (1985) Semiclassical theory of spectral rigidity. Proc. R. Soc. London, A 400, 229-251.

95 Bleher, P.M. ANd Lebowitz, J.L. (1994) Energy-level statistics of model quantum systems: Universality and scaling in a lattice-point problem. J. Statistical Physics, 74, 167-217.

96 Bleher, P.M. (1999) Trace formula for quantum integrable systems, latticepoint problem, and small divisors, in (eds D.A. Hejhal, J. Friedman, M.C. Gutzwiller and A.M. Odlyzko), Emerging Applications of Number theory, Springer, New York, 1-38.

97 Aurich, R. and Steiner, F. (1993) Statistical properties of highly excited quantum eigenstates of a strongly chaotic system. Physica D, 64, 185-214.

98 Selberg, A. (1946) Contributions to the theory of the Riemann zeta-function. Arch. Math. Naturvid., 48(5), 89-155. 
99 GHosh, A. (1983) On the Riemann zetafunction - mean value theorems and the distribution of $\mathrm{S}(\mathrm{T})$. J. Number Theo., 17(1), 93-102.

100 Montgomery, H.L. (1989) Selberg's work on the zeta-function, in (eds K.E. Aubert, E. Bombieri and D. Goldfeld), Number Theory, Trace Formulas and Discrete Groups, Academic, New York, 157168.

101 BÄCKer, A. ANd Steiner, F. (2001) Quantum chaos and quantum ergodicity, in (ed B. Fiedler), Ergodic Theo$r y$, Analysis, and Efficient Simulation of Dynamical Systems, Springer, Berlin, 717-751.

102 Carleman, T. (1934) Propriétés asymptotiques des fonctions fondamentales des membranes vibrantes. Comptes Rendus des Mathematiciens Scandinavesa Stockholm, 14-18.

103 Carleman, T. (1936) Über die asymptotische Verteilung der Eigenwerte partieller Differentialgleichungen. Ber. der Sächs. Akad. d. Wiss. Leipzig, 88, 119132.

104 Hadamard, J. (1898) Sur le billard non-Euclidean. Soc. Sci. Bordeaux, Proc. Verbaux, p. 147.

105 HADAMARD, J. (1898) Les surfaces à courbures opposées et leurs lignes géodésiques. J. Math. Pure Appl., 4, 27-73.

106 Gutzwiller, M.C. (1980) Classical quantization of a Hamiltonian with ergodic behavior. Phys. Rev. Lett., 45, 150-153.

107 Aurich, R. And Steiner, F. (2001) Orbit sum rules for the quantum wave functions of the strongly chaotic Hadamard billiard in arbitrary dimensions. Foundations of Physics, 31, 423-444.

108 Aurich, R. And Steiner, F. (2001) The cosmic microwave background for a nearly flat compact hyperbolic universe. Monthly Notices of the Royal Astronomical Society, 323, 1016-1024.
109 Grosche, C. and Steiner, F. (1988) The path integral on the pseudosphere. Annals of Physics (NY), 182, 120-156.

110 Aurich, R. And Steiner, F. (1991) Exact theory for the quantum eigenstates of a strongly chaotic system. Physica D, 48, 445-470.

111 Buser, P. (1977) Riemannsche Flächen mit Eigenwerten in $(0,1 / 4)$. Comment. Math. Helvet., 52(1), 25-34.

112 Schoen, R., Wolpert, S. and Yau, S.T. (1980) Geometric bounds on the low eigenvalues of a compact surface, in $\mathrm{Ge}$ ometry of the Laplace operator, volume 36 of Proc. Sympos. Pure Math., American Mathematical Society, Providence, 279-285.

113 Schmutz, P. (1991) Small eigenvalues on Riemann surfaces of genus 2. Invent. Math., 106(1), 121-138.

114 Titchmarsh, E.C. (1986) The theory of the Riemann zeta-function. Oxford University Press, Second edition revised by D.R. Heath-Brown.

115 Ingham, A.E. (1971) The distribution of prime numbers, Hafner, New York.

116 Weil, A. (1952) Sur les 'formules explicites' de la théorie des nombres premiers. Comm. Sém. Math. Univ. Lund (Medd. Lunds Univ. Mat. Sem) Tome Supplémentaire, 252-265.

117 Connes, A. (1996) Formule de trace en géométrie non-commutative et hypothèse de Riemann. C.R. Acad. Sci. Paris, 323:(1231)-(1236).

118 http://www.dtc.umn.edu/ odlyzko/ polya/index.html.

119 Deitmar, A. (2000) Geometric zetafunctions of locally symmetric spaces. Am. J. Math., 122(5), 887-926.

120 Ruelle, D. (1976) Zeta-functions for expanding maps and Anosov flows. Invent. Math., 34(3), 231-242. 
121 Aurich, R. and Steiner, F. (1992) Asymptotic distribution of the pseudoorbits and the generalized Euler constant $\gamma_{\Delta}$ for a family of strongly chaotic systems. Phys. Rev. A, 46, 771-781.

122 Huber, H. (1959) Zur analytischen Theorie hyperbolischer Raumformen und Bewegungsgruppen. Math. Ann., 138(1), 1-26.

123 Aurich, R. and Steiner, F. (1992) Staircase functions, spectral rigidity, and a rule for quantizing chaos. Phys. Rev. A, 45(2), 583-592.

124 Balian, R. and Bloch, C. (1970) Distribution of eigenfrequencies for the wave equation in a finite domain. I. Threedimensional problem with smooth boundary surface. Ann. Phys., 60(43), 401-447.

125 Balian, R. AND Bloch, C. (1971) Distribution of Eigenfrequencies for the Wave Equation in a Finite Domain. II. Electromagnetic Field. Riemannian Spaces. Ann. Phys., 64(43), 271-307.

126 Balian, R. and Bloch, C. (1974) Errata. Ann. Phys., 84, 559-563.

127 Sieber, M., Primack, H., Smilansky, U., Ussishrin, I. ANd Schanz, H. (1995) Semiclassical quantization of billiards with mixed boundary conditions. J. Phys. A: Math. Gen., 28, 5041-5078.

128 Simon, B. (1983) Some quantum operators with discrete spectrum but classically continuous spectrum. Ann. Phys. (NY), 146(1), 209-220.

129 Simon, B. (1983) Nonclassical eigenvalue asymptotics. J. Functional Analysis, 53(4), 84-98.

130 Steiner, F. And Trillenberg, P. (1990) Refined asymptotic expansion for the partition function of unbounded quantum billiards. J. Math. Phys., 31, 16701676 .

131 Sieber, M. ANd Steiner, F. (1990) Classical and quantum mechanics of a strongly chaotic billiard system. Physica $D, 44,248-266$.
132 Sieber, M. and Steiner, F. (1990) Quantum chaos in the hyperbola billiard. Phys. Lett. A, 148(8-9), 415-420.

133 Sieber, M. and Steiner, F. (1991) Quantization of chaos. Phys. Rev. Lett., 67(15), 1941-1944.

134 Karamata, J. (1931) Neuer Beweis und Verallgemeinerung der Tauberschen Sätze, welche die Laplace'sche und Stieljes Transformation betreffen. J. Reine Angew. Math., 164, 27-39.

135 Arendt, W., Batty, C., Hieber, M. And Neubrander, F. (2001) Vector-Valued Laplace Transforms and Cauchy Problems, Birkhäuser.

136 Arendt, W. (2000) Resolvent positive operators and inhomogeneous boundary conditions. Scuola Normale Superiore Pisa, 29, 639-670.

137 Birman, M.S. ANd SolomjaK, M.Z. (1980) Quantitative Analysis in Sobolev Imbedding Theorems and Applications to Spectral Theory. American Mathematical Society.

138 ArendT, W. (2005/06) Heat Kernels (Internet Seminar 2005/2006). https://tulka.mathematik.uni-ulm.de/ 2005/lectures/internetseminar.pdf.

139 DANERs, D. (2008) Handbook of Differential Equations: Stationary Partial Differential Equations, 6, 1-81, Elesevier.

140 Dodziuk, J. (1981) Eigenvalues of the Laplacian and the heat equation. The American Mathematical Monthly, 88(9), 686-695.

141 Michels, E. (2001) Zur Spektraltheorie elliptischer Differentialoperatoren: Beschreibung und Informationsgehalt diskreter Eigenwerte. Master's thesis, Eberhard-Karls-Universität Tübingen.

142 Arendt, W. ANd Daners, D. (2008) The Dirichlet problem by variational methods. Bulletin of the London Mathematical Society, 40, 51-56. 
143 Arendt, W. ANd Daners, D. (2008) Varying Domains: Stability of the Dirichlet and Poisson Problem. Discrete Continuous Dynamical Systems - Series A, 21, 21-39.

144 Brown, R.M. (1993) The trace of the heat kernel in Lipschitz domains. Transactions of the American Mathematical Society, 339(2), 889-900.

145 Urakawa, H. (1982) Bounded domains which are isospectral but not congruent. Annales Scientifiques de l'École Normale Supérieure Sér. 4, 15(3), 441-456.

146 Gordon, C., WebB, D. And Wolpert, S. (1992) Isospectral plane domains and surfaces via Riemannian orbifolds. Inventiones Mathematicae, 110(1), 1-22.

147 Gordon, C. ANd WebB, D. (1994) Isospectral convex domains in euclidean space. Math. Res. Lett, 1, 539-45.

148 Zelditch, S. (2007) Inverse Spectral Problem for Analytic Domains II: $\mathbb{Z}_{2-}$
Symmetric Domains, Annals of Mathematics, to appear.

149 Chang, P.K. and Deturck, D. (1989) On hearing the shape of a triangle. Proceedings of the American Mathematical Society, 105(4), 1033-1038.

150 Henrot, A. (2003) Minimization problems for eigenvalues of the Laplacian. Journal of Evolution Equations, 3, 443461.

151 Daners, D. ANd Kennedy, J. (2007) Uniqueness in the Faber-Krahn inequality for Robin problems. SIAM J. Math. Anal., 39(4), 1191-1207.

152 Ziemer, W.P. ANd Malỳ, J. (1997) Fine Regularity of Solutions of Elliptic Partial Differential Equations. Am. Math. Soc.

153 Arendt, W. (2002) Does diffusion determine the body? J. Reine. Angew. Math, 550, 97-123. 
